9

10

\title{
Chromatin reprogramming and transcriptional regulation orchestrate embryogenesis in hexaploid wheat
}

\author{
Long Zhao ${ }^{1,2^{*}}$, Xuelei $\operatorname{Lin}^{1 \#}$, Yiman Yang ${ }^{1,4 \#}$, Xiaomin $\mathrm{Bie}^{3}$, Hao Zhang ${ }^{1,2}$, Jinchao \\ Chen $^{1,2}$, Xuemei Liu ${ }^{1,2}$, Hao Wang ${ }^{1,2}$, Jiafu Jiang ${ }^{4}$, Xiangdong $\mathrm{Fu}^{1,2}$, Xiansheng Zhang ${ }^{3}$, \\ Jun Xiao ${ }^{1,2,5^{*}}$ \\ ${ }^{1}$ Key Laboratory of Plant Cell and Chromosome Engineering, Institute of Genetics and \\ Developmental Biology, Chinese Academy of Sciences, Beijing 100101, China \\ ${ }^{2}$ University of Chinese Academy of Sciences, Beijing 100049, China \\ ${ }^{3}$ Shandong Agriculture University, Tai'an, Shandong, China \\ ${ }^{4}$ Nanjing Agriculture University, Nanjing, Jiangsu, China \\ ${ }^{5}$ CAS-JIC Centre of Excellence for Plant and Microbial Science (CEPAMS), Institute \\ of Genetics and Developmental Biology, Chinese Academy of Sciences, Beijing, \\ 100101, China \\ \# These authors contributed equally to this work \\ *Correspondence to zhaolong@genetics.ac.cn; jxiao@genetics.ac.cn
}

\section{ABSTRACT}

Embryogenesis represents the beginning of life cycle, but our understanding of the regulatory circuitry in plants is far lagged to animals. Here, we draw a transcriptome trajectory and chromatin landscape profile during embryogenesis of most cultivated crop hexaploid wheat, highlighting large-scale chromatin reconfiguration and distinct proximal and distal transcriptional regulation in defining cell fate transition. Upon fertilization, $\mathrm{H} 3 \mathrm{~K} 27 \mathrm{ac}$ and $\mathrm{H} 3 \mathrm{~K} 4 \mathrm{me} 3$ resetting were correlated with maternal genome silence, while de novo building of chromatin accessibility activated zygotic genome. Global depletion of $\mathrm{H} 3 \mathrm{~K} 27 \mathrm{me} 3$ in pre-embryo results in a permissive chromatin environment with gain-of-chromatin accessibility, allowing subsequent hierarchical cis- and trans-regulation network mediated by key factors, such as LEC1, MYB, ZHD, LEC2, governing embryo pattern formation. By contrast, H3K27me3 restoration coordinating with chromatin compaction in developmental genes attenuated totipotency and prohibited extensive organogenesis during embryo maturation. In addition, dynamic biased expression of homeolog triads and diverse expression profiles after polyploidization were observed. This is correlated with asymmetric transposon elements insertion in accessible proximal and distal regions. Thus, our study revealed a plant-specific chromatin reprogramming process in facilitating the 
34 hierarchical transcription regulation circuits mediated "inverse hourglass model" and unveiled epigenetic regulation of evolutionary divergence among different sub-genome in shaping embryogenesis in polyploidy wheat.

\section{Introduction}

Embryogenesis, during which fusion of parental terminally differentiated gametes following fertilization generates an entirely new organism, represents the beginning of development and ensures the continuation of the life cycle for both plants and animals ${ }^{1-4}$. Plant embryogenesis can be generally divided into three steps: early-embryogenesis, during which a totipotent zygote was formed following fertilization; mid-embryogenesis, during which the major cell lineages and embryo body pattern was built; and late-embryogenesis, during which nutrition was accumulated in mature embryo followed by seed dormancy ${ }^{1,2}$. Comparisons between animals and plants' embryonic development highlight both conservation and difference. Animals and plants share a similar cell fate determination process-cell division followed by cell identity specification during embryonic development. In addition, both the transcriptional patterns of animals and plants go through the same transcriptomic hourglass model where a conserved phylotypic period during mid-embryogenesis punctuating divergent early- and late-stage between species with a phylum and the inverse hourglass model where gene expression patterns during early- and late-stage are more comparable than mid-stage ${ }^{5,6}$. Nevertheless, there are also differences between the two kingdoms: maternal dominant versus both parental contribution to early-embryogenesis, completed versus partial organogenesis, with dormancy or not, etc ${ }^{1,7,8}$. Besides the morphological and transcriptome similarity and difference, a large gap is the chromatin dynamics comparison between two kingdoms, due to the lack of comprehensive epigenomic study in plants. 
61 cues $^{9,10}$. The technological advances for epigenetics landscape profiling, such as 62 histone modifications, DNA accessibility and DNA methylation, etc., greatly facilitate 63 the discovery of cis-regulatory elements and other non-coding genomic features associated with development, environment, disease and cancer ${ }^{11-13}$. Epigenetic regulatory logic ensures the precise progress and timing of maternal-to-zygotic transition (MZT), zygotic gene activation (ZGA), cell-fate decision, and lineage-specific differentiation during animal embryogenesis ${ }^{14,15}$. A large-scale epigenetic remodeling occurred during embryogenesis, including histone modifications, chromatin accessibility and 3D chromatin organization, as well as DNA methylation ${ }^{4,16}$. H3K4me3 is rapidly depleted after fertilization but 71 re-established during major ZGA and its active removal is important for ZGA 72 initiation ${ }^{17}$. Besides, a noncanonical form of $\mathrm{H} 3 \mathrm{~K} 4 \mathrm{me} 3$ was present in both humans 73 and mice at distal intergenic regions, though the exact function is not clear ${ }^{18}$. Global erasure of H3K27me3 occurred during early embryogenesis, but with parental genomic allelic difference manner ${ }^{4}$. As well, H3K27me3 could be inter-generationally inherited from the maternal genome to regulator enhancer activity during $\mathrm{MZT}^{19}$. Plus, H3K4me3 and H3K27me3 co-occupy lineage-specific genes at the blastocyst stage suggest a critical role of bivalency in poised state maintenance and gene expression regulation ${ }^{20}$. Major chromatin accessibility reorganization is critical for epigenetic reprogramming to convert terminally differentiated gametes into a totipotent state ${ }^{21}$. Open chromatin was progressively established during early embryogenesis and showed a major increase in 8-cell embryos in mouse $\mathrm{e}^{22,23}$, correlated with gene activation. However, previous studies on plant embryogenesis have mainly focused on the transcriptional change and functional identity of specific genes at certain embryo development stages ${ }^{8,24-28}$. A systematic view of how epigenomic landscape contributes to genes regulation in cell fate transition, lineage specification and cellular metabolic accumulation during plant embryogenesis is lacking, especially for crops ${ }^{29}$. 
protein daily consumed and is important for human sustention. The majority of global wheat production comes from two species, the hexaploid bread wheat (Triticum aestivum), which accounts for $95 \%$ of global wheat production and tetraploid pasta wheat (Triticum turgidum var durum). Hexaploid wheat (AABBDD) is the result of two times hybridization and polyploidization events of three putative diploid wild grass progenitor species, Triticum monococcum (AA), an Aegilops speltoides-related species (BB), and Aegilops tauschii (DD) $)^{30,31}$. A recent finding investigated the transcriptional landscape of polyploidy wheat and diploid ancestors during embryogenesis ${ }^{27}$. Through comparison among different ploidy wheat, the evolutionary divergence of gene expression and contribution of A, B, D subgenome to grain development in polyploidy wheat were characterized ${ }^{27}$. Yet, without further information such as epigenomic profiles, it would be hard to dissect the complex transcription regulation network governing such programmed cell fate transition process and elucidation of driving force for the ontogenetic divergence along with the evolution of polyploidy wheat species. Besides, the elucidation of transcriptional complexity and epigenome atlas during embryonic development could be helpful to study in other developmental processes and plant breeding applications, such as parthenogenesis and somatic embryogenesis ${ }^{32,33}$.

Here, we investigate various histone modifications and chromatin accessibility of eight-stage samples across embryonic development of hexaploid wheat, providing a systematic view of transcriptome trajectory and chromatin landscape profiles. Thousands of distal genomic regulatory elements and putative enhancer-gene linkages are identified to drive embryogenesis. A plant-specific large-scale chromatin state reconfiguration and transcriptional regulation circuitry were characterized during embryonic phase transition. Further, we provide evidence for an explanation of the regulatory mechanisms underlining evolutionary divergence among different sub-genomes and the 'inverse hourglass model' of transcription variability during hexaploid wheat embryogenesis. This data thus provides a comprehensive resource of 
117 genetic and epigenomic annotations for researchers studying transcriptional regulation,

118 embryonic development and polyploid evolution.

119 Result

120 Charting the chromatin landscapes during wheat embryogenesis

121 Despite the importance of transcription (re)programming and epigenetic 122 regulation during plant embryogenesis, a comprehensive survey is limited for both 123 model plants and crops ${ }^{29}$. This is partly due to the difficulty to get robust epigenomic 124 data from a limited sample of developing embryos. Here, the Cleavage Under Targets 125 and Tagmentation (CUT\&Tag) and Assay for Transposase Accessible Chromatin with 126 high-throughput sequencing (ATAC-seq) were used to tackle this challenge ${ }^{34,35}$. In a 127 pilot run, CUT\&Tag showed not only high correlation with published Chromatin 128 immunoprecipitation (ChIP)-seq data (correlation $>0.8)$ but also high repeatability and 129 sensitivity with lower nuclei input ( 1,000 nuclei/reaction) and sequencing depth 130 ( 1/3-1/20 of ChIP-seq) (Supplementary Fig. 1a-c). ATAC-seq from about 5,000 nuclei

131 showed logical peak numbers, good signal-to-noise ratio, reasonable fragment size and 132 genomic distribution, and fully meet the demand of transcription factors (TFs) footprint 133 calling (Supplementary Fig. 1d-h).

134 With this capability, the 'reference epigenome' of eight embryonic 135 developmental stages were generated (DPA $0,2,4,6,8,12,16,22$, days post 136 anthesis), including histone modifications, histone variant and RNA Polymerase II 137 profile and chromatin accessibility, as well as transcriptome (Fig. 1a). In general, 138 various chromatin marks showed featured distribution patterns and logical correlation 139 with transcription (Supplementary Fig. 2a, b). As well, pair-wise correlations analysis 140 showed that active markers (H3K27ac, H3K9ac, H3K4me3, Pol II, H3K36me3, 141 H3K4me1, H2A.Z and ATAC) correlate positively with one another but showed 142 varying degrees of exclusivity with repressive markers (H3K27me3, H3K9me2, 
143 H3K9me3) (Supplementary Fig. 2c). Unsupervised PCA and hierarchical clustering of 144 RNA-seq and ATAC-seq revealed a continuous trajectory which can be divided into 145 five major groups roughly corresponding to fertilization, pre-embryo, transition, 146 differentiation and maturation of embryo (Fig. 1b, e). In contrast, core histone 147 modifications showed a phase transition pattern, which revealed a global 148 reconfiguration of chromatin at a certain stage (Supplementary Fig. 2d). GO 149 enrichment of time-lapse correlated modules revealed that cell cycle and DNA 150 replication-related genes were activated during early embryogenesis, organ and tissue 151 specification-related processes were distinguished during mid-development, and 152 nutrient accumulation and dormancy were built during the late stage (Fig. 1c). This is 153 evident in the evolutionary conservation of monocots and dicots embryogenesis ${ }^{27,28}$. 154 In particular, some known factors involved in embryo pattern formation, such as 155 WOX11, LEC2, ARR6 and ABI5, showed similar expression patterns during 156 embryogenesis among wheat, Brachypodium and Arabidopsis but not the other genes, 157 such as YUC1 (Fig. 1d). Furthermore, chromatin accessibility was progressively built 158 until DPA 8, transition stage for embryo patterning, whereas gradually closed during 159 differentiation stage (Fig. 1f). Notably, the majority of ATAC-seq peaks are more than $1603 \mathrm{~Kb}$ even $20 \mathrm{~Kb}$ away from transcriptional start site (TSS) of genes (Fig. 1g), which is 161 different from plants of small genome size $\mathrm{e}^{34,36}$. Interestingly, more ATACseq peaks 162 distributed even further away from TSS in B subgenome as compared to A or D 163 subgenome (Fig. 1g), coincidently with the fact that B subgenome is larger than A or $164 \mathrm{D}^{31}$.

165 Though it is hard to isolate purified embryo at early embryonic development 166 stages (DPA 0,2, 4), the progressive change of transcription and chromatin 167 accessibility presented here indicates a logical and meaningful cellular process. Thus, 168 our 'reference epigenome' could capture the dynamics of transcription and potential 169 transcriptional regulation features during wheat embryogenesis process. 


\section{Distinct feature of proximal and distal chromatin accessible regions} and correlation with transcription regulation

Accessible chromatin can expose regulatory DNA sequences to transcription factors, and thus emerges as an accurate proxy for transcription regulatory potential

174 and development ${ }^{37}$. In this study, we identified 1,315,547 accessible chromatin 175 regions (ACRs) from ATAC-seq in total, which could be further categorized into genic $176(\mathrm{~g})$, promoter (p) and distal (d)ACRs based on the location relative to genes, with a 177 better correlation between dACRs, pACRs and gene expression than gACRs (Fig. 2a).

178 Interestingly, the gain and loss of pACRs and dACRs varied largely during 179 embryogenesis (Fig. 2b and Supplementary Fig. 3a, b). Lots of genes gained pACRs 180 after fertilization at DPA2 but dramatically lost during late embryogenesis at DPA1

181 and DPA16. Whereas dACRs, accounting for about $75 \%$ of total ACRs, showed a 182 sharp 'burst' at DPA8, the transition stage between cell division and differentiation, 183 then quickly declined at DPA12 (Fig. 2b). Genome-wide ACRs distribution showed 184 that centromere region and distal arms of chromosome including telomere are the "hot 185 spot' for chromatin loose-tight regions, with highly overlapped with Gypsy and 186 CACTA types of TEs. In addition, there was a highly synchronized distribution 187 pattern between ACRs and $\mathrm{H} 3 \mathrm{~K} 9 \mathrm{me} 3$, but not other histone modifications, at those 188 chromatin eruptions hot spots (Fig. 2b and Supplementary Fig. 3c). This may be 189 correlated with transient expression of TEs just like the activation of retrotransposons 190 in mouse and human 2-cell stage embryo which is critical for the activation of ZGA 191 genes during early embryogenesis ${ }^{4}$. Besides the temporal dynamics, subgenomes of 192 hexaploid wheat showed slight differences for the chromatin accessibility, especially 193 at centromere and telomere regions in addition to the low-colinear chromosome 194 regions (Fig. 2d and Supplementary Fig. 3d).

195 The different remodeling pattern of pACR and dACR suggests a distinct 196 regulation weight of gene expression during embryogenesis. Indeed, the 
stage-abundant pACRs did not perfectly fit corresponding genes, as well as functional pathway enrichment (Fig. 2e, f and Supplementary Fig. 4a). We thus assigned dACRs to corresponding genes using a correlation strategy as the previous report (Method; Supplementary Fig. 4 b) ${ }^{38}$. In total, we identified 128,212 regulatory dACR (termed reg-dCAR) linked to 43,901 genes, which accounts for $60 \%$ of expressed genes during embryo development and shows higher expression variation (Fig. $2 \mathrm{~g}$ and Supplementary Fig. 4c). High interaction frequency between reg-dACRs and targets measured by $\mathrm{Hi}-\mathrm{C}$ and conservation among various species emphasized the accuracy of this distal assignment strategy (Supplementary Fig. 4d, e). Only 35\% reg-dACRs are assigned to the nearest genes (Supplementary Fig. 4f, g). Most reg-dACRs have only one target, while one gene could be regulated by multiple dACRs (Supplementary Fig. 4h, i). Genes with few reg-dACRs regulations were enriched for essential biological processes, whereas multiple reg-dACR regulated genes are preferred in stress response and organ development (Supplementary Fig. 4j). The assigned target genes of stage-specific reg-dACRs are well associated with specified developmental stages during embryogenesis (Fig. 2g). Of interest, about $60 \%$ of the reg-dACRs were located within TEs of different types (Fig. 2h). Interestingly, the accessibility of reg-dACRs and expression of linked genes were sensitive to TEs classes where they are located (Supplementary Fig. 4k, 1). Besides, reg-ACRs were highly overlapped with H2A.Z, a histone variant with looser DNA wrapping as compared to canonical $\mathrm{H}_{2} \mathrm{~A}^{39}$ (Fig. 2i). Indeed, more than $80 \%$ H2A.Z signal located within reg-ACRs, and reg-dACRs with H2A.Z showed higher 'enhancer activity' with more accessibility and higher expression of targeted genes (Fig. 2i).

Thus, proximal and distal chromatin accessibility undergoes distinct reprogramming patterns during the process of embryogenesis based on genomic features underlying, with different functional output. Meanwhile, a large proportion of long-range distal regulation and asymmetry of chromatin accessibility dynamics among subgenomes exhibit uniqueness for the huge allohexaploid wheat genome. 


\section{Chromatin reprogramming during wheat embryogenesis}

To generally classify chromatin states, we integrated eight histone modification marks and Pol II profiles from four embryonic developmental stages (DPA 0, 2, 4, 8) using ChromHMM ${ }^{40,41}$. Totally 15 chromatin states were categorized, covering $0.02 \%$ to $3.5 \%$ of the genome respectively (exclude No-signal states) (Fig. 3a). They further

230 grouped into five major functional classes: Promoter (Pr), Enhancer-like (EnL),

231 Transcriptional (Tr), Polycomb group (PcG), and Heterochromatin (Hc) (Fig. 3a).

232 Promoter and enhancer-like classes are enriched for active histone modifications such 233 as H3K27/9ac, H3K4me1/3, chromatin accessibility and histone variant H2A.Z (Fig.

234 3a). As expected, chromatin states and histone modifications underlying were 235 dramatically altered across developmental stages, with the enhancer-like class varied 236 the most than promoters, just like the case in mouse fetal development ${ }^{42}$ (Fig. 3b, c). 237 This is correlated with the dramatic transcriptome fluctuation (Fig. 1b, c) and 238 regulatory role of dACRs and pACRs (Fig. 2e, h). In addition, the chromatin state 239 pattern at DPA0 is distinguished from other stages (Fig. 3d), revealing a drastic 240 histone modification reprogramming after fertilization.

241 In mice and humans, histone modifications such as H3K4me3, H3K27me3, and 242 H3K9me3 are extensively reprogrammed after fertilization and pivotal for early 243 embryogenesis ${ }^{4,16}$. Thus, we take a close look at their behaviors in wheat embryonic 244 development. Expect for H3K36me3 and H3K4me1, most of histone modifications 245 examined show a heavy proportion distributed in the distal intergenic regions 246 (Supplementary Fig. 2a). We investigated the dynamic profiles of each histone 247 modification with separation of proximal and distal regions. Different from animals, 248 genome-wide H3K4me3 showed a slight change during embryogenesis of wheat at 249 both proximal and distal regions (Fig. 3e and Supplementary Fig. 5a). Whereas, $250 \mathrm{H} 3 \mathrm{~K} 27 \mathrm{ac}$ presented a quick decline after fertilization, then restore and maintained 251 high till mid-embryo followed by gradually decreasing at late and mature embryo 
252 stages (Fig. 3e and Supplementary Fig. 5a). For H3K27me3, instead of being erased 253 quickly after fertilization like the case in animals, a sharp erasure occurred at 254 pre-embryo stage then gradually restored and kept high at late and maturation stages. 255 H3K9me3 fluctuates slightly after fertilization and during pre-embryo, and then 256 increased through mid-embryo till maturation (Fig. 3e and Supplementary Fig. 5a). 257 Besides the individual histone modification, H3K4me3 and H3K27me3 co-occupied 258 regions are referred as bivalent chromatin, which is correlated with and function in 259 lineage-specific genes activation or silencing during embryonic development in 260 animals $^{4,16}$. During wheat embryogenesis, bivalency reached its peak at transition 261 stage (DPA 8) and gradually decreased during tissue differentiation (Fig. 3f). This is 262 mostly due to the turbulence of H3K27me3, since H3K4me3 generally didn't change 263 much (Fig. 3f). In both proximal and distal bivalent regions, we observed widespread 264 ATAC-seq, H3K27ac, H3K4me1, H3K9me3, Pol II and H2A.Z signals, but not 265 transcript products and transcription-associated H3K36me3, suggesting a poised state 266 (Fig. 3g). Of note, genes encoding transcription factors are more enriched than others 267 in bivalent regions at every embryonic stage, indicating that H3K27me3 and 268 bivalency are indispensable for accurate transcriptional regulatory circuitry (Fig. 3h). 269 Besides, subgenomes of hexaploid wheat showed a different abundance of histone 270 modifications even at collinear regions. Interestingly, $\mathrm{H} 3 \mathrm{~K} 27 \mathrm{me} 3$ and $\mathrm{H} 3 \mathrm{~K} 9 \mathrm{me} 3$ 271 exhibited higher variation among different sub-genomes than active histone marks 272 such as H3K27ac and H3K4me3, as well as histone variant H2A.Z (Fig. 3i and 273 Supplementary Fig. 5b).

274 Taken together, the histone modification reprogramming is distinct in plants as 275 compared to their counterparts in animals (Fig. 3j), indicating a divergent function of 276 individual histone marks in driving the embryogenesis process in two kingdoms. As 277 well, different subgenomes have varied histone modification, especially H3K27me3. 


\section{Chromatin regulation of maternal gene silence and zygotic gene} activation upon fertilization

Upon fertilization, differentiated parental gametes fused and converted into a totipotent zygote, including maternal gene silencing and zygotic genes initiation with

282 epigenetic reconfiguration in both animals and higher plants ${ }^{8,15,43}$. A large amount of 283 differential expressed genes (DEG) was captured upon fertilization (DPA2/ DPA0), 284 with 8,079 down-regulated genes, mainly involved in maternal tissue identity 285 maintaining such as AP2 and NF-YC9, as well as 8,380 up-regulated genes, including 286 cell cycle and cytokine (CK) signaling genes associated with zygotic initiation (Fig. 287 4a). We next investigate the chromatin landscapes for seeking of driving force for 288 those DEGs. Consistently, active histone modification H3K27ac and H3K4me3 289 suffered an overall lopsided decrease at down-regulated genes, while nearly no 290 change at up-regulated genes (Fig. 4b). By contrast, chromatin accessibility didn't 291 change much for down-regulated genes but gained at up-regulated genes (Fig. 4b). As 292 for H3K27me3, regardless of up or down-regulated genes, a reduction upon 293 fertilization was observed. Such profiles indicated that loss of active histone 294 modification might contribute to genes down-regulation, while gain of chromatin accessibility may trigger gene activation. Indeed, attenuation of either or both

$296 \mathrm{H} 3 \mathrm{~K} 27 \mathrm{ac}$ and H3K4me3 is extensively enriched for down-regulated genes (Fig. 4c, d),

297 with more remarkable down-regulation for loss of both. H3K27ac reprogramming is 298 more determinable compared with H3K4me3 (Fig. 4c, d). Genes subjected to both 299 active histone marks decreasing and transcription levels attenuation are mainly 300 maternal silenced genes, such as orthologs of floral homeotic gene AP2, 301 signaling-related gene MAPKKK17, and stigma expressed gene IAA1 (Fig. 4e, h). 302 Whereas, de novo building of pACRs showed a significant overlap and correlation 303 with genes up regulated (Fig. 4f, g). These genes were enriched for pathways in DNA 304 replication, cell cycle, heterochromatin, etc., which are the cornerstones of the early 
zygotic building (Fig. 4e, h).

Besides, there are still 6,737 genes, nearly $80 \%$ of up-regulated genes upon

307 fertilization, which can't be explained by either chromatin accessibility or histone 308 modification change (Fig. 4f). We thus speculate a hierarchy transcription regulation 309 network (TRN) might govern the sequential activation of ZGA genes, like the case in 310 animals ${ }^{44,45}$. We constructed such TRN based on top-down and bottom-up methods 311 via integrating the ATACseq and RNAseq data (Method; Fig. 4i and Supplementary 312 Fig. 6b). Three TFs, one TCP and two MYBs were on the top layer of the pyramid 313 (Fig. 4i). The footprint of TCP and MYBs showed not only an augmenting binding 314 strength after fertilization but also transcriptional activators' character, generating 315 robust flanking accessibility without change of the footprint depth $(\text { Fig. } 4 j)^{38}$. In 316 addition, the basal targets, which were activated at DPA2 or DPA4, were extremely 317 conserved with more than $80 \%$ of which have orthologs in Arabidopsis ZGA genes 318 set $^{8}$, including genes involved in heterochromatin, cell wall, and cytokinesis (Fig. 4i). 319 We further verified the specific expression pattern of TCP during MZT process by in 320 situ hybridization (Fig. 4k), and its activation of downstream targets $G R A S / N F-Y B$ by 321 reporter assay (Fig. 41).

Taken together, the silence of the maternal genome is attributed to the 323 diminishing of active modification on genes, while the trigger of zygotic may own to 324 de novo building of chromatin accessibility and hierarchical regulation of TFs, with 325 several potential key TFs such as TCP being engaged.

\section{Reprogramming of $\mathbf{H} 3 \mathrm{~K} 27 \mathrm{me} 3$ coordinates with chromatin} accessibility to ensure pre-embryo development

Following fertilization, several cell fate determination genes were gradually activated in pre-embryo, such as genes in WOX and ARR families (Fig. 1c, d). In 330 parallel, we observed a dramatic drop of H3K27me3 at DPA4, but progressively 
331 re-build until DPA8 (Fig. 3e, f, j, Fig. 5a and Supplementary Fig. 7a). For

332 developmental essential genes such as LEC1, BBM, WOX11 and ARR12, local

333 chromatin environments were permissive in pre-embryo (DPA4), with decreasing of

334 repressive mark $\mathrm{H} 3 \mathrm{~K} 27 \mathrm{me} 3$ and increasing of active marks H3K27ac and ATAC

335 signal, while a limited change of H3K4me3 (Fig. 5b). What is more, about $90 \%$ of the

336 H3K27me3 erasure regions at pre-embryo were re-built in transition stage, along with

337 a large amount of de novo gain of H3K27me3 regions (Fig. 5c). The erasure of

338 H3K27me3 was associated with CK pathway genes, embryo essential genes, and

339 tissue specification genes, while the few augment was involved in auxin-related gene

340 sets (Fig. 5c and Supplementary Fig. 7b, c). Correlated with the massive gain of

341 H3K27me3 at DPA8, TaCLFs, a methyltransferase for H3K27, were temporally

342 activated around DPA8 (Supplementary Fig. 7d), and several functional tested 'PRE'

343 type motifs ${ }^{46,47}$ were enriched at $\mathrm{H} 3 \mathrm{~K} 27 \mathrm{me} 3$ restoration loci with their cognate

344 binding TFs highly expressed at DPA8 (Supplementary Fig. 7e, f). Thus, cis-motif and

345 trans-factors mediated H3K27me3 deposition may be involved in embryonic

346 development in wheat.

H3K27me3 intensity generally showed a negative correlation with transcription

348 (Fig. 5d and Supplementary Fig. 2b), however, decreasing of H3K27me3 alone could 349 not guarantee gene activation from DPA2 to DPA4 (Fig. 5e). However, H3K27me3

350 reduction was highly overlapped with the addition of $\mathrm{H} 3 \mathrm{~K} 27 \mathrm{ac}$ or chromatin 351 accessibility (Fig. 5e). As expected, genes with a gain of active chromatin 352 modification in addition to $\mathrm{H} 3 \mathrm{~K} 27 \mathrm{me} 3$ reduction showed significant activation, with a 353 more dramatic effect by a gain of chromatin accessibility (Fig. 5e). Furthermore, there 354 were 561 genes whose transcriptional activation showed highly synchronous patterns 355 with the gain-of-chromatin after DPA4 (Fig. 5f and Supplementary Fig. 7g). This 356 synchronization ensured the proper timing of genes activation, with embryo essential 357 gene LEC1 firstly activated followed by pluripotency gene ARR12, and later 358 differentiation genes $X M X L 5$ and IPS2 (Fig. 5f and Supplementary Fig. 7g). Together, 
this suggested that resetting of $\mathrm{H} 3 \mathrm{~K} 27 \mathrm{me} 3$ lifted the chromatin layer barrier on

360 embryo patterning genes, which was progressively activated along with the gain of

361 chromatin accessibility. Alternatively, a global regain of H3K27me3 was observed at

362 DPA8, a transition stage with the maximal open chromatin and bivalent regions as

363 well (Fig. 1f, 3f, 5a, c, g and Supplementary Fig. 7a). Indeed, among the 133,716

364 gained H3K27me3 peaks, 40\% show bivalency status with enriched H3K4me3 and 365 opened chromatin status (Fig. 5g, h). The expression levels of genes with H3K27me3

366 marked promoters were significantly lower compared with bivalency promoters at 367 transition stage, while slight change was detected between genes with distal 368 H3K27me3 and bivalency (Fig. 5g, i). Genes got bivalency or H3K27me3 at both 369 promoter and distal regions were silenced at transition stage, with stronger 370 suppression function of modifications at promoter compared with those at distal (Fig. $3715 \mathrm{~g}, \mathrm{i}, \mathrm{j})$. Yet, genes with bivalent promoters still have the opportunity to re-activation, 372 such as $B B M$, but not genes with H3K27me3, such as ARR12 (Fig. 5i, j).

Thus, the global depletion of H3K27me3 in pre-embryo resulted in a permissive

374 chromatin environment, where the timely opening of chromatin can trigger the 375 activation of developmental genes. Bivalency modification generated by H3K27me3 376 restoring marked cell fate determination genes, whose dysregulation was related to 377 transition of totipotent pre-embryo to differentiation.

\section{Gene regulatory network orchestrates embryo pattern formation}

After transition stage, embryo pattern formation was initiated, along with 380 precisely programmed transcription regulation pyramid ${ }^{48}$. The complex relationship in 381 TF-genes and TF per se could be well exemplified by the continuous transcription 382 trajectories (Fig. 6a, b). To test how the architecture of embryo patterning from 383 totipotent stem cell to maturation, we calculated the pseudotime of stages covering 384 early- to mature-embryo based on the PCA distance (Fig. 6b). As expected, an 385 excellent association between gene expression and both pACR and dACR was 
observed (Fig. 6c), which indicated that the expression pattern of these genes was governed by individual ACRs and TFs binding. Comparison of genes expressed in the early time point (Cluster 1 and 2) and the later ones (Cluster 3 and 4) presented a

389 functional diversity, of which the early elevated genes were involved in cell division,

390 while the later ones in cell specification (Fig. 6d). What is more, motifs of TFs

391 binding sites, whose orthologs can facilitate inducement of cell totipotency in 392 Arabidopsis, were enriched in early build ACRs, such as the binding motifs of LEC1,

393 MYB118, and WUS, etc. Whereas the TFs, which function in seed dormancy, mainly 394 occupied the later elevated ACRs, such as ABI5 (Fig. 6e). This result demonstrated evolutionary conservation of several key factors in embryo patterning in monocot and 396 eudicot.

We further dissect the architecture of the gene regulation network (GRN). In 398 addition to binding motif specificity, we filtered target genes to TFs by co-expression 399 pattern as well (Method; Fig. 6f). As a result, 1,158 paired TF-cis motif interactions 400 were identified including 154 target genes, 695 ACRs and 191 TFs (Fig. 6f). To better 401 understand how TFs are coordinated during embryo pattern formation, we next extract 402 the TF-TF regulation network (Fig. 6g). We found four pivotal classes of TFs located 403 at the center of the network, TCPs, ARFs, MYBs, and WOXs. Some of those TFs 404 such as WOXs and ARFs are reported to regulate embryo patterning in other 405 species ${ }^{49-51}$. Several known genes regulating organ and tissue specification in 406 Arabidopsis were under the governing of these TF modules, such as IAA14, LAX2, 407 PLT1, and SMXL5, etc. To further estimate this GRN, we use in situ hybridization to 408 verify the spatiotemporal expression pattern of key TFs module including 409 LEC1-Myb118-ZHD5-LEC2-BBM. As shown, the LEC1, Myb118 and ZHD15 genes 410 were highly expressed in pre-embryo stages of overlapped tissue, while LEC2 was 411 activated later, with a peak at transition stage, and $B B M$ gradually induced at 412 mid-embryo, indicating a sequential pattern (Fig. 6h). The luciferase activity from the 413 reporter assay validated the regulatory circuit along LEC1, MYB118, ZHD5, LEC2 
414 and BBM module (Fig. 6i). Interestingly, TaLEC2 could bind the pACR of $B B M$

415 directly and activate it in vitro (Fig. 6i), which is not reported in the model plant 416 Arabidopsis.

417 Thus, these data described a cohesive, sequential wave of TFs regulation in 418 governing wheat embryo pattern formation.

419 Chromatin regulation facilitates embryo maturation and prohibits 420 extensive organogenesis

During seed maturation, nutrient was accumulated in both endosperm and embryo, meanwhile, the dormancy program was progressively established with increased ABA levels accompanied by desiccation ${ }^{52}$. Indeed, genes elevated

424 specifically in late embryo development were enriched in carbohydrate, lipid, protein, 425 amino acid, secondary metabolic pathway, and ABA signaling and homeostasis (Fig. 426 7a). As expected, H3K27ac increased progressively at those genes during late embryo 427 development, but H3K27me3 declined to a relatively low level, while chromatin 428 accessibility was slightly changed (Fig. 7b). Next, we applied ATAC footprint to seek 429 candidate TFs associated with the regulatory regions of the above genes. ABA-related 430 TFs were enriched for regulating ABA pathway genes, which verified the logic of this 431 analysis (Fig. 7c). In addition, most nutrient storage pathways shared at least one 432 upstream TF with ABA pathway, such as ABI5 and ABF1 (Fig. 7d), which are known 433 to regulate a subset of late embryogenesis abundant genes in Arabidopsis $^{53}$. This 434 result suggested that several key regulators could synchronize nutrient accumulation 435 and embryo dormancy in cooperation with chromatin landscape dynamics.

436 Unlike animals, extensive organogenesis is prohibited during late embryo 437 development, which enables plants to generate post-embryonic lateral organs in 438 adaptation to changeable environments. Meanwhile, immature embryo served as 439 excellent explants for wheat regeneration, a process including regaining of totipotency 
440 and organogenesis ${ }^{53,54}$, however such capability was reduced upon embryo maturation

441 (Supplementary Fig. 8a). We wonder how the totipotency capability is attenuated and

442 why the terminal organ is not formed during embryo maturation. First, we identified

443 organ identity genes by their specific expression in terminal organs such as root and

444 leaf, for comparison totipotent genes were characterized with high expression in 445 mid-embryo or embryonic callus (Fig. 7e). GO enrichment of different groups of 446 genes verified the accuracy of genes identity (Supplementary Fig. 8b). Next, we 447 evaluated the chromatin landscape of each cluster genes in different organs. Depletion 448 of $\mathrm{H} 3 \mathrm{~K} 27 \mathrm{me} 3$ on organ identity genes was observed at the respective organ, whereas, 449 an obvious ATAC signal presented for both totipotency and organ identity genes in its 450 corresponding organ (Fig. 7f, Supplementary Fig. 8c, d). Surprisingly, low/no 451 H3K27me3 was enriched on totipotent genes even in differentiated organs (Fig. 7f). 452 In embryonic callus, of which new plant will regenerate, the promoter region of both 453 totipotent genes and organ identity genes were opened with high chromatin 454 accessibility and low H3K27me3 level (Fig. 7f). Thus, chromatin accessibility and 455 H3K 27 me3 coordinately regulated organ identity gene's activation potential while 456 chromatin accessibility but not $\mathrm{H} 3 \mathrm{~K} 27 \mathrm{me} 3$ mediated repression is indispensable for 457 totipotency gene silencing. Indeed, several regeneration-related factors, such as REV, 458 SAUR41, CKX7 and DDM1 were gradually diminished during embryo maturation 459 along with closing chromatin status but activated during callus induction process (Fig. $4607 \mathrm{~g}$ ). Last, we investigated the gene-regulatory logic underlying plant organ formation. 461 WRKYs and AtHB TFs footprint were enriched in regulatory regions of root and leaf 462 identity genes respectively, whereas, bHLH and GATA TFs footprint were enriched in 463 both gene-sets (Supplementary Fig. 8e). Remarkably, nearly half of the identified TFs 464 did not show organ-specific expression, but instead a wide-spectrum pattern, 465 indicating that TFs expression per se cannot determine the specificity of organ 466 identity (Supplementary Fig. 8f). For example, although WRKY75 was expressed 467 during embryo maturation and it owns the capability to bind and active $A M T 1 ; 1$, an 
ammonium transporter coding gene in root (Fig. 7h, Supplementary Fig. 8f), AMT1;1 was silenced due to the H3K27me3 repression and compaction chromatin environment at the promoter (Fig. 7i).

Taken together, chromatin layer regulation ensures the proper embryo maturation process, with an accumulation of nutrients and reduced totipotency ability but not extensive organogenesis.

\section{Epigenetic regulated ontogenetic divergence and inverse hourglass} pattern during embryogenesis

As wheat is an allohexaploid plant, we further extend the understanding of epigenetic regulated embryogenesis to the evolution and polyploidization perspectives. Genes were clustered based on their evolutionary age (Supplementary Fig. 10a). Most of the genus Triticum-specific genes, especially D-subgenome unique genes, were located within PcG chromatin state and did not participate in embryonic development (Supplementary Fig. 10a-c). For the homeolog triads that are present in all three subgenomes, chromatin accessibility is related to the bias expression pattern (Supplementary Fig. 11) in addition to histone modifications as reported ${ }^{55}$. In detail, suppressed triads are generally more than dominant triads, in addition, D-subgenome suppressed triads are relatively less than A or B-subgenome suppressed. More balanced triads were expressed in the middle period compared with early and late embryogenesis (Supplementary Fig. 10d). Such observation was generally consistent with the previous report but different in details ${ }^{27}$.

Polyploidy may drive genes neo- or sub-function to confer phenotypic plasticity, which can be represented by the varied expression pattern. Instead of identifying genes of differential expression at a specific embryonic stage by comparing hexaploid wheat with tetraploid and diploid ${ }^{27}$, we look for genes showing varied expression profiles during the process of embryo development. Indexing by Pearson correlation 
494 degree, genes are categorized into dysfunction, middle, and conserved clusters (Fig 8a 495 and Supplementary 9a). As expected, conserved genes were mostly homeologs triads, 496 with sub-genome balanced expression, and showed higher sequence conservation and 497 stronger selection pressure (Fig. 8b, c and Supplementary Fig. 9b-f). However, a 498 considerable proportion of dysfunctional genes changed expression profile in 499 hexaploid wheat as compared to ancestors, but with slight sequence variation and 500 subject to strong negative selection (Fig. 8c). Promoters of these genes, especially 501 around $3 \mathrm{~Kb}$ upstream of TSS, were preferably inserted by TEs (Fig. 8d and 502 Supplementary Fig. 9g). Intriguingly, TE insertion regions tended to be accessible 503 chromatin and enriched for H3K27ac and H2A.Z (Fig. 8e). For example, 504 TaUGT91C1 showed a B-subgenome dominance expression in hexaploid wheat, 505 while silenced in all ancestors (Fig. 8f). Accordingly, the promoter and dACR region 506 harbored TEs with higher chromatin accessibility as compared to A or D counterparts. 507 Therefore, the TEs insertion within proximal and distal active chromatin regions may 508 drive the novel expression pattern of offspring genes following polyploidization.

A previous study on embryogenesis revealed a conserved hourglass model in 510 hexaploid wheat as well as its tetraploid, and diploid ancestry, but a slight difference 511 for the phylotypic stage ${ }^{27}$. Here, our transcriptome data in hexaploid wheat (Chinese 512 Spring) also supported that transition stage of DPA8 represents the phylotypic stage 513 (Supplementary Fig. 9h). In addition, we find an inverse hourglass model for 514 transcriptional variability. A sharp mid-developmental transition was presented, which 515 separated two conserved phases, early and late developmental stages in both the 516 comparison between homoeologs in hexaploid per se and between counterparts of 517 hexaploid gene and its ancestors (Fig. 8a, g and Supplementary Fig. 9a). We 518 hypothesized that epigenetic regulation may account for this middle-stage transition. 519 Indeed, H3K27ac was mainly contributed to the middle stage rather than early- and 520 late-stage, whereas H3K27me3, H3K4me3, and ATAC behaved oppositely (Fig. 8h 521 and F Supplementary Fig. 9i). In addition, chromVAR analysis indicated that the key 
522 TFs during embryogenesis functioned mainly in the middle stage (Fig. 8h and F

523 Supplementary Fig. 9j). Thus, the cis- and trans- regulation driven by TFs was more 524 meaningful in mid-stage compared with early- and late-stage. In sum, transcriptional 525 variance showed an inverse hourglass model, which may be governed by chromatin 526 layer regulation at early and late stage, while cis-trans regulation at the middle stage 527 (Fig. 8i).

\section{DISCUSSION}

Despite containing the same genome, different cell types possess unique

530 identities with specific gene expression patterns. This is partly because some unique

531 portions of the genome are accessible in each cell type, which is controlled at the level 532 of chromatin. During embryogenesis, parental differentiated gametes fuse and convert 533 into a totipotent zygote, and then to a differentiated embryo, cells undergo extensive 534 identity switch, and thus provide an ideal system for studying chromatin-based 535 transcription regulation. Indeed, embryogenesis is extensively studied in mammalians. 536 However, our understanding of embryogenesis is far lagged in plants, especially in 537 crops. In this study, we generated comprehensive RNAseq, CUT\&Tag and ATACseq 538 data in three early embryo sac and five later embryo samples of hexaploid wheat 539 Chinese Spring. It provides a genome-wide annotation of dynamic 'reference 540 epigenome', which enables the comparative analysis of large-scale chromatin 541 reprogramming and its function for embryogenesis between plants and animals, and 542 the understanding of multi-layer orchestrated transcriptional regulation circuit at 543 different stages of embryonic development in wheat. Integration with the previous 544 report on the transcriptome of embryogenesis from different ploidy wheat ${ }^{27}$, our study 545 further revealed the potential driving force for evolutionary divergence among 546 different sub-genome in shaping embryogenesis in polyploidy wheat.

In this study, we observed large-scale chromatin modification reprogramming 
549 (Fig. 3e, j, Fig. 4b, Fig. 5a and Supplementary Fig. 5a). Though the histone 550 modification resetting pattern shows general similarity to animals $\mathrm{s}^{4,16}$, there was a large 551 variation in terms of modification types and timing of reprogramming. In wheat, 552 widespread H3K27ac dropped off during MZT and contributed to the silence of 553 maternal genes, slightly for $\mathrm{H} 3 \mathrm{~K} 4 \mathrm{me} 3$ (Fig. 3e, $\mathrm{j}$ and Fig. 4b), which however is 554 dramatically reset upon fertilization and re-build during major ZGA and is importan 555 for ZGA in mice ${ }^{4,15}$. H3K27me3 sharply dropped at pre-embryo but not right after 556 fertilization like its counterpart in mammals ${ }^{4}$ (Fig. 3e, j, Fig. 5a and Supplementary 557 Fig. 5a). Both H3K27ac and H3K27me3 were momentarily and universally 558 re-established, of note, regions of H3K27me3 decreased in DPA4 is a great part of that 559 increased in DPA8 (Fig. 3j, Fig. 5a and Supplementary Fig. 5a). This suggests the 560 epigenome 'rebooting' model is conserved for both animals and plants ${ }^{15}$. Importantly, 561 H3K27me3 dynamics marked embryonic essential genes (Fig. 3h and Fig. 5b), 562 indicating its pivotal role in embryo development. Indeed, we found aberrant seed 563 development when knocking out H3K27me3 writer complex component 564 (Supplementary Fig. 12), though a detailed mechanism still needs to address. Recent 565 findings characterize the direct involvement of PRC2 component, writer of 566 H3K27me3, for embryo development in Arabidopsis ${ }^{56}$. Besides histone modification, 567 chromatin accessibility also shows dynamics during embryogenesis. Interestingly, 568 dACRs (account for 75\% total ACRs) and pACRs (16\%) show unique fluctuation 569 pattern, correlated with distinct transcriptional regulation respectively (Fig. 2b). For 570 genic regions, pACRs were significantly gained upon fertilization, strongly correlated 571 with zygotic gene activation (Fig. 4b, e-h), and continued gain till pre-embryo (DPA4) 572 then gradually declined later on. Whereas, dACRs gradually gained after fertilization, 573 with 'transient' maximize at transition stage (DPA8) then quickly declined later, 574 where the opened intergenic regions are highly overlapped with multiple TEs, such as 575 Gypsy and CACTA, as well as 'burst' of H3K9me3 region at centromere and telomere. 576 Further analysis of global DNA methylome and enhancer RNA profiling around 
577 transition stage would give a clearer conclusion of the functional consequence of such

578 dACR eruptions. Nevertheless, our data provide a novel comparison between animals

579 and plants for the whole embryogenesis process driven by chromatin landscape 580 dynamics.

581 Embryogenesis is governed by precise transcriptional regulation program ${ }^{2,3,57}$.

582 However, how such transcription regulation is orchestrated by different types of 583 regulation, such as histone modification, chromatin accessibility, DNA methylation, 584 transcription factor-cis motif modules, is not clear. Based on our analysis, a dynamic 585 'three-layer regulation model' is proposed for different embryonic development stages 586 (Fig. 8i). In this model, de-repression of local histone modification is a prerequisite of 587 permissive chromatin environment and open chromatin is the cornerstone of 588 transcription factors free binding. At pre-embryo, H3K27me3 was globally reset, 589 which provides a flexible chromatin environment, but yet, not sufficient to active genes 590 and gain-of accessibility at the promoters are the indispensable triggers. After this, 591 freestanding TFs coordinate was gradually built in a subtle and specific manner (Fig. 5).

592 The attenuation of totipotency and prohibition of extensive organogenesis at 593 late-embryo development stage can also support this model in an opposite way (Fig. 594 7e-i). A representative case is the WKRY75-AMT1;1. Despite the transcriptional 595 activation of WKRY75 for $A M T 1 ; 1$ in reporter assay, AMT1;1 was still repressed in 596 late-embryo because of the compacted promoter region along with enriched 597 H3K27me3. Besides the stage-specific cases, similar multi-layer regulation could 598 apply to the entire embryogenesis process in general. Transcription data for 599 embryonic development demonstrate a cross-kingdom and ploidy conserved 'inverse 600 hourglass model', in which a transcriptional divergent mid-developmental transition 601 punctuated the conserved early- and late-stage (Fig. 8a, g and Supplementary Fig. 9a). 602 Accordingly, a panorama of genetic and epigenetic dynamic presents that global 603 histone modification reprogramming at early-stage, while the chromatin accessibility 604 burst and TF-binding at mid-stage as well as the relative compacted genome at 
605 late-stage (Fig. 4-7, Fig. 8h-i and Supplementary Fig. 9i, j). Thus, the early- and 606 late-stage were dominated by chromatin level restriction, while mid-stage was 607 governed by TF-motif regulatory circuits along with open chromatin, of which the 608 chromatin level regulation effect is minimized. This regulation pattern fits the 609 transcriptional divergent 'inverse hourglass' in general, in combination with the 610 genetic diversity 'hourglass model' for mediating conserved and diversified 611 embryogenesis in different species.

612 Bread wheat (AABBDD) undergoes polyploidization and domestication, along 613 with evolutionary divergence among different sub-genomes. Indeed, we observed 614 dynamic biased expression of homeolog triads during embryogenesis, correlated with 615 histone modification variations as well as chromatin accessibility differences among 616 A,B, D sub-genome (Fig. 2d, Fig. 3i, Supplementary Fig 3d and Supplementary Fig 617 5b). In addition, a considerable proportion of genes showed varied expression profiles 618 during embryogenesis in allohexaploid bread wheat compared with the diploid 619 ancestors $^{27}$ (Fig. 8a and Supplementary Fig 9a). Part of them possesses DNA 620 variations within the genic regions, which may alter gene function and indirectly 621 change their expression profile as an adaptation for the 'new' roles. Whereas other genes with slight or nearly no sequence variation at genic region still show altered expression patterns. Of note, promoters and distal regulatory regions of those genes contain asymmetric TE insertions among different sub-genomes, with a preference for sites of open chromatin region and enriched for active histone marks such as H3K27ac and H2A.Z. Thus, epigenetic landscape variation among different 627 sub-genome, especially TE inserted active chromatin regions mediated transcriptional 628 alteration, may drive the evolutionary divergence in shaping embryogenesis in 629 polyploidy wheat.

630 Besides, our data also elucidated detailed transcriptional regulation circuits 631 during ZGA and embryo pattern formation, as well as the maturation of embryo (Fig. 632 4, Fig. 6, Fig. 7). The hierarchy transcription regulation pyramids and underlying 
633 epigenetic regulation of key transcription factors during ZGA would be worth to test

634 for the automatic embryo development program without fertilization. As well, the

635 knowledge about programmed embryo pattern formation and gradually attenuation of 636 totipotency would be valuable for studying wheat regeneration, during which 637 immature embryo serves as the excellent explants. As a comprehensive study of 638 embryonic chromatin landscape dynamics and transcriptome, our research is 639 anticipated to facilitate gene functional study of wheat embryogenesis and plant 640 breeding application, such as parthenogenesis and somatic embryogenesis.

\section{Methods}

\section{Plant materials and growth conditions}

643 A spring wheat (Triticum aestivum) Chines spring was used in this study. All plant was 644 grown in a growth room under a $16 \mathrm{~h}$ light $/ 8 \mathrm{~h}$ dark cycle with light intensity $1,000 \mu \mathrm{mol}$ $645 \mathrm{~m}-2 \mathrm{~s}-1$, and temperature $15-26^{\circ} \mathrm{C}$ depending on growth stage. The stamens are 646 removed before the pollen matures. Then we conduct artificial pollination and record 647 the number of days to ensure the accurate time of seed development. Embryo and 648 embryo sac at the specific developmental stage were sampled using RNA and nuclear 649 extraction.

\section{RNA extraction}

651 Total RNA was extracted using HiPure Plant RNA Mini Kit according to the 652 manufacturer's instructions (Magen, R4111-02), and libraries were sequenced using 653 an Illumina Novaseq platform.

\section{CUT\&Tag experiment}

655 The CUT\&Tag experiment was performed as reported with minor modifications ${ }^{35}$. For 656 each replicate, the fresh samples were soaked in the HBM buffer $(25 \mathrm{mM}$ Tris- $\mathrm{Cl} \mathrm{pH}$ 
657 7.6, $0.44 \mathrm{M}$ sucrose, $10 \mathrm{mM} \mathrm{MgCl2,} \mathrm{0.1 \%} \mathrm{Triton-X,} 10 \mathrm{mM}$ Beta-mercaptoethanol, 2 $658 \mathrm{mM}$ spermine, $1 \mathrm{mM}$ PMSF, EDTA-free protease inhibitor cocktail), after chopped 659 with a razor blade, the mixture was filtered through a $40-\mu \mathrm{m}$ cell strainer. The crude 660 nuclei was washed twice by HBB buffer $(25 \mathrm{mM}$ Tris-Cl $\mathrm{pH} 7.6,0.44 \mathrm{M}$ sucrose, 10 $661 \mathrm{mM} \mathrm{MgCl2}, 0.1 \%$ Triton-X, $10 \mathrm{mM}$ Beta-mercaptoethanol). Then the crude nuclei 662 were stained with 4,6-diamidino-2-phenylindole and counted by hemocytometer. HEPES pH 7.5; 150 mM NaCl; 0.5 mM Spermidine; 1× Protease inhibitor cocktail; 2 mM EDTA; 0.1\% BSA) containing a 1:50 dilution of corresponding antibody. After overnight incubation in $4^{\circ} \mathrm{C}$, the primary antibody was removed by centrifuged $(1,200 \mathrm{~g}, 3 \mathrm{~min})$, the nuclei were incubated in $50 \mu 1$ wash buffer (20 mM HEPES pH 7.5; $150 \mathrm{mM} \mathrm{NaCl} ; 0.5 \mathrm{mM}$ Spermidine; $1 \times$ Protease inhibitor cocktail) with secondary

669 antibody (1:100; Guinea Pig anti-Rabbit IgG antibody) at $4^{\circ} \mathrm{C}$ for around $1-2$ hour and 670 then wash twice with wash buffer. A 1:100 dilution of pA-Tn5 complex was prepared in 671 CT-300 buffer (20 mM HEPES pH 7.5; $300 \mathrm{mM} \mathrm{NaCl} ; 0.5 \mathrm{mM}$ Spermidine; 1× 672 Protease inhibitor cocktail), after nuclei centrifuged, add $100 \mu 1 \mathrm{mix}$ and incubate $2-3 \mathrm{~h}$ 673 in $4^{\circ} \mathrm{C}$. After incubation with pA-Tn5, wash twice with CT-300 buffer. Then incubate 674 nuclei in $300 \mu$ l Tagmentation buffer (20 mM HEPES pH 7.5; $300 \mathrm{mM} \mathrm{NaCl} ; 0.5 \mathrm{mM}$ 675 Spermidine; $1 \times$ Protease inhibitor cocktail; $10 \mathrm{mM} \mathrm{MgCl2}$ ) in $37^{\circ} \mathrm{C}$ for $1 \mathrm{~h}$. To stop 676 tagmentation reaction, add $10 \mu \mathrm{l} 0.5 \mathrm{M}$ EDTA, $3 \mu 110 \%$ SDS and $2.5 \mu 120 \mathrm{mg} / \mathrm{ml}$ 677 Protease $\mathrm{K}$, incubate $1 \mathrm{~h}$ in $50{ }^{\circ} \mathrm{C}$. The DNA was extracted with 678 phenol:chloroform:isoamyl alcohol, precipitated with ethanol and resuspended in 679 ddH2O. The library was amplified 17 cycles by Q5 high fidelity polymerase (NEB, 680 M0491L), and purified by AMPure XP beads (Beckman, A63881). Finally, the library 681 was sequenced using an Illumina Novaseq platform. 
683 The method of nuclei extraction was performed as before. After checking the nuclear 684 integrity, the nuclei extracted (50,000 per reaction) were incubated with the Tn5 685 transposase and tagmentation buffer at $37^{\circ} \mathrm{C}$ for $30 \mathrm{~min}$ (Vazyme Biotech,TD501-01) ${ }^{34}$. 686 After tagmentation, the DNA is purified by PCR purification kit (QIAGEN, 28106). 687 PCR was performed to amplify the library for 9-12 cycles using the following PCR 688 conditions: $72{ }^{\circ} \mathrm{C}$ for $5 \mathrm{~min} ; 98{ }^{\circ} \mathrm{C}$ for $30 \mathrm{~s}$; and thermocycling at $98{ }^{\circ} \mathrm{C}$ for $15 \mathrm{~s}, 63{ }^{\circ} \mathrm{C}$ 689 for $30 \mathrm{~s}$ and $72{ }^{\circ} \mathrm{C}$ for $40 \mathrm{~s}$; following by $72{ }^{\circ} \mathrm{C} 5 \mathrm{~s}$. After the PCR reaction, libraries were purified with AMPure beads (Beckman, A63881).

\section{Bioinformatics Data Preprocessing and Alignment}

692 All fastq data, including DNA and RNA sequencing, were generated based on Illumina 693 Hiseq-PE150. Raw data were filtered by fastp (v0.20.0) with “--detect_adapter_for_pe" 694 parameter for reads filter, low-quality bases trimming, and adapters removing ${ }^{58}$. 695 Furthermore, the clean data was evaluated by fastqc software (v0.11.8) (https://github.com/s-andrews/FastQC) to ensure the high quality of reads.

Both DNA sequencing including resequencing, CUT\&Tag, and ATAC-seq and 698 RNA sequencing data were aligned based on Triticum aestivum (Chinese Spring) 699 genome assembly (IWGSC RefSeq v1.0) ${ }^{31}$, which was downloaded 700 from https://urgi.versailles.inra.fr/download/iwgsc/IWGSC_RefSeq Assemblies/v1.0/.

701 The IWGSC Annotation v1.1 was used as the gene annotation reference. For DNA 702 sequencing data, BWA-MEM (v0.7.17) algorithm was used for alignment with "-M" 703 parameter to avoid shorter split hits ${ }^{59}$. For RNA sequencing data, hisat2 (2.1.0) was 704 applied for reads mapping with default parameters ${ }^{60}$.

\section{RNA-seq Data Processing and Expression Clustering}

706 Sam files generated from hisat2 were converted to bam files without deduplication. 
707 FeatureCount v1.6.4 was used for reads quantity per gene ${ }^{61}$. An R package edgeR was

708 used for DEGs (differentially expressed genes) examination, with a threshold absolute

709 value of Log2 Fold Change $\geq 1$ and FDR $\leq 0.05^{62}$. The raw matrix was further

710 normalized to TPM (Transcripts Per Kilobase Million) for gene expression

711 quantification. Genes expression data from different tissue was downloaded from a

712 previous publication ${ }^{55}$.

713 TPM values of genes were clustered by k-means method in the heatmaps in Fig.

$7145 \mathrm{e}$ and $5 \mathrm{~h}$. Modules information generated by $\mathrm{WGCNA}^{63}$ was used for key gene set 715 selection.

For functional enrichment, GO annotation files were generated from IWGSC

717 Annotation v1.1 and an R package clusterProfiler was used for enrichment analysis ${ }^{64}$.

718 Cut\&Tag and ATAC-seq Data Processing

719 Cut\&Tag data analysis was largely based on the previously provided pipeline ${ }^{65}$. In

720 brief, two replicates sam files were converted to bam files and sorted by samtools, 721 respectively ${ }^{59}$. We further filter the reads mapped with "samtools view -bS -F 1,804-f

7222 -q 30" to filter the low-quality mapped reads. The high-quality mapped reads were 723 reduplicated using Picard-2.20.5-0 ("Picard Toolkit." 2019). Two replicate bam files 724 were merged by samtools. For IGV browser visualization, merged bam files were 725 converted to RPKM (Reads Per Kilobase per Million mapped reads) normalized 726 bigwig files with 10 bp bin size for browser visualization by bamCoverage provided 727 by deepTools (3.3.0) with parameters “-bs 10 --effectiveGenomeSize 14,600,000,000 728 --normalizeUsing RPKM --smoothLength $50^{״ 66}$. For Cut\&Tag data comparison, scale 729 factors were calculated by ChIPseqSpikeInFree (v1.2.4 $)^{67}$. For peak calling, both 730 SEACR v1.3 and MACS2 v 2.1.2 were used ${ }^{68,69}$. We performed SEACR with numeric 731 threshold 0.05 and normalized stringent model. For narrow histone marker (H3K27ac 732 and H3K4me3) and broad histone markers (H3K27me3), parameters "-p 1e-3" and 733 “--broad --broad-cutoff 0.05 " provided by MACS2 were used, respectively. Finally, 
734 only peaks generated by MACS2 which overlapped with that generated by SEACR

735 were retained for downstream analysis by "intersect -wa" parameters of bedtools 736 v2.27.170.

737 The bam file process and bigwig conversion steps in ATAC-seq are the same as 738 that in Cut\&Tag. For ATAC-seq peak calling, only MACS2 was used with parameters 739 “--cutoff-analysis --nomodel --shift -100 --extsize 200”. ChIPseqSpikeInFree was not 740 applied in ATAC-seq data process.

741 For both Cut\&Tag and ATAC-seq peaks, if a peak overlapped with the proximal 742 of a gene, including $3 \mathrm{~kb}$ upstream and $2.5 \mathrm{~kb}$ downstream, we assigned the peak to 743 the gene. If multiple genes meet the condition, a position priority strategy (promoter > 744 exon $>$ intron $>$ 5'UTR $>$ 3'UTR $>$ downstream) and nearest gene principle was used 745 for target genes assign. An R package ChIPseeker was used for this peaks annotation 746 process $^{71}$.

\section{DERs detection}

748 Reads count under special peaks of Cut\&Tag and ATAC-seq were calculated by 749 FeatureCount ${ }^{61}$. For Cut\&Tag data, scale factors generated by ChIPseqSpikeInFree 750 were used for differential analysis following the suggested usage of DESeq2 method 751 in ChIPseqSpikeInFree manual ${ }^{67,72}$.

\section{Chromatin state analysis}

753 For chromatin state analysis, chromHMM was used ${ }^{40,41}$. "BinarizeBam" and

754 "LearnModel" commands were used for chromatin-state annotation. We used 9 755 CUT\&Tag data as input and got 15 chromatin-states (Fig. 3a left panel).

\section{ATAC-seq foorprints identity}

757 HINT (Hmm-based IdeNtification of Transcription factor footprints) was used for 758 ATAC-seq footprints identity ${ }^{73}$. JASPAR Plantae database (https://jaspar.genereg.net/) 
759 was used as motifs $\operatorname{set}^{74}$.

\section{Distal ACRs annotation}

761 The distal ACRs annotation strategy was largely based on a previous study with a 762 small modification ${ }^{38}$. In brief, genes within $0.5 \mathrm{M}$ from a distal ATAC-seq peak are 763 considered candidate target genes (Supplementary Fig. 4b). Then if there is a 764 significant positive correlation between gene expression (TPM) and ATAC-seq signal 765 (FPKM) in eight samples, this gene is considered to be the target of the peak 766 (Supplementary Fig. 4b). A small difference from the Corces et al. (2018) is that we 767 use this strategy only for distal ATAC-seq peaks, and proximal peaks annotation was 768 based on the nearest gene principle ${ }^{38}$.

\section{GRNs construction in early embryogenesis}

770 GRNs construction in early embryogenesis was based on top-down and bottom-up 771 methods (Fig. S5a). We believe that genes whose expression was up-regulated but not 772 promoter ATAC signal may be largely regulated by TFs that both expression and 773 pACRs up-regulated (Fig. 4b left panel). Thus, the GRN contained four layers, in 774 which the lowe two layers were genes firstly up-regulated in DPA2 and DPA4 775 compared with DPA0, respectively. Firstly TFs motif was enriched for the 776 up-regulation (DPA2/DPA0) promoter ATAC-seq peaks that were assigned to 777 up-regulated (DPA2/DPA0) genes using homer software (v4.11) ${ }^{75}$. Two types of 778 transcription factors were enriched, including one TCP and two MYB. We used the 779 top-down method to find their downstream target genes through the footprint of the 780 three TFs. Then we used the bottom-up method to find the upstream TFs of genes in 781 the lower two layers through motif enrichment by clusterProfiler ${ }^{64}$. Finally, we got the

782 TFs in the second layer through the intersection of the targets of the top layer and the 783 enrichment result of the bottom layers. These TFs were both expression up-regulated 784 and pACR signal up-regulated. In most conditions, one motif corresponds to multiple 
785 TFs. To get the specific TFs, we overllaped our GRN result with previous Genie3 786 network $^{55}$ and got the final GRN in Fig. 4h. EMB genes were got from a previous 787 review in Arabidopsis $^{76}$, and we used diamond (v2.0.13) with parameter “--id 50 -e 788 1e-20 -f 6" $6^{, 77,78}$.

\section{Psuedotime indexing and GRNs construction in embryo body}

\section{0 formation}

791 Only six-module genes generated from WGCNA were used for the analysis in Fig. 6.

792 The psuedotime indexing method is the same as previous studies ${ }^{79-81}$. Briefly, 793 principal component analysis

PCA was used for both RNA- and ATAC-seq data for specific genes. The psuedotime was calculated based on the sample distance between neighbor samples and was scaled to a range from 0 to 10 . For one gene, the expression model was fit based on expression level and psuedotime using the "loess" function in R, and

798 500-time points were generated between 0 to 10 , as well as the corresponding 799 expression levels based on the fitted curve.

For one gene, the expression level was normalized by Z-score. We further 801 calculated the PC1 and PC2 for every gene using the expression values of eight 802 samples. Because the standard expression values of all genes can form a circle, the 803 atan 2 function in $\mathrm{R}$ was used to return the angle in radians for the tangent PC2/PC1, 804 which were further used for gene expression order ranking. As a result, the psuedotime expression of genes was ranked and visualized by complexHeatmap in

$806 \mathrm{R}^{82}$. GO and motif (generated from footprint analysis) enrichment was calculated by 807 clusterProfiler ${ }^{64}$.

808 For GRN building, the TFs for significantly enriched motifs with $p$-value $<0.05$ 809 were used. We further calculated the Pearson correlation between TFs and 810 corresponding targets, and only significant pairs $(p$-value $<0.05)$ were retained. To 
811 simplify the GRN, we focused on the TFs-TFs network. As a result, several TFs

812 contained multiple genes, we combine those as TF modules, including TCPs, ARFs,

813 MYBs, and woxs.

\section{Data availability}

815 The raw sequence data were deposited in the Genome Sequence Archive

816 (https://bigd.big.ac.cn/gsa) under accession number xxx.

\section{Acknowledgements}

818 We thank Professor Falong Lu and Zhuo Du from IGDB-CAS for critical comments

819 on the manuscript. This research was supported by the Strategic Priority Research

820 Program of the Chinese Academy of Sciences (XDA24010204) and the National

821 Natural Sciences Foundation of China (31970631) to J.X.

\section{Author contributions}

823 J.X. and L.Z. designed and supervised the research and wrote the manuscript. H.Z.,

824 L.Z. and X.-L.L. performed Cut\&Tag, ATAC-seq and RNA-seq experiments; L.Z. 825 performed data analysis; X.-L.L., Y.-M.Y and H.W. performed the rest of the 826 experiments. All authors discussed the results and commented on the manuscript.

\section{Competing interests}

828 The authors declare no competing interests. 


\section{References}

830 1. Armenta-Medina, A. et al. Developmental and genomic architecture of plant 831 embryogenesis: from model plant to crops. Plant Commun 2, 100136 (2021).

832 2. Dresselhaus, T. \& Jurgens, G. Comparative Embryogenesis in Angiosperms: Activation and Patterning of Embryonic Cell Lineages. Annu Rev Plant Biol 72, 641-676 (2021).

3. Gerri, C., Menchero, S., Mahadevaiah, S.K., Turner, J.M.A. \& Niakan, K.K.

4. Xu, R., Li, C., Liu, X. \& Gao, S. Insights into epigenetic patterns in mammalian early embryos. Protein \& Cell 12, 7-28 \%U https://link.springer.com/10.1007/s13238-020-00757-z (2021).

5. Drost, H.G., Gabel, A., Grosse, I. \& Quint, M. Evidence for active maintenance

6. Quint, M. et al. A transcriptomic hourglass in plant embryogenesis. Nature 490,

7. Nodine, M.D. \& Bartel, D.P. Maternal and paternal genomes contribute equally

8. Zhao, P., Zhou, X., Zheng, Y., Ren, Y. \& Sun, M.-X. Equal parental contribution

9. Trevino, A.E. et al. Chromatin accessibility dynamics in a model of human forebrain development. Science 367, eaay1645 \%U https://www.sciencemag.org/lookup/doi/10.1126/science.aay1645 (2020).

10. Zhu, J. et al. Genome-wide chromatin state transitions associated with

11. Sharma, S., Kelly, T.K. \& Jones, P.A. Epigenetics in cancer. Carcinogenesis 31,

12. Baulcombe, D.C. \& Dean, C. Epigenetic regulation in plant responses to the environment. Cold Spring Harb Perspect Biol 6, a019471 (2014).

13. Cheng, Y. et al. Targeting epigenetic regulators for cancer therapy: mechanisms

862 14. Corces, M.R. et al. Lineage-specific and single-cell chromatin accessibility 
charts human hematopoiesis and leukemia evolution. Nat Genet 48, 1193-203 (2016).

15. Xia, W. et al. Resetting histone modifications during human parental-to-zygotic transition. Science 365, 353-360 (2019).

16. $\mathrm{Xu}, \mathrm{Q} . \& \mathrm{Xie}, \mathrm{W}$. Epigenome in Early Mammalian Development: Inheritance, Reprogramming and Establishment. Trends Cell Biol 28, 237-253 (2018).

17. Zhang, B. et al. Allelic reprogramming of the histone modification H3K4me3 in early mammalian development. Nature 537, 553-557 (2016).

18. Liu, X. et al. Distinct features of H3K4me3 and H3K27me3 chromatin domains in pre-implantation embryos. Nature 537, 558-562 (2016).

19. Zenk, F. et al. Germ line-inherited H3K27me3 restricts enhancer function

20. Huang, J.J. et al. Impairment of Preimplantation Porcine Embryo Development

21. Burton, A. \& Torres-Padilla, M.E. Chromatin dynamics in the regulation of cell

22. $\mathrm{Wu}, \mathrm{J}$. et al. The landscape of accessible chromatin in mammalian

23. Lu, F. et al. Establishing Chromatin Regulatory Landscape during Mouse 885

24. Radoeva, T. \& Weijers, D. A roadmap to embryo identity in plants. Trends Plant

25. Yi, F. et al. High Temporal-Resolution Transcriptome Landscape of Early

26. Radoeva, T. et al. A Robust Auxin Response Network Controls Embryo and

27. Xiang, D. et al. The Transcriptional Landscape of Polyploid Wheats and Their Diploid Ancestors during Embryogenesis and Grain Development. Plant Cell 31, 2888-2911 (2019). 
29. Kawakatsu, T., Nery, J.R., Castanon, R. \& Ecker, J.R. Dynamic DNA methylation reconfiguration during seed development and germination. Genome Biol 18, 171 (2017).

30. El Baidouri, M. et al. Reconciling the evolutionary origin of bread wheat (Triticum aestivum). New Phytol 213, 1477-1486 (2017).

31. Consortium, T.I.W.G.S. et al. Shifting the limits in wheat research and breeding using a fully annotated reference genome. Science 361(2018).

32. Vijverberg, K., Ozias-Akins, P. \& Schranz, M.E. Identifying and Engineering Genes for Parthenogenesis in Plants. Front Plant Sci 10, 128 (2019).

33. Kumar, V. \& Van Staden, J. New insights into plant somatic embryogenesis: an epigenetic view. Acta Physiologiae Plantarum 39, 1-17 (2017).

34. Buenrostro, J.D., Wu, B., Chang, H.Y. \& Greenleaf, W.J. ATAC-seq: A Method for Assaying Chromatin Accessibility Genome-Wide. Curr Protoc Mol Biol 109, 2129 1-21 299 (2015).

35. Kaya-Okur, H.S. et al. CUT\&Tag for efficient epigenomic profiling of small samples and single cells. Nat Commun 10, 1930 (2019).

36. Lu, Z. et al. The prevalence, evolution and chromatin signatures of plant regulatory elements. Nat Plants 5, 1250-1259 (2019).

37. Klemm, S.L., Shipony, Z. \& Greenleaf, W.J. Chromatin accessibility and the regulatory epigenome. Nat Rev Genet 20, 207-220 (2019).

38. Corces, M.R. et al. The chromatin accessibility landscape of primary human cancers. Science $\quad 362, \quad$ eaav1898 https://www.science.org/doi/10.1126/science.aav1898 (2018).

39. Giaimo, B.D., Ferrante, F., Herchenrother, A., Hake, S.B. \& Borggrefe, T. The histone variant H2A.Z in gene regulation. Epigenetics Chromatin 12, 37 (2019).

40. Ernst, J. \& Kellis, M. Chromatin-state discovery and genome annotation with ChromHMM. Nat Protoc 12, 2478-2492 (2017).

41. Ernst, J. \& Kellis, M. ChromHMM: automating chromatin-state discovery and characterization. Nat Methods 9, 215-6 (2012).

42. Gorkin, D.U. et al. An atlas of dynamic chromatin landscapes in mouse fetal development. Nature 583, 744-751 (2020).

43. Long, J. et al. Nurse cell--derived small RNAs define paternal epigenetic inheritance in Arabidopsis. Science 373, eabh0556 (2021).

44. Anderson, S.N. et al. The Zygotic Transition Is Initiated in Unicellular Plant Zygotes with Asymmetric Activation of Parental Genomes. Dev Cell 43, 
934 45. Schulz, K.N. \& Harrison, M.M. Mechanisms regulating zygotic genome activation. Nat Rev Genet 20, 221-234 (2019).

936

46. Xiao, J. et al. Cis and trans determinants of epigenetic silencing by Polycomb repressive complex 2 in Arabidopsis. Nat Genet 49, 1546-1552 (2017).

47. Bieluszewski, T., Xiao, J., Yang, Y. \& Wagner, D. PRC2 activity, recruitment, and silencing: a comparative perspective. Trends Plant Sci 26, 1186-1198 (2021).

48. Willemsen, V. \& Scheres, B. Mechanisms of pattern formation in plant embryogenesis. Annu Rev Genet 38, 587-614 (2004).

49. Horstman, A., Bemer, M. \& Boutilier, K. A transcriptional view on somatic embryogenesis. Regeneration (Oxf) 4, 201-216 (2017).

50. Wang, F.X. et al. Chromatin Accessibility Dynamics and a Hierarchical Transcriptional Regulatory Network Structure for Plant Somatic Embryogenesis. Dev Cell 54, 742-757 e8 (2020).

51. Su, Y.H., Tang, L.P., Zhao, X.Y. \& Zhang, X.S. Plant cell totipotency: Insights into cellular reprogramming. Journal of Integrative Plant Biology 63, 228-243 (2021).

52. Baud, S. \& Lepiniec, L. Physiological and developmental regulation of seed oil production. Prog Lipid Res 49, 235-49 (2010).

53. Skubacz, A., Daszkowska-Golec, A. \& Szarejko, I. The Role and Regulation of ABI5 (ABA-Insensitive 5) in Plant Development, Abiotic Stress Responses and Phytohormone Crosstalk. Front Plant Sci 7, 1884 (2016).

54. Ikeuchi, M., Ogawa, Y., Iwase, A. \& Sugimoto, K. Plant regeneration: cellular origins and molecular mechanisms. Development 143, 1442-51 (2016).

55. Ramirez-Gonzalez, R.H. et al. The transcriptional landscape of polyploid wheat. Science $\quad 361$ eaar6089 \%U https:/www.sciencemag.org/lookup/doi/10.1126/science.aar6089 (2018).

56. Simonini, S. et al. The Polycomb group protein MEDEA controls cell proliferation and embryonic patterning in Arabidopsis. Dev Cell 56, 1945-1960 e7 (2021).

57. Armenta-Medina, A. et al. Developmental and genomic architecture of plant embryogenesis: from model plant to crops. Plant Communications 2, 100136 (2021).

58. Chen, S., Zhou, Y., Chen, Y. \& Gu, J. fastp: an ultra-fast all-in-one FASTQ 
preprocessor. Bioinformatics 34, i884-i890 (2018).

969

970

971

972

973

974

975

976

977

978

979

980

981

982

983

984

985

986

987

988

989

990

991

992

993

994

995

996

997

998

999

1000

1001

1002

59. Li, H. \& Durbin, R. Fast and accurate short read alignment with Burrows-Wheeler transform. Bioinformatics 25, 1754-60 (2009).

60. Kim, D., Paggi, J.M., Park, C., Bennett, C. \& Salzberg, S.L. Graph-based genome alignment and genotyping with HISAT2 and HISAT-genotype. Nat Biotechnol 37, 907-915 (2019).

61. Liao, Y., Smyth, G.K. \& Shi, W. featureCounts: an efficient general purpose program for assigning sequence reads to genomic features. Bioinformatics $\mathbf{3 0}$, 923-30 (2014).

62. Robinson, M.D., McCarthy, D.J. \& Smyth, G.K. edgeR: a Bioconductor package for differential expression analysis of digital gene expression data. Bioinformatics 26, 139-40 (2010).

63. Langfelder, P. \& Horvath, S. WGCNA: an R package for weighted correlation network analysis. BMC Bioinformatics 9, 559 (2008).

64. Yu, G.C., Wang, L.G., Han, Y.Y. \& He, Q.Y. clusterProfiler: an R Package for Comparing Biological Themes Among Gene Clusters. Omics-a Journal of Integrative Biology 16, 284-287 (2012).

65. Zheng, Y. CUT\&Tag Data Processing and Analysis Tutorial. (2020).

66. Ramirez, F., Dundar, F., Diehl, S., Gruning, B.A. \& Manke, T. deepTools: a flexible platform for exploring deep-sequencing data. Nucleic Acids Res 42, W187-91 (2014).

67. Jin, H.J. et al. ChIPseqSpikeInFree: a ChIP-seq normalization approach to reveal global changes in histone modifications without spike-in. Bioinformatics 36, 1270-1272 (2020).

68. Meers, M.P., Tenenbaum, D. \& Henikoff, S. Peak calling by Sparse Enrichment Analysis for CUT\&RUN chromatin profiling. Epigenetics Chromatin 12, 42 (2019).

69. Zhang, Y. et al. Model-based analysis of ChIP-Seq (MACS). Genome Biol 9, R137 (2008).

70. Quinlan, A.R. \& Hall, I.M. BEDTools: a flexible suite of utilities for comparing genomic features. Bioinformatics 26, 841-2 (2010).

71. Yu, G., Wang, L.G. \& He, Q.Y. ChIPseeker: an R/Bioconductor package for ChIP peak annotation, comparison and visualization. Bioinformatics 31, 2382-3 (2015).

72. Love, M.I., Huber, W. \& Anders, S. Moderated estimation of fold change and 
73. Gusmao, E.G., Dieterich, C., Zenke, M. \& Costa, I.G. Detection of active transcription factor binding sites with the combination of DNase hypersensitivity and histone modifications. Bioinformatics 30, 3143-51 (2014).

74. Khan, A. et al. JASPAR 2018: update of the open-access database of transcription factor binding profiles and its web framework. Nucleic acids research 46, D260-D266 (2018).

75. Heinz, S. et al. Simple combinations of lineage-determining transcription factors prime cis-regulatory elements required for macrophage and B cell identities. Mol Cell 38, 576-89 (2010).

1013 76. Meinke, D.W. Genome-wide identification of EMBRYO-DEFECTIVE (EMB) genes required for growth and development in Arabidopsis. New Phytol 226, 306-325 (2020).

77. Buchfink, B., Xie, C. \& Huson, D.H. Fast and sensitive protein alignment using DIAMOND. Nature Methods 12, 59-60 (2015).

78. Buchfink, B., Reuter, K. \& Drost, H.G. Sensitive protein alignments at tree-of-life scale using DIAMOND. Nature Methods 18, 366-+ (2021).

79. Hao, Z. et al. Conserved, divergent and heterochronic gene expression during Brachypodium and Arabidopsis embryo development. Plant Reprod 34, 207-224 (2021).

80. Leiboff, S. \& Hake, S. Reconstructing the Transcriptional Ontogeny of Maize and Sorghum Supports an Inverse Hourglass Model of Inflorescence Development. Curr Biol 29, 3410-3419 e3 (2019).

81. Levin, M. et al. The mid-developmental transition and the evolution of animal body plans. Nature 531, 637-641 (2016).

1028 82. Gu, Z., Eils, R. \& Schlesner, M. Complex heatmaps reveal patterns and correlations in multidimensional genomic data. Bioinformatics 32, 2847-9 (2016). 
a
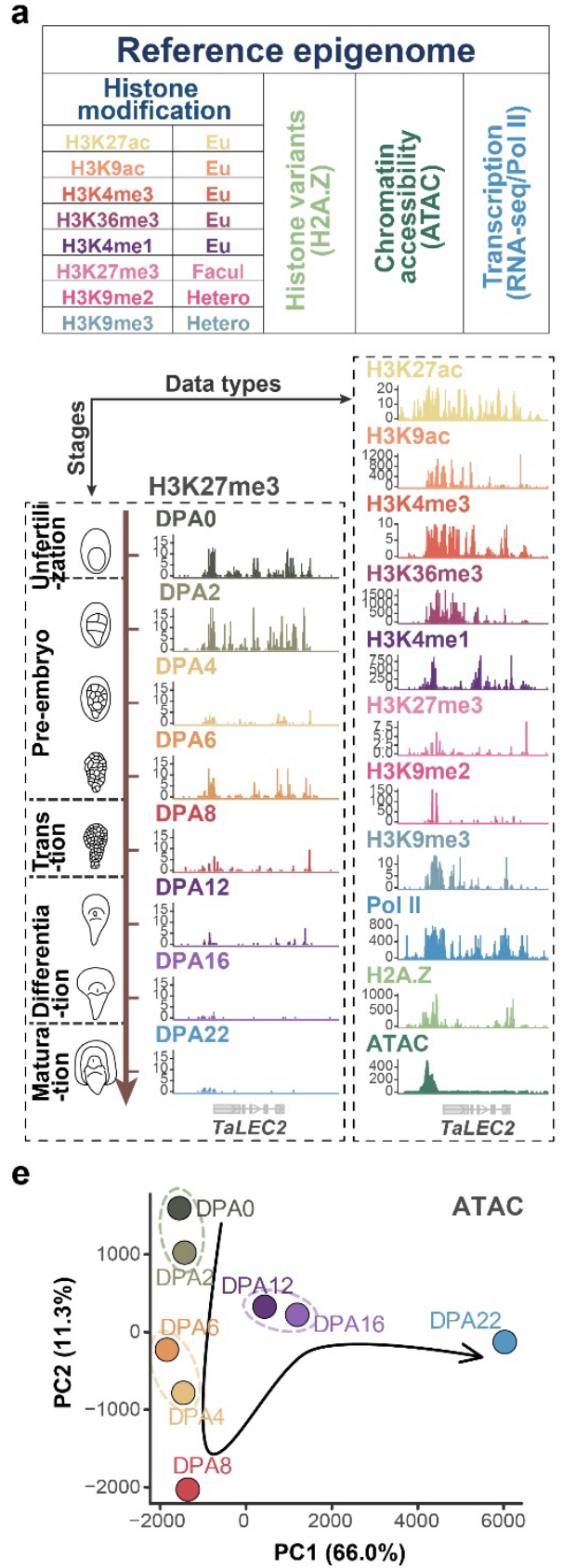

b
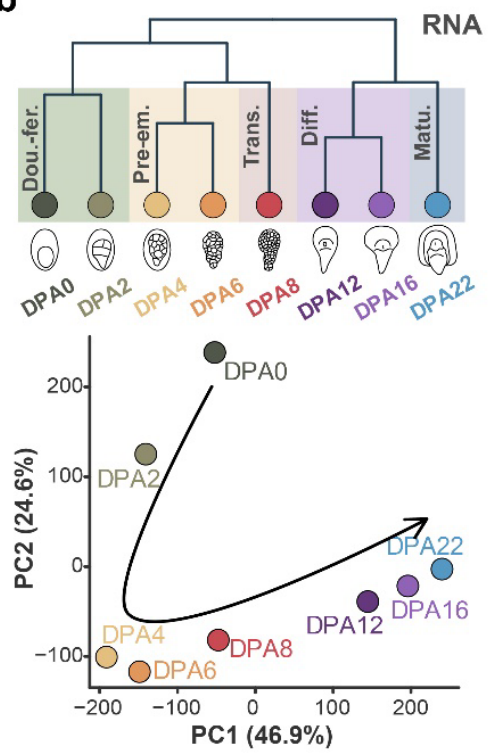

d Embryonic development process

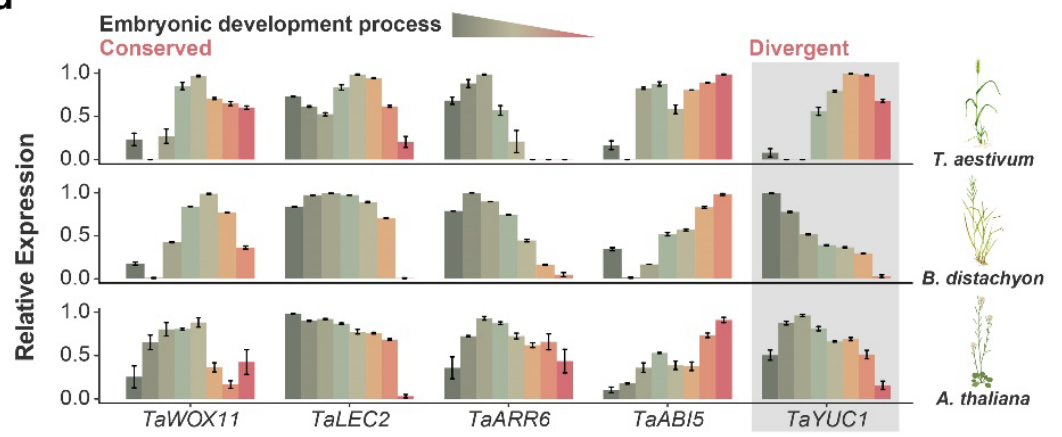

f

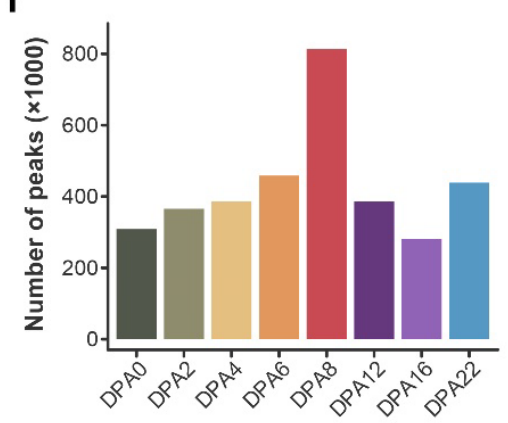

C

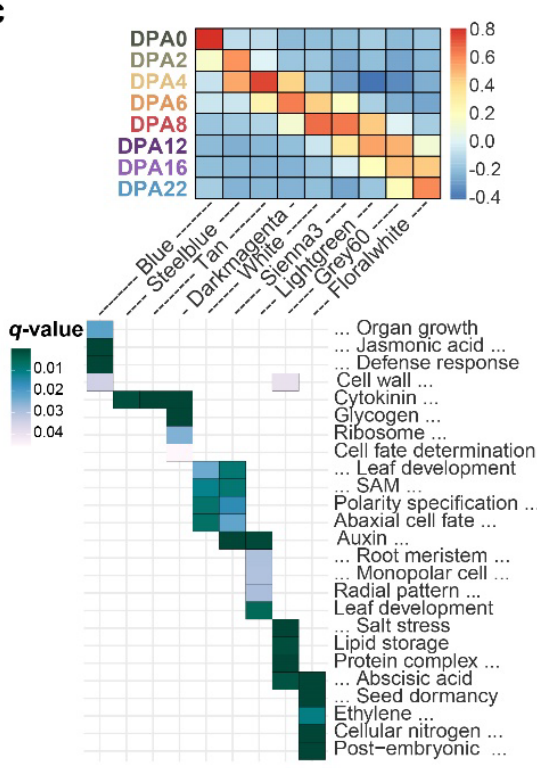

g

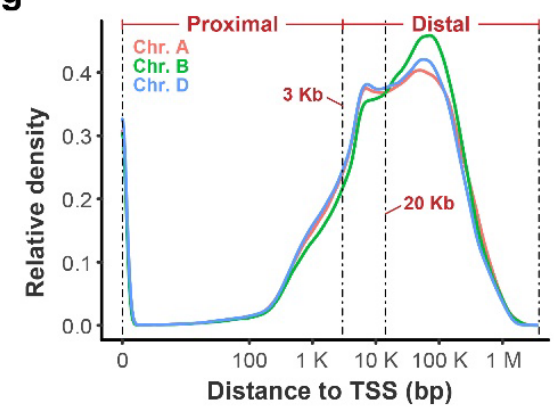

Fig. 1 | Charting chromatin landscapes of wheat embryogenesis

a, Experimental design and major axes of the data series: data types and sampling developmental stages, DPA: days post anthesis.

b, Cluster dendrogram and PCA of transcriptome showing five distinct development stages: around double fertilization (DPA 0/2), pre-embryo (DPA 4/6), transition (DPA 8), differentiation (DPA 12/16), and maturation (DPA 22).

c, Representative modules from WGCNA cluster and enrichment of corresponding GO.

d, The conserved and diversified expression pattern of representative genes between monocot ( $T$. aestivum and B. distachyon) and dicot (A. thaliana) during embryogenesis.

e-g, Dynamic pattern of genome-wide chromatin accessibility measured by ATACseq during embryogenesis. PCA analysis (e), peak numbers dynamics (f), and peak distribution in different sub-genomes (g). 
bioRxiv preprint doi: https://doi.org/10.1101/2022.01.21.477188; this version posted January 23, 2022. The copyright holder for this preprint (which was not certified by peer review) is the author/funder. All rights reserved. No reuse allowed without permission.

a

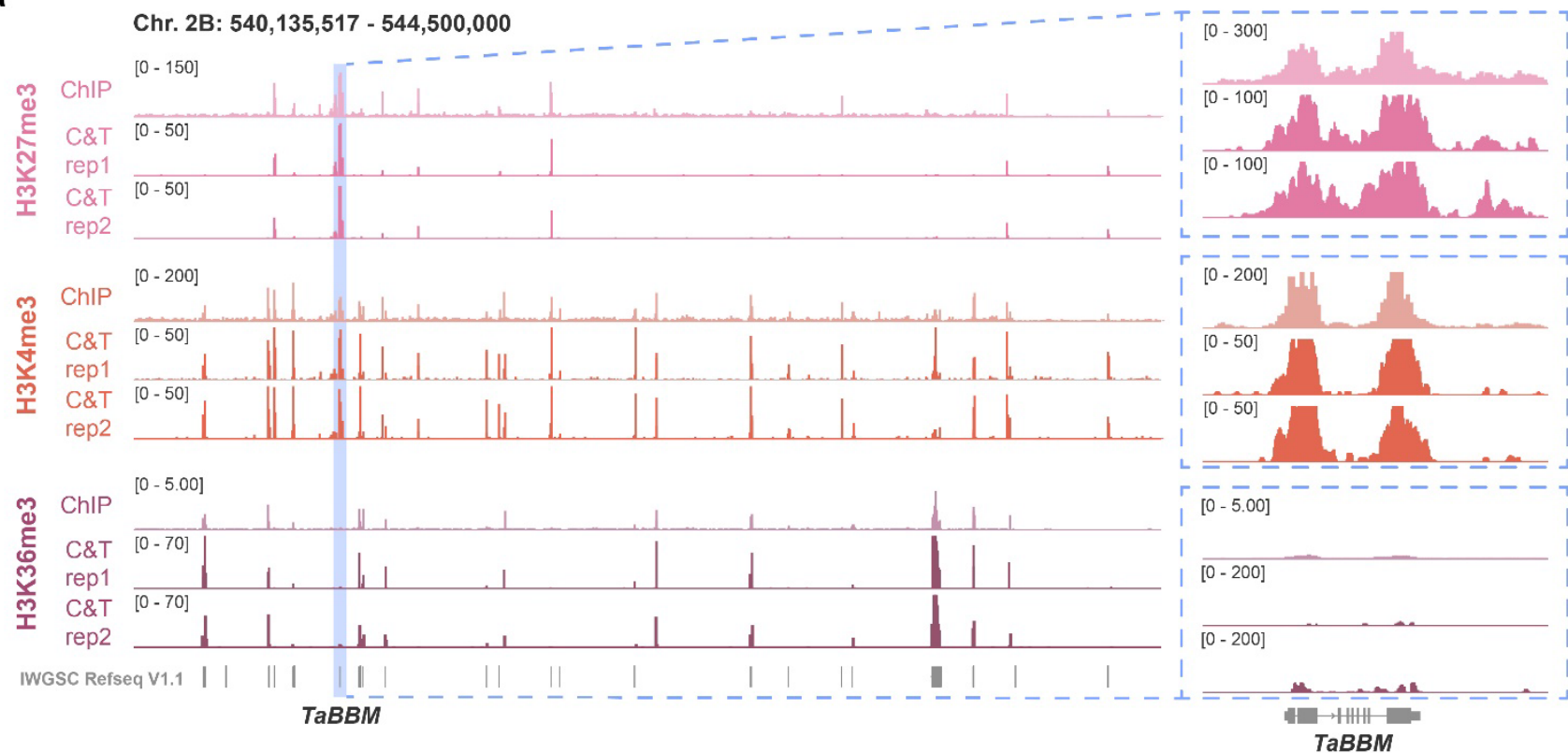

b

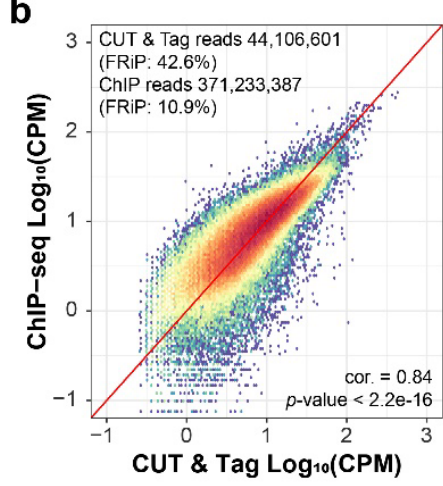

d

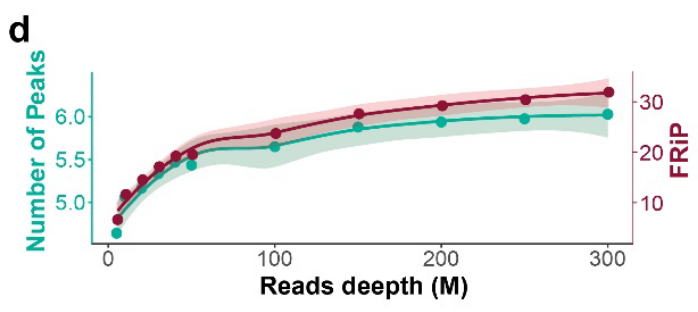

e

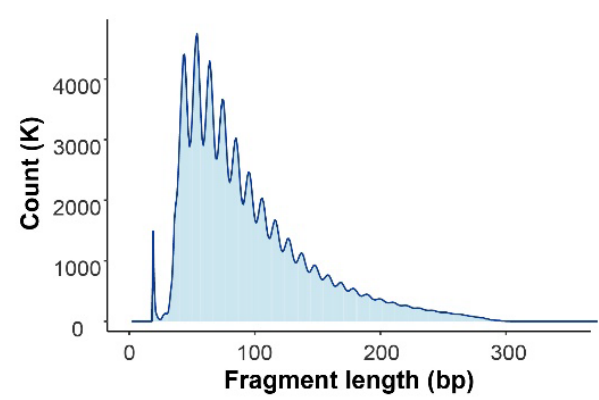

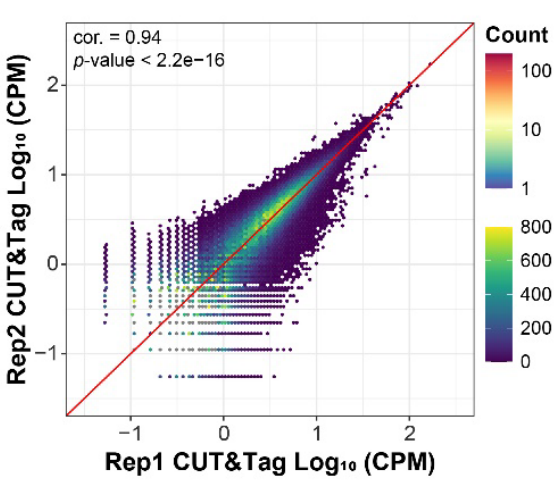

f

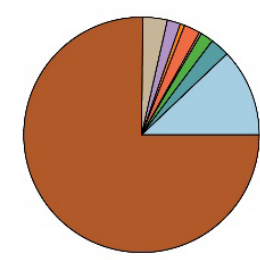

h

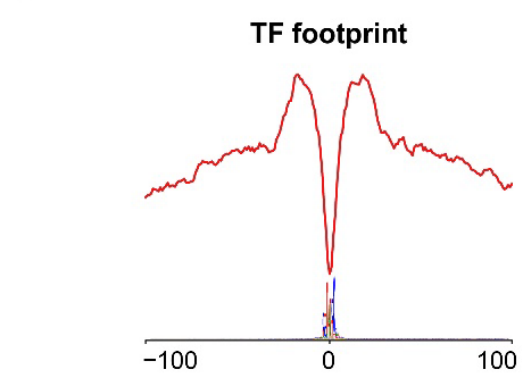

ATAC peaks distribution

$\square$ Promoter $(<=1 \mathrm{~kb})(12.08 \%)$

$\square$ Promoter $(1-2 \mathrm{~kb})(2.66 \%)$

$\square$ Promoter (2-3kb) $(1.76 \%$

1 st Exon $(0.31 \%)$

$\square$ Other Exon (2.22\%)

1st Intron $(0.61 \%)$

Other Intron (1.83\%)

Downstream $(<=300)(3.35 \%)$

Distal Intergenic $(75.18 \%)$
Cor p-value Reads ChIP Reads C\&T

$\begin{array}{lllll}\text { H3K4me3 } & 0.85 & 2.2 \mathrm{E}-16 & 30,794,663 & 10,546,669\end{array}$

H3K36me3 $0.82 \quad 2.2 \mathrm{E}-16 \quad 78,041,981 \quad 26,762,579$

$\begin{array}{lllll}\text { H3K9ac } & 0.81 & 2.2 \mathrm{E}-16 & 647,729,900 & 29,951,717\end{array}$

H3K27me3 $\quad 0.84 \quad 2.2 E-16 \quad 371,233,387 \quad 44,106,601$

\section{g}

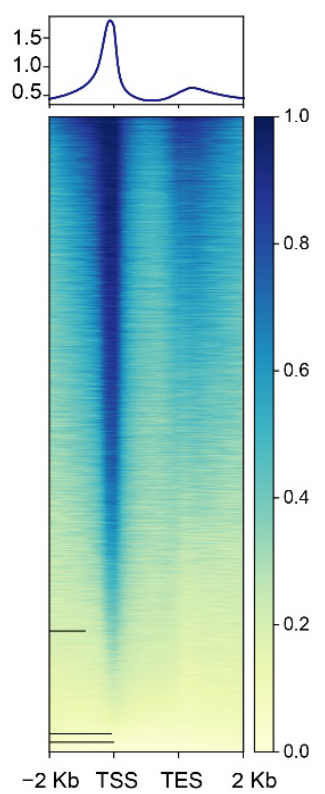

$$
\begin{array}{r}
7 \\
1 \\
1 \\
1 \\
1 \\
1 \\
1 \\
1 \\
-7 \\
1 \\
1 \\
1 \\
1 \\
1 \\
1 \\
=1 \\
7 \\
1 \\
1 \\
1 \\
1 \\
1 \\
1 \\
= \\
1
\end{array}
$$




\section{Fig. S1 | Quality control for CUT \& Tag and ATAC-seq}

a-c, CUT \& Tag recapture ChIPseq signal with high reproducibility and low sequencing depth. IGV (Integrated Genome Browser) showing high reproducibility between ChIP-seq and CUT \& Tag in seedlings (a). Correlation between ChIP-seq and CUT \& Tag as well as two biological replicates (b). Sequencing depth comparison for various histone modifications between CUT\&Tag and ChIPseq (c).

d-h, Quality control of ATAC-seq data. Peaks number, and FRiP (The fraction of reads in called peak regions) were calculated at different sequence depths (d). Fragment size distribution (e) and peak distribution within the genome (f). ATAC-seq signal distribution along with genes (g). Transcription factor footprint profile of ATAC-seq data $(\mathbf{h})$. 
bioRxiv preprint doi: https://doi.org/10.1101/2022 01 21.477188; this version posted January 23, 2022. The copyright holder for this preprint (which was not certified by peer review) is the author/funder. All rights reserved. No reuse allowed without permission.

a

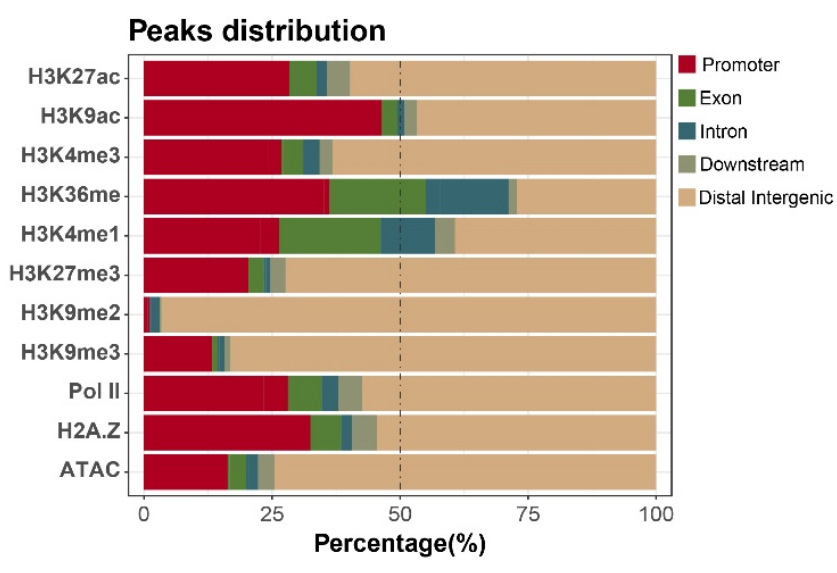

b

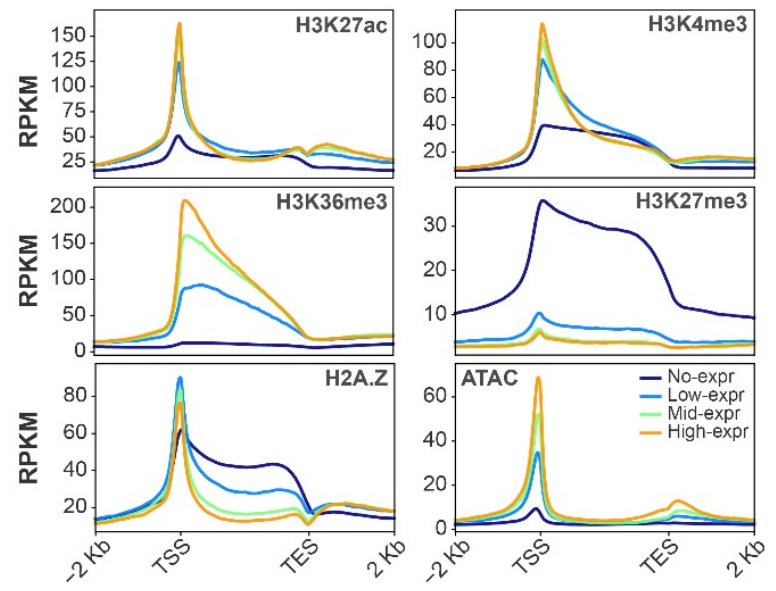

C

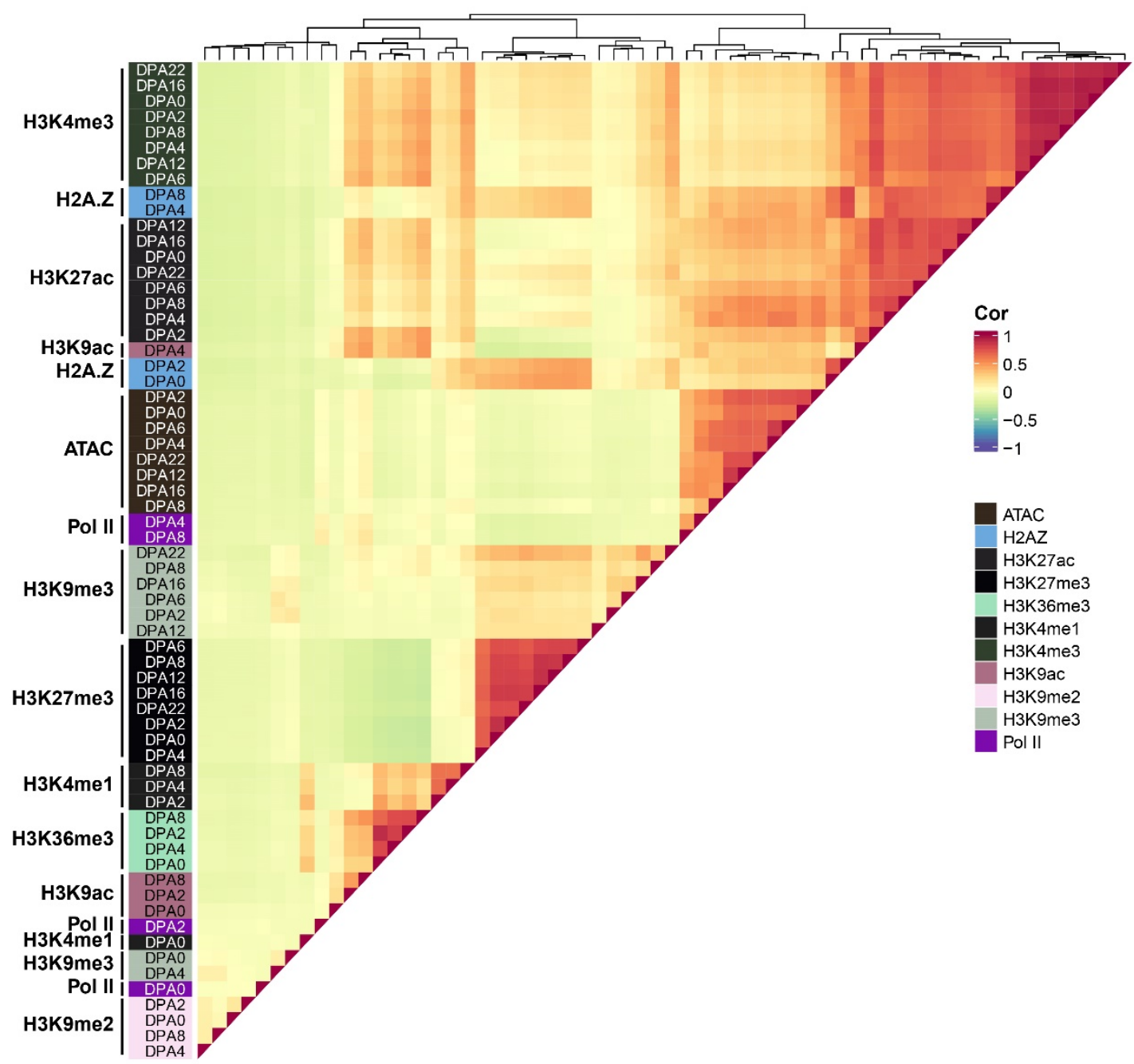

d

H3K27ac

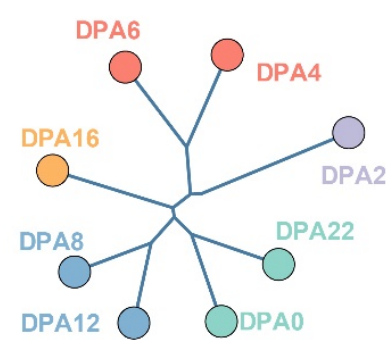

H3K4me3

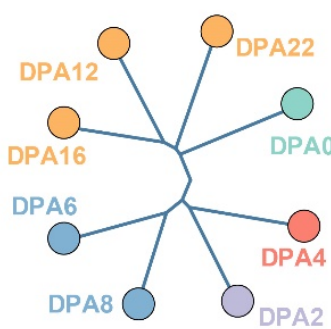

H3K27me3

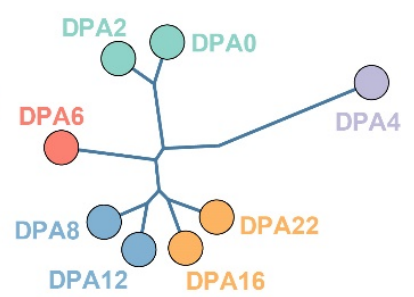

H3K9me3

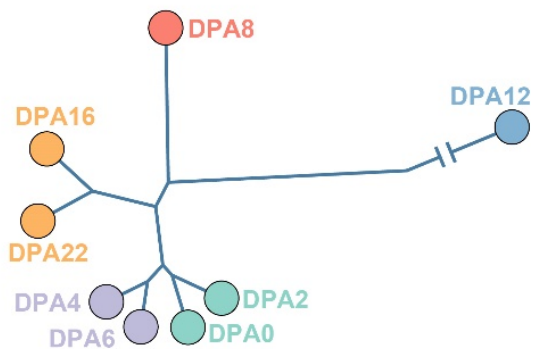




\section{Fig. S2 | Features of various histone modifications}

a, Peak distribution of different histone modifications relative to genes.

b, Correlation between different types of histone modification profiles and gene expression levels.

c, Pair-wise correlation map among different histone modification profiles. Jaccard index was calculated based on the peaks overlap, and then Pearson correlation scores were generated.

d, Cluster dendrogram of histone modifications. Reads count in peaks were normalized by CPM, and euclidean distances were calculated for tree clustering. 
bioRxiv preprint doi: https://doi org/10.1101/2022.01.21.477188; this version posted January 23, 2022. The copyright holder for this preprint (which was not certified by peer review) is the author/funder. All rights reserved. No reuse allowed without permission.

a

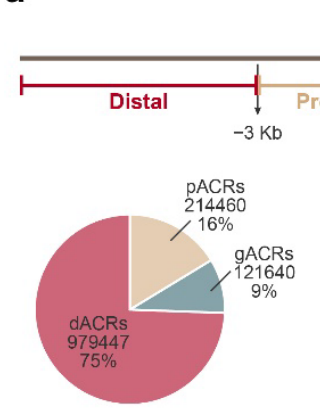

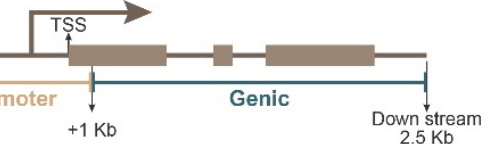

dACRs $(F C=5.4) \quad \operatorname{pACRs}(F C=4.6) \quad \operatorname{gACRs}(F C=2.2)$
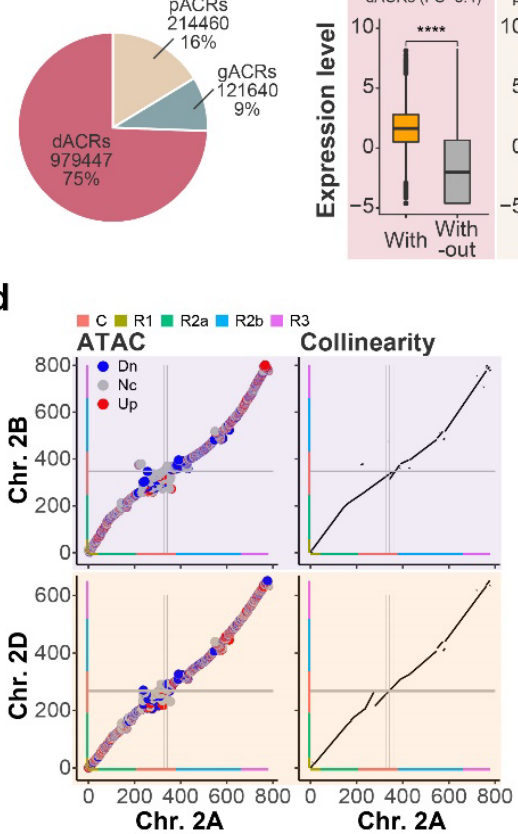

g

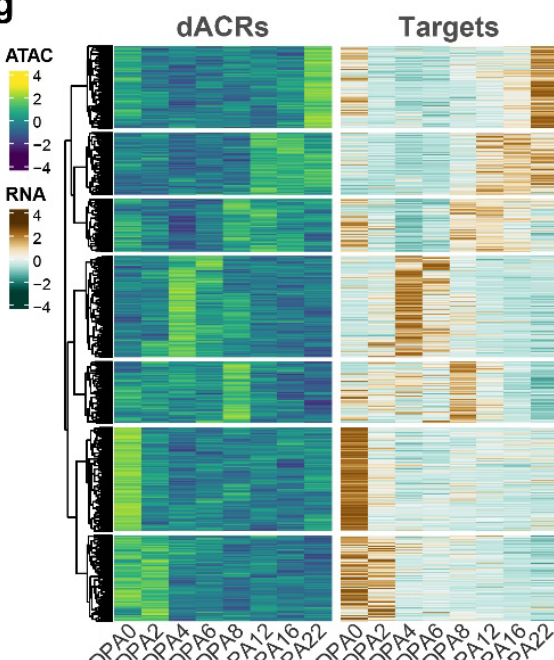

e

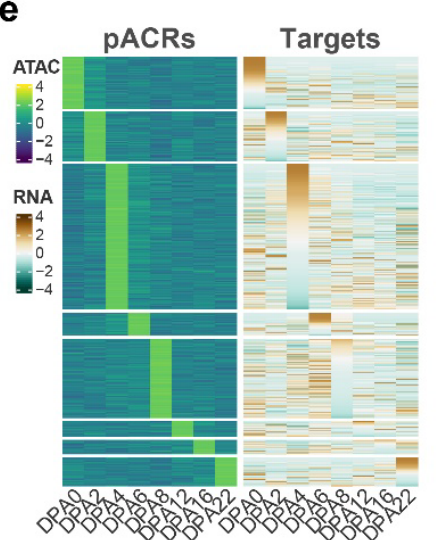

b

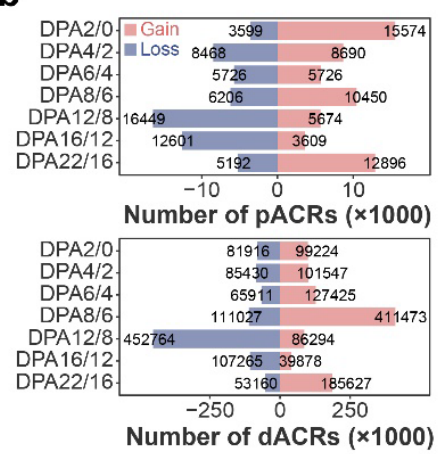

C

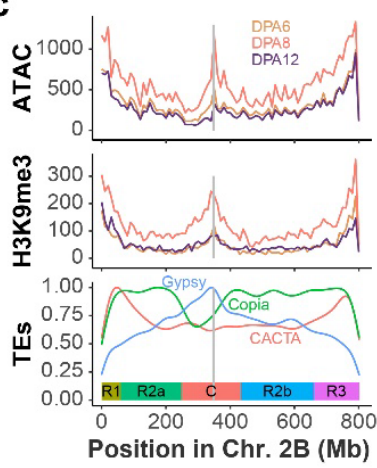

$\mathbf{f}$

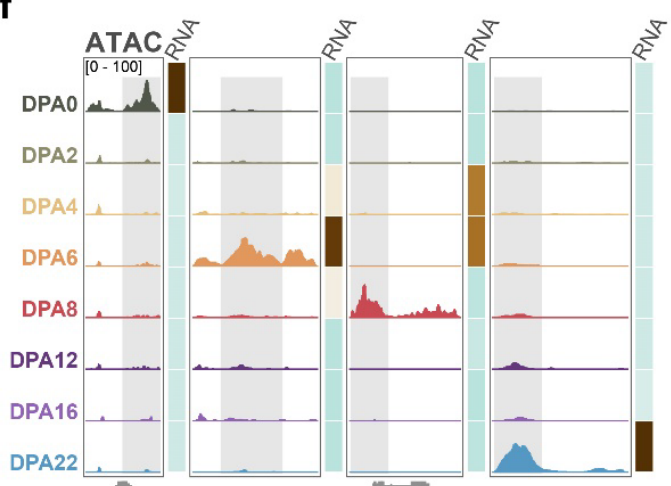

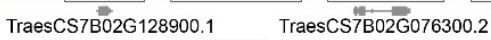

TraescS5 $502 \mathrm{G} 464500.1$

TraesCS4A02G373000.1

h

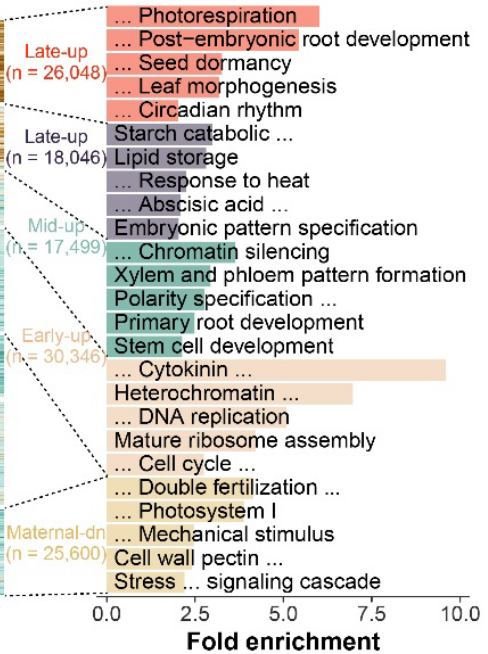

Target genes 43,901

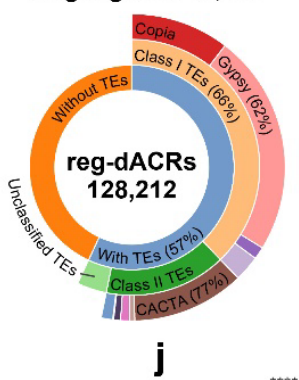

Class I TEs

- Copia

In Gypsy
- LINE

$=$ Unclassed LTR

Class II TES

- CACTA

II Harbinge
Mariner

- Mutato

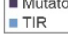

i

j

k
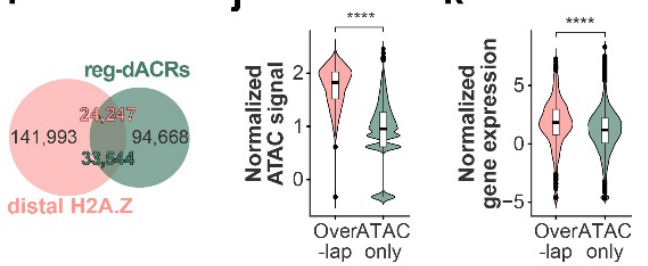


\section{Fig. 2 | Distinct feature of different ACRs in transcriptional regulation in wheat}

a, Subdividing ACRs based on distribution pattern around genes, and correlation between different types of ACRs and genes expression.

b, Distinct gain and loss of pACRs and dACRs between adjacent embryonic developmental stages. c, ATAC peaks, H3K9me3 and various types of TEs distributions on chromosome $2 \mathrm{~B}$ at different embryogenesis stages.

d, Comparisons of chromatin accessibility of collinear regions among subgenomes, chromosome $2 \mathrm{~B}$ compared to 2A (up-left) and chromosome 2D with 2A (down-left). Collinear regions were identified based on sequence conservation (right). Color bars on $\mathrm{x}$ and $\mathrm{y}$ axis indicate the chromosome segments defined by Consortium (IWGSC) et al., 2018. Grey lines indicate the centromere for individual chromosomes.

e, Stage-specific pACRs and corresponding genes expression.

f, Representative genes in e.

g, Heatmap showing correlation between reg-dACRs and corresponding genes (left) and GO enrichment for different genes sets that were clustered based on the linking reg-dACRs dynamic (right).

h, Fraction of reg-dACRs overlapped with various types of TEs.

i, Overlap between reg-dACRs and distal H2A.Z histone variant.

$\mathbf{j}$ and $\mathbf{k}$, Comparisons of ATAC signal intensity (j) and targets expression (k) between reg-dACRs overlapped with H2A.Z and those not overlapped. Mann-Whitney U test (two-sided) was used for a, j and k. $*: \mathrm{p}<=0.05 ; * *: \mathrm{p}<=0.01 ; * * *: \mathrm{p}<=0.001 ; * * * *: \mathrm{p}<=0.0001$. 
a

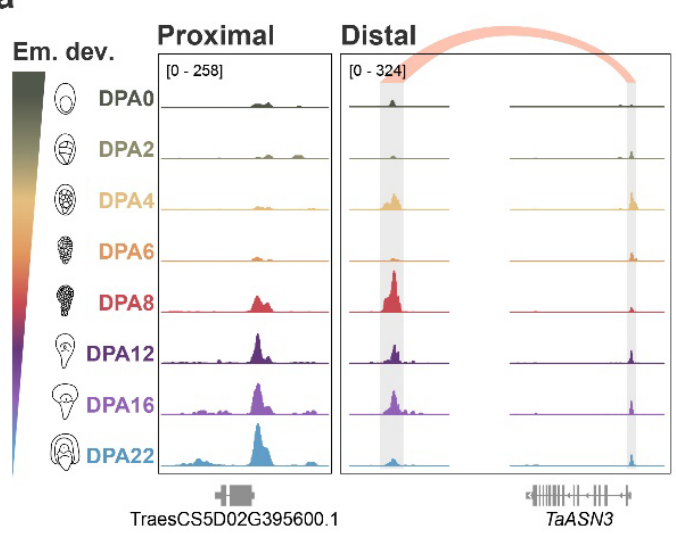

C
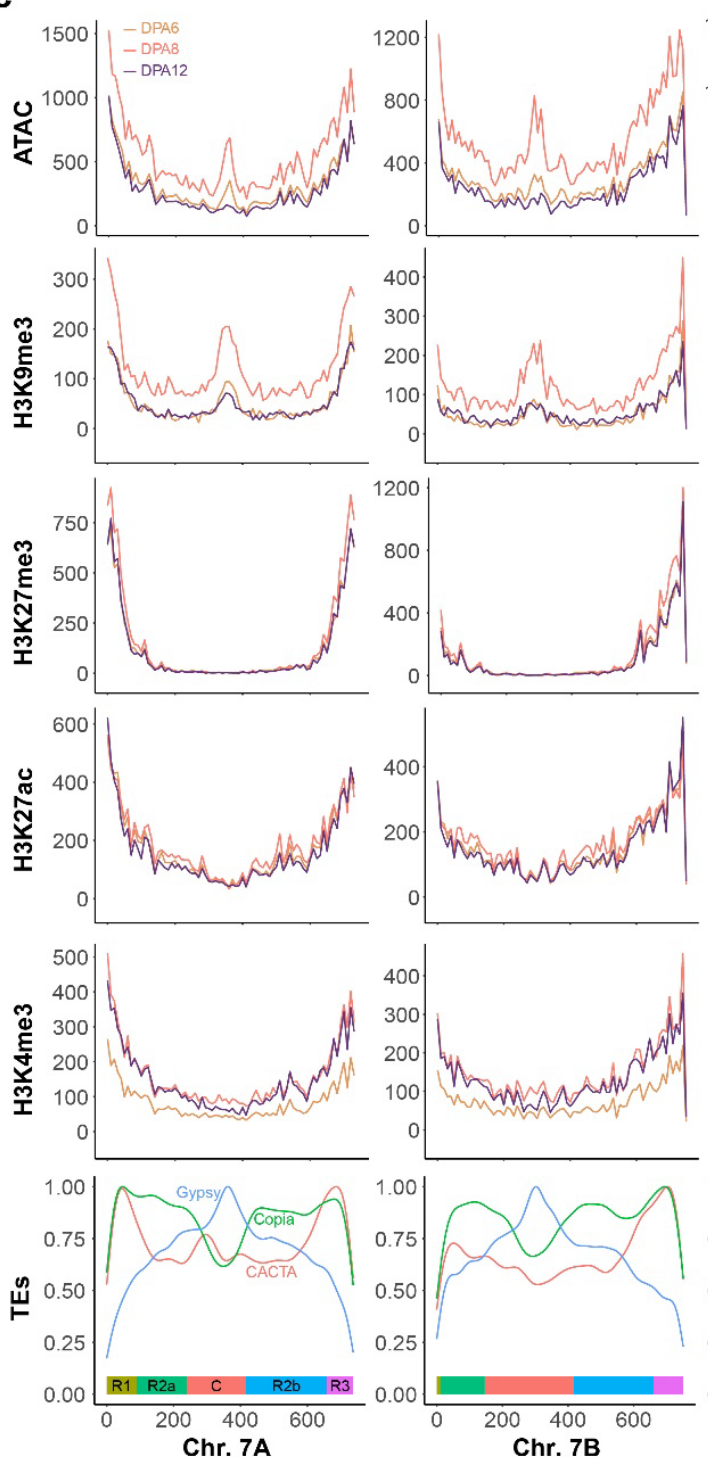

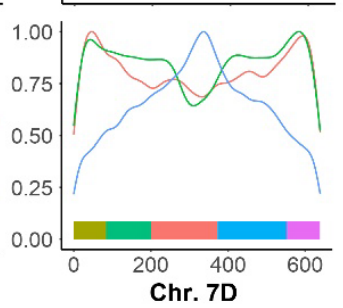

b

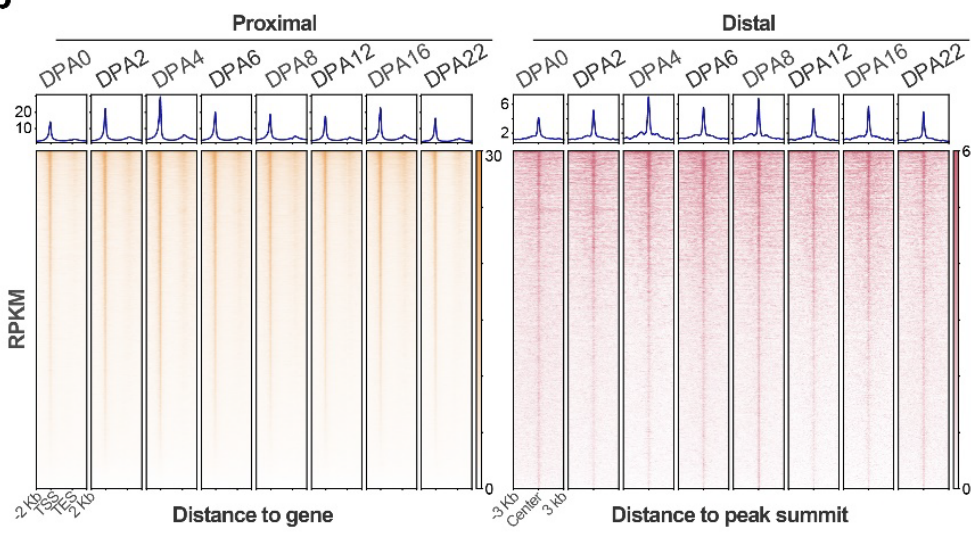

d
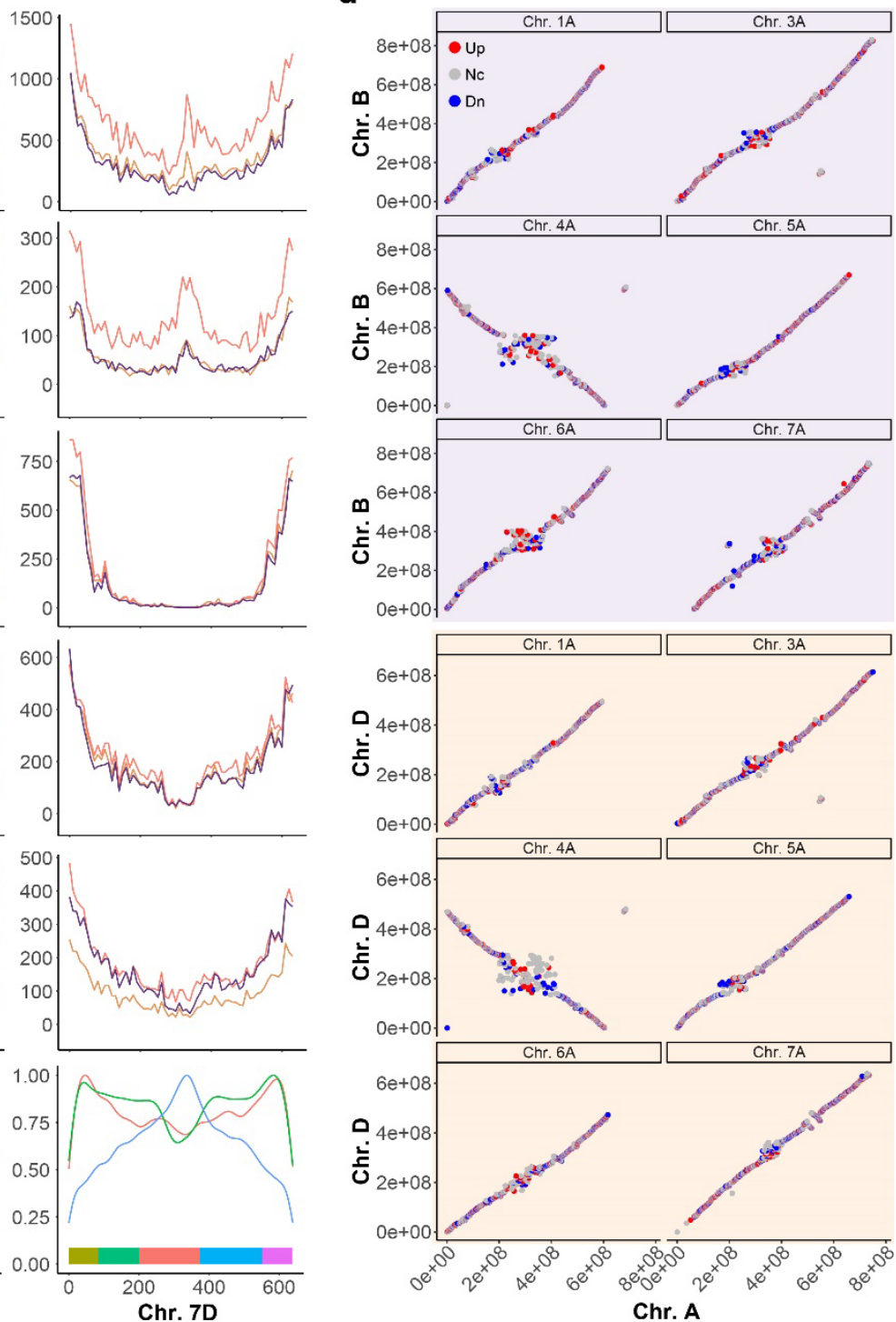
Fig. S3 | Dynamic ACRs during wheat embryogenesis and difference among sub-genomes a, IGV showing chromatin accessibility change cross wheat embryogenesis in both proximal and distal regions.

b, ACRs dynamic in proximal (left) and distal regions (right) during wheat embryogenesis.

c, Chromatin accessibility (ATAC), histone modifications and various types of TEs distributions change on Chr. 7 of A-, B- and D-subgenome at DPA 6, 8 and 12. Color bars on $\mathrm{x}$ and $\mathrm{y}$ axis indicate the chromosome segments defined by Consortium (IWGSC) et al., 2018.

d, Comparisons of chromatin accessibility of collinear regions among subgenomes. Down- (Dn), and Up-regulated (Up) loci were define by the $\log _{2}$ (fold change Chr. B/Chr. A) (left top-panel) and $\log _{2}$ (fold change Chr. D/Chr. A) (left bottom-panel) values bigger and lower than 1 and -1 . Nc indicates no change between subgenomes. 
bioRxiv preprint doi: https://doi.org/10.1101/2022.01.21.477188; this version posted January 23, 2022. The copyright holder for this preprint (which was not certified by peer review) is the author/funder. All rights reserved. No reuse allowed without permission.

a

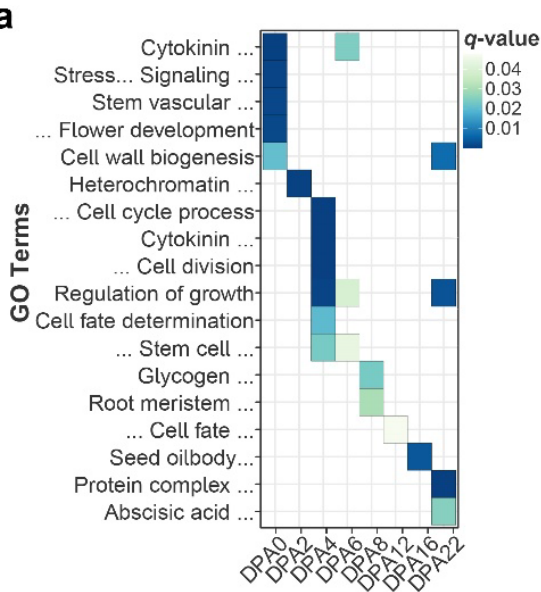

C

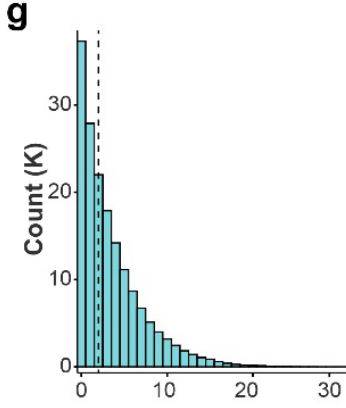

Number of genes skipped

$\mathbf{k}$

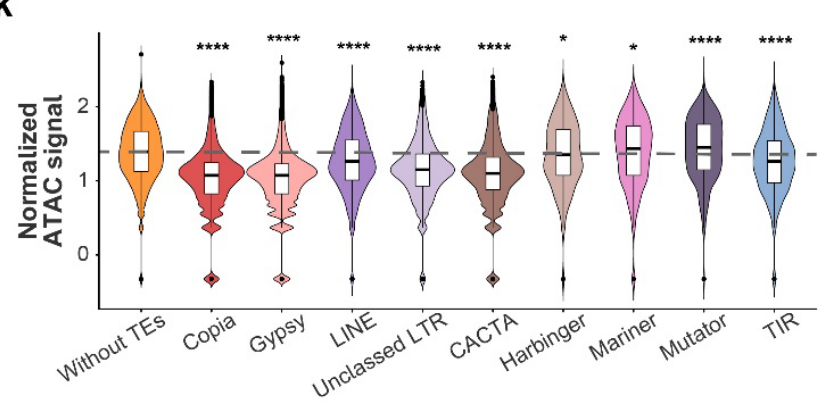

b

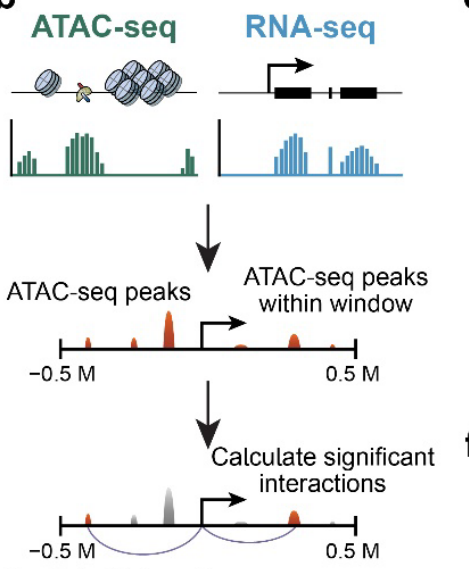

Predicted interactions
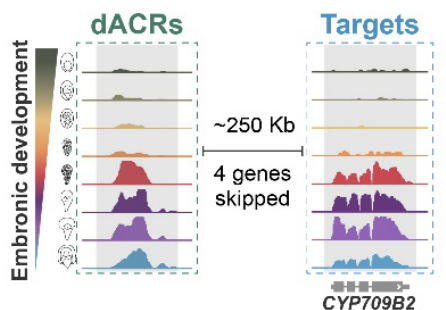

h

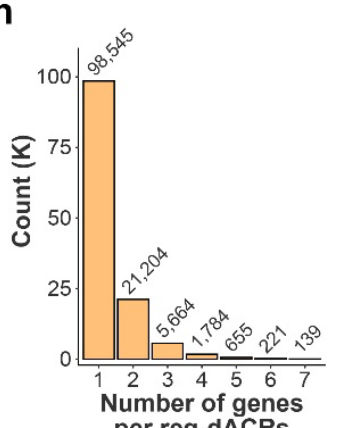

i d

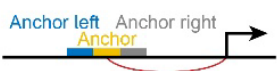

$\mathrm{Hi}-\mathrm{C}$ interaction

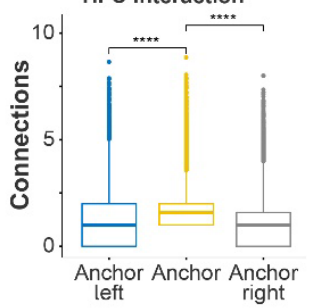

f

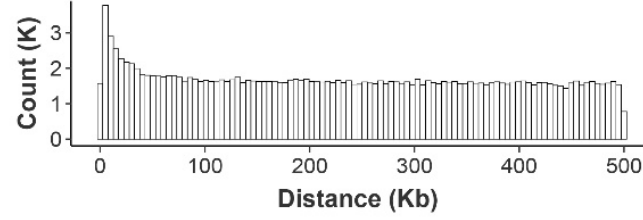

j

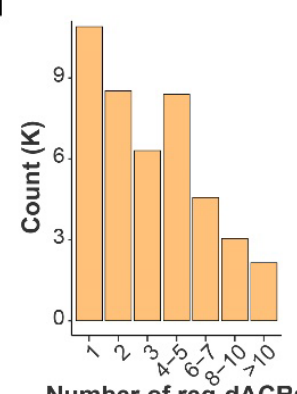

Number of reg-dACR per gene

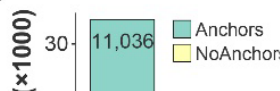

e

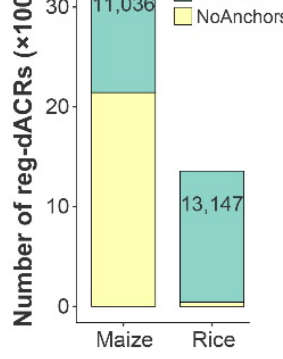

... Chromosome organization

Cytokine production

Maintenance of DNA methylation

Gametophyte development

Stress-activated protein ...

Primary root development

Cellular response to stress

है Regulation of seed germination

Starch metabolic process

Histone phosphorylation

Macromolecule modification

... Catalytic activity

Regulation of signal transduction

Purine nucleotide biosynthetic process

Cell morphogenesis

... GTPase ... Signal transduction

... Compound metabolic process

Tricarboxylic acid cycle

Carbohydrate phosphorylation

Mitotic G2 phase

l

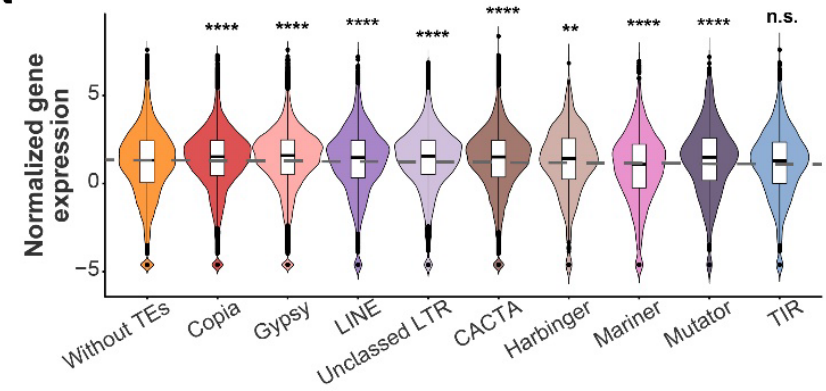




\section{Fig. S4 | Association between reg-dACRs to genes}

a, GO enrichment of stage-specific genes with pACRs.

b, Schematic of the strategy used to link distal ATAC-seq peaks to genes (see Method for detail).

c, Relative variation of expression level for genes with dACRs or without.

d, Hi-C anchors highlight the connection between reg-dACRs and target genes.

e, Number of dACRs homologous segment in maize and rice. Based on previously published Hi-C and ChIA-pet data, the homologous segment was grouped in anchors and NoAnchors.

$\mathbf{f}, \mathbf{g}$, Distance of reg-dACR to genes (f) and the number of genes skipped (g) between reg-dACR and target genes.

h, Number of genes can be targeted per reg-dACRs.

i, Number of reg-dACRs distribution for the individual target gene.

j, Corresponding GO enrichment for genes with solo or multiple dACRs.

$\mathbf{k}, \mathbf{l}$, Comparison of ATAC-seq signal and corresponding target genes expression among reg-dACRs overlapped by different types of TEs. Mann-Whitney U test (two-sided) was used for $\mathbf{c}$ and $\mathbf{k}(*: \mathrm{p}$ $<=0.05 ; * *: \mathrm{p}<=0.01 ; * * *: \mathrm{p}<=0.001)$. 
bioRxiv preprint doi: https://doi.org/10.1101/2022 01 21.477188; this version posted January 23, 2022. The copyright holder for this preprint (which was not certified by peer review) is the author/funder. All rights reserved. No reuse allowed without permission.

a

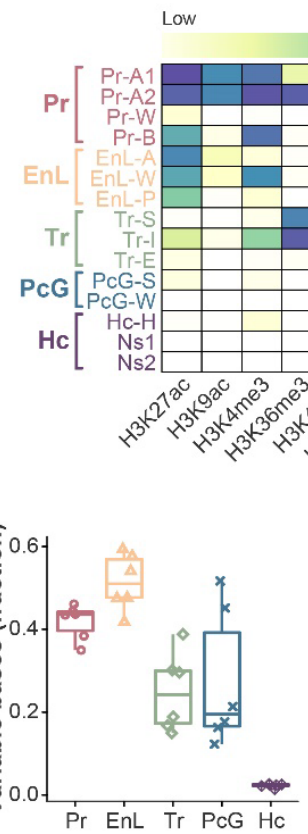

d

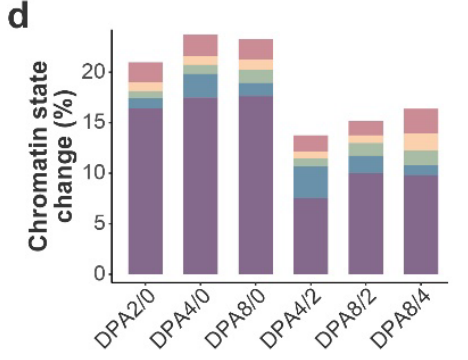

h

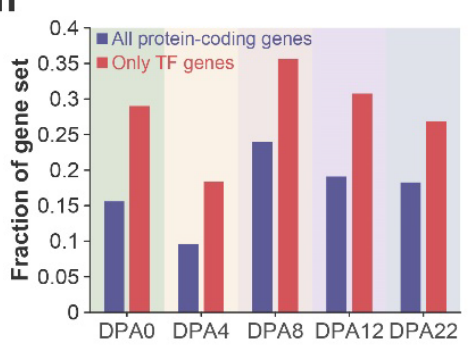

High Low

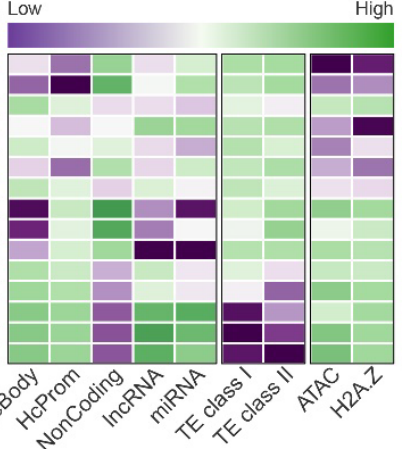

High

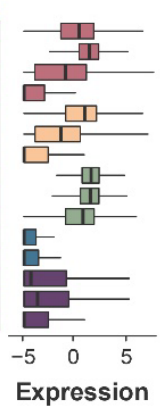

b

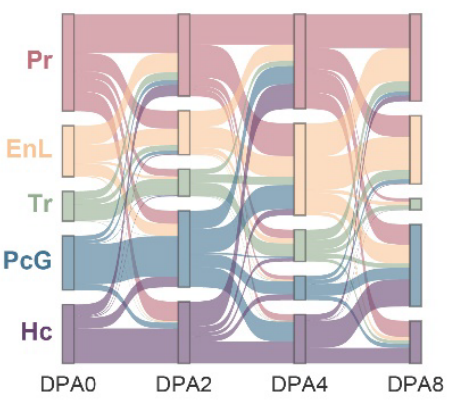

e H3K27ac

H3K4me3 H3K27me3

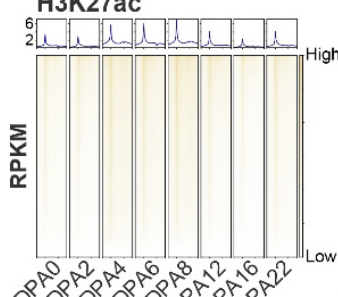

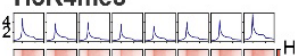

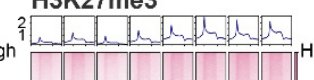

H3K9me3

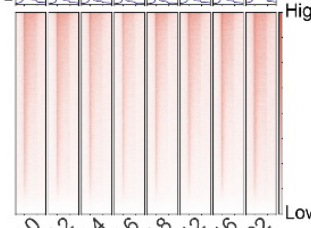

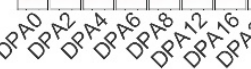

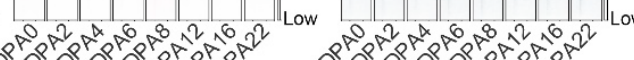

f

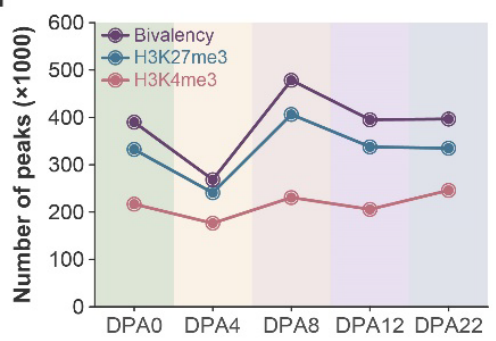

i

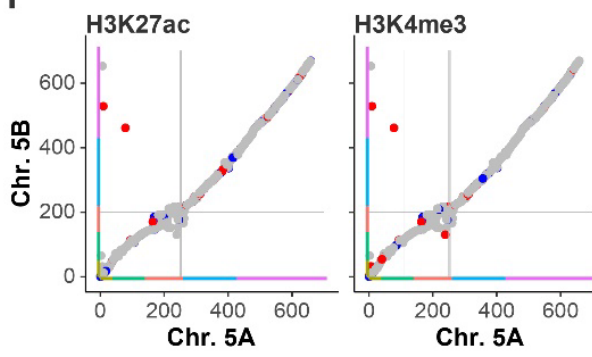

g

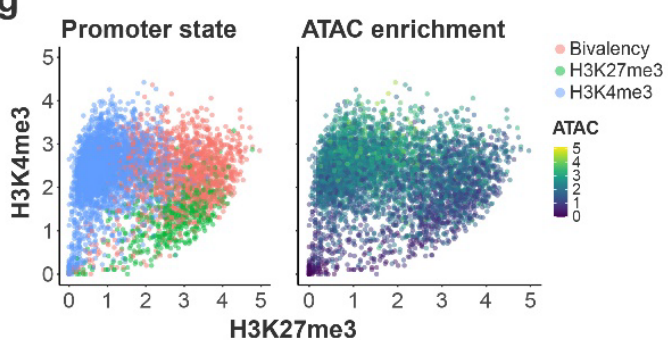

j

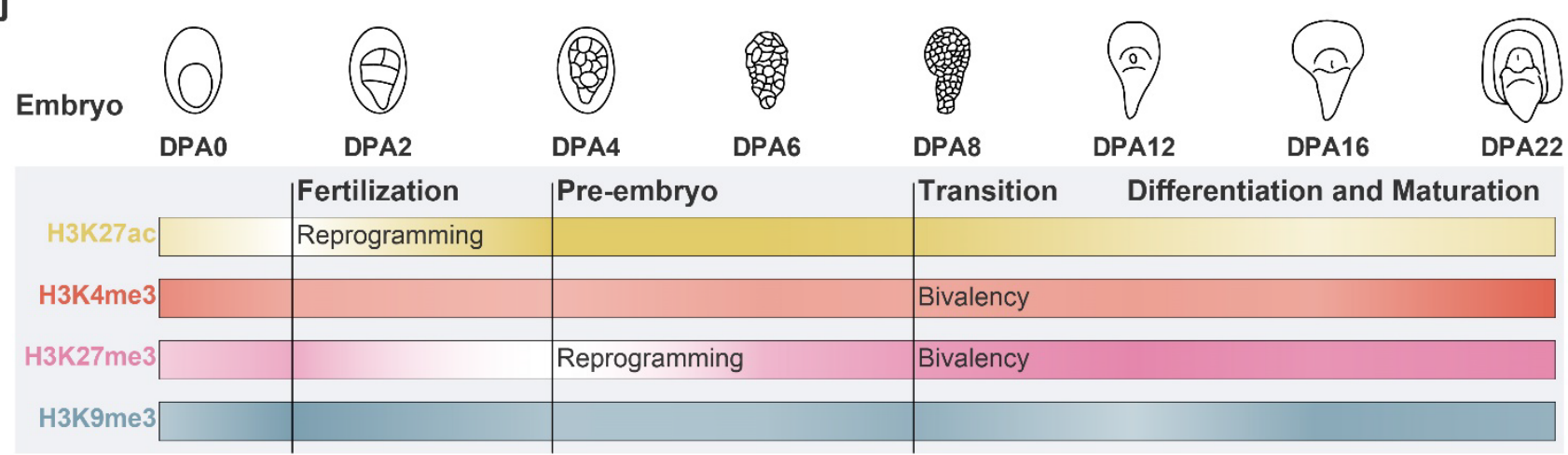




\section{Fig. 3 | Reprogramming of histone modifications during wheat embryogenesis}

a, Emission probabilities for histone modifications in 15 ChromHMM states, which could be mainly categorized in five groups: $\operatorname{Pr}$ (promoter), EnL (enhancer-like), $\operatorname{Tr}$ (transcription), PcG (polycomb group), and Hc (heterochromatin) state. The abbreviation following dash: A: active; W: weak; B: bivalency; P: poised; S: strong; I: initiation; E: elongation; H: H3K9me-associated; Ns: No signal. b, Chromatin states transition among different developmental stages.

c, Dynamic of five major chromatin states during different embryonic stages.

d, Percentage of changed chromatin state coverage to the total genome size among different embryonic stages. Colors filled represent the five major chromatin state groups as shown in $\mathbf{a}$. e, Histone modification dynamic in promoter and genic regions during wheat embryogenesis. f, Regions of H3K4me3, H3K27me3 and bivalency at different developmental stages. g, Scatter plots correlating H3K27me3 (x axis) and H3K4me3 (y axis) enrichments $\left(\log _{2} \mathrm{CPM}\right.$ ) at proximal region. Different colors in the left panel indicate various modification states as labeled and chromatin accessibility of correlated plots is shown in the right panel with color bar for relative intensity $\left(\log _{2} \mathrm{CPM}\right)$.

h, Fractions of all protein-coding and TF-coding genes marked by bivalent promoter at five major developmental stages.

i, Comparisons of histone modifications H3K27ac, H3K4me3, H3K27me3 and H3K9me3 of collinear regions between Chr. 5A and Chr. 5B. Down- (Dn), and Up-regulated (Up) loci were defined by the $\log _{2}$ (fold change $\mathrm{Chr}$. 5B/ Chr. 5A) values bigger and lower than 1 and -1 , respectively. $\mathrm{Nc}$ indicates no change. Color bars and grey lines indicate the chromosome segments and centromere regions as those in Fig. $2 \mathrm{~d}$.

j, Schematic represents the chromatin state dynamic during wheat embryogenesis. The dark and light colors represent the strength and weakness of the signal, respectively. 
bioRxiv preprint doi: https://doi org/10.1101/2022 0121.477188; this version posted January 23, 2022. The copyright holder for this

a
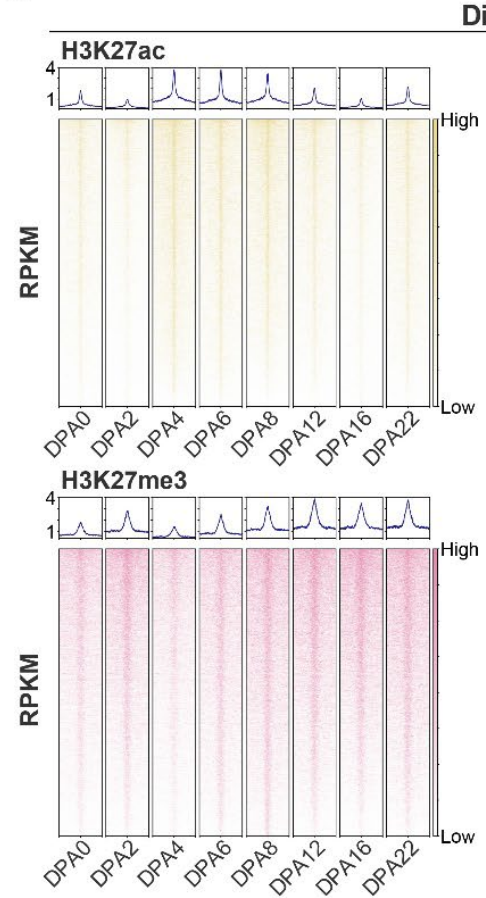

Distal
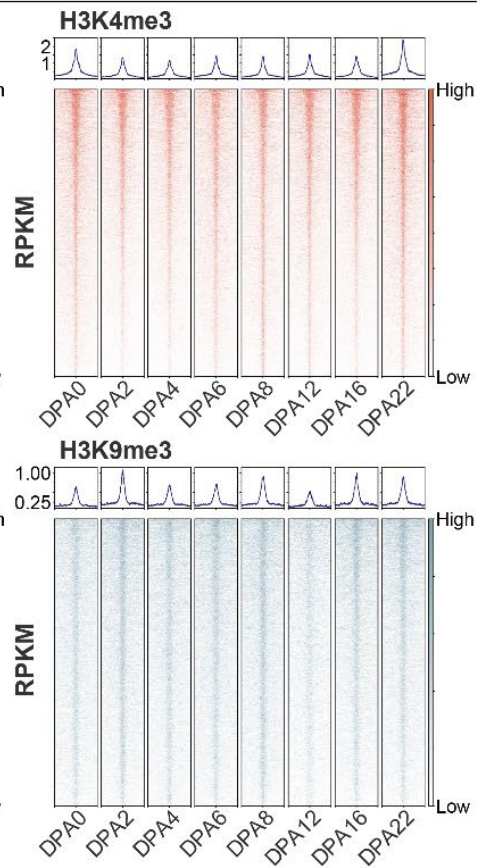

b
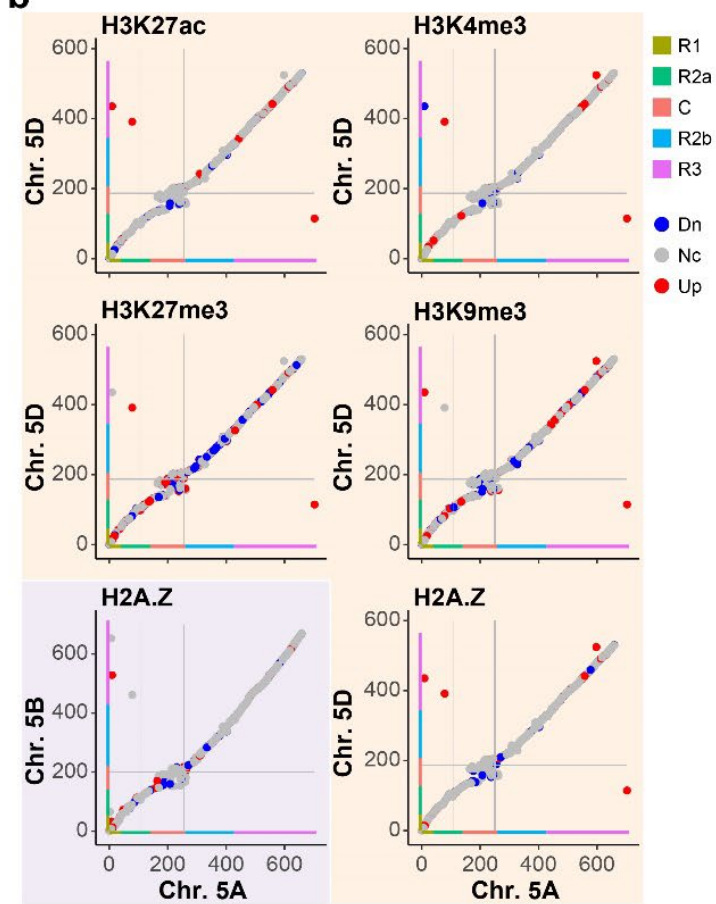

C

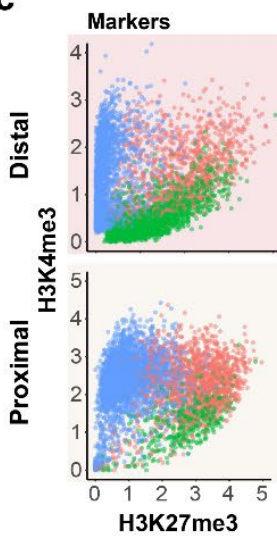

RNA-seq

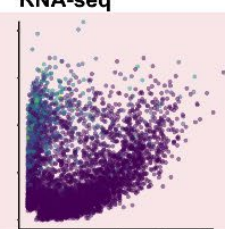

Define

Bivalency

- H3K27me3

- H3K4me3

Relative enrichment

High
Low
H3K27ac

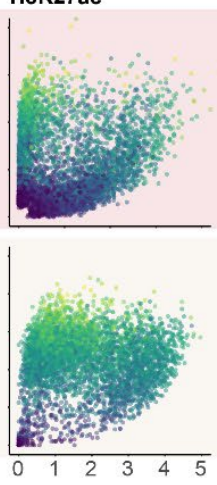

H3K9me3

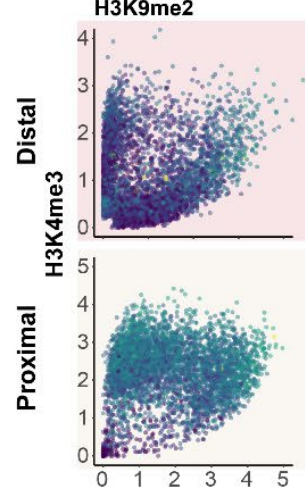

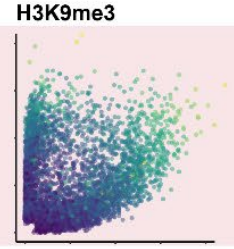

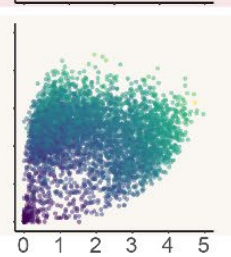

H3K9ac

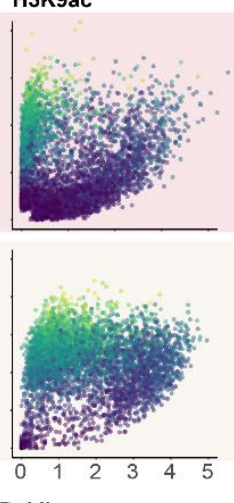

Pol II
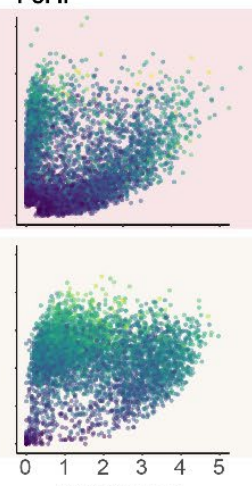

H3K27me3
H3K36me3

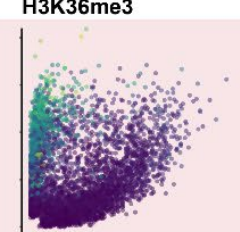

H3K4me1

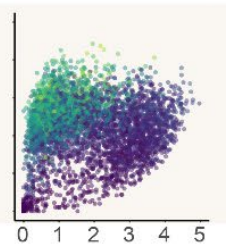

H2A.Z
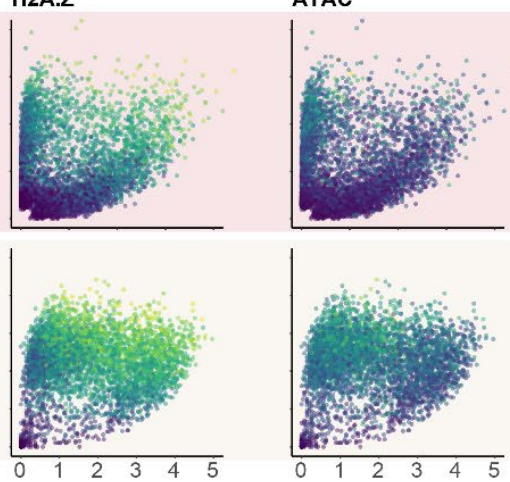
Fig. S5 | Histone modifications dynamic and subgenome comparison

a, Histone modifications dynamic in distal regions during wheat embryogenesis.

b, Comparisons of histone modifications H3K27ac, H3K4me3, H3K27me3, H3K9me3 and H2A.Z of collinear regions between three subgenomes of Chr. 5. Down- (Dn), and Up-regulated (Up) loci were defined by the $\log _{2}$ (fold change $\mathrm{Chr}$. 5B or Chr. 5D / Chr. 5A) values bigger and lower than 1 and -1 , respectively. Nc indicates no change. Color bars and grey lines indicate the chromosome segments and centromere regions as those in Fig. $2 \mathrm{~d}$.

c, Scatter plots correlating H3K27me3 (x axis) and H3K4me3 (y axis) enrichments ( $\log _{2} \mathrm{CPM}$ ) at distal and proximal regions. Color represents chromatin state defined as indications, gene expression and multiple histone modifications level $\left(\log _{2} \mathrm{CPM}\right)$. 
bioRxiv preprint doi: $\mathrm{https}$ //doi.org/10.1101/2022.01.21.477188; this version posted January 23, 2022. The copyright holder for this preprint (which was not certified by peer review) is the author/funder. All rights reserved. No reuse allowed without permission.

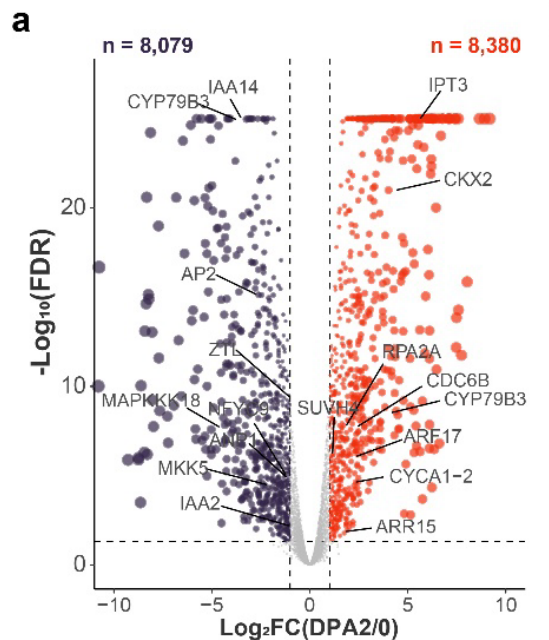

(2)
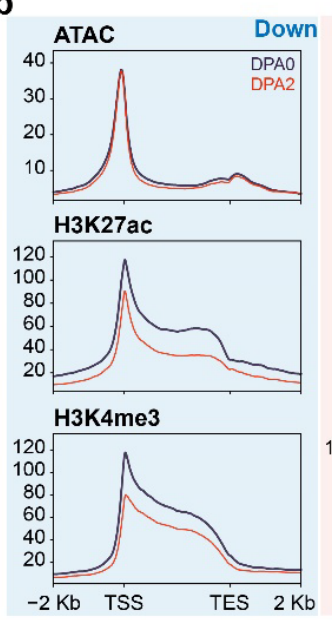

C

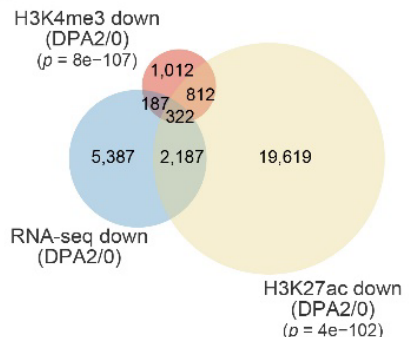

d

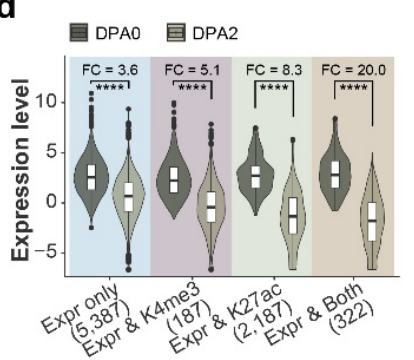

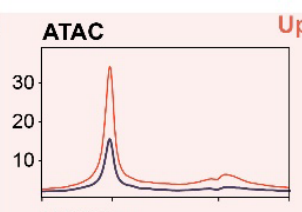

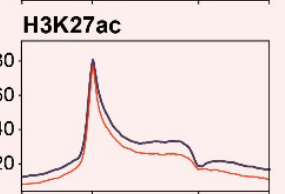

e
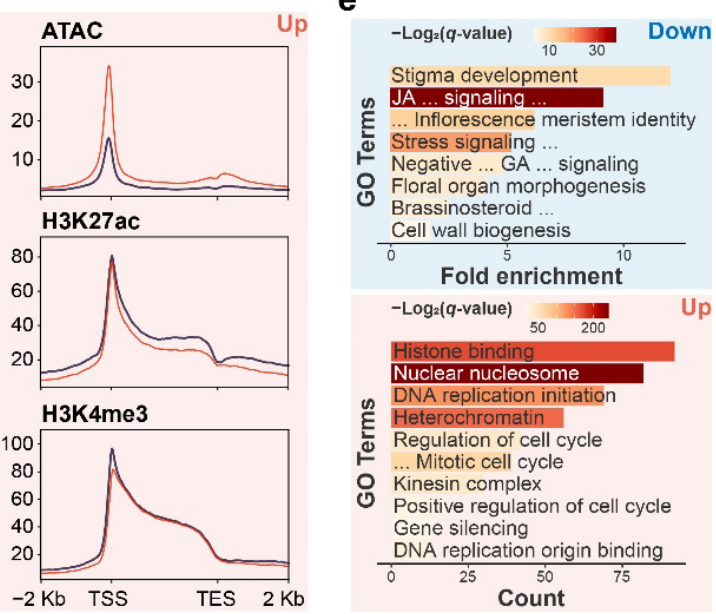

f

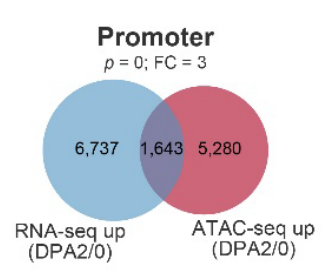

g

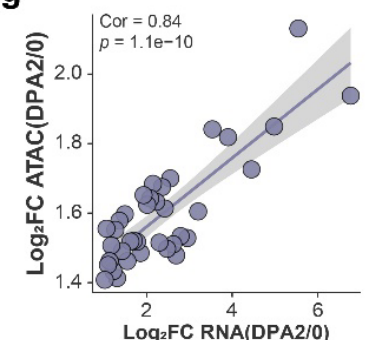

h

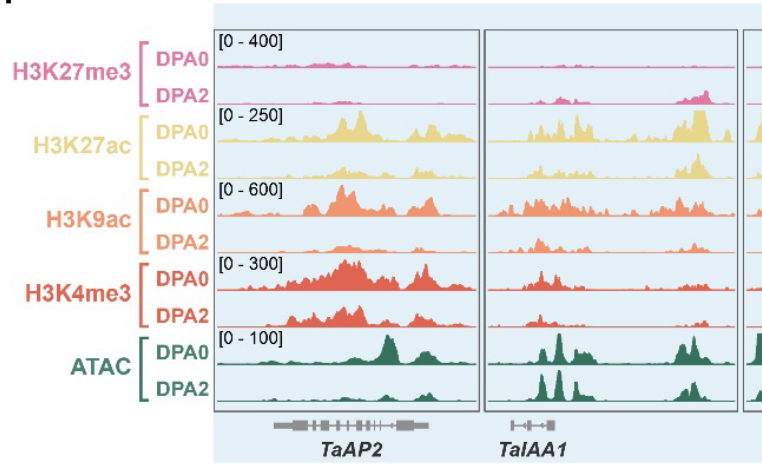

i

Proximal regulation Homologs of ZGA genes in Arabidopsis

$\longrightarrow$ Distal regulation Specific ZGA genes in Wheat

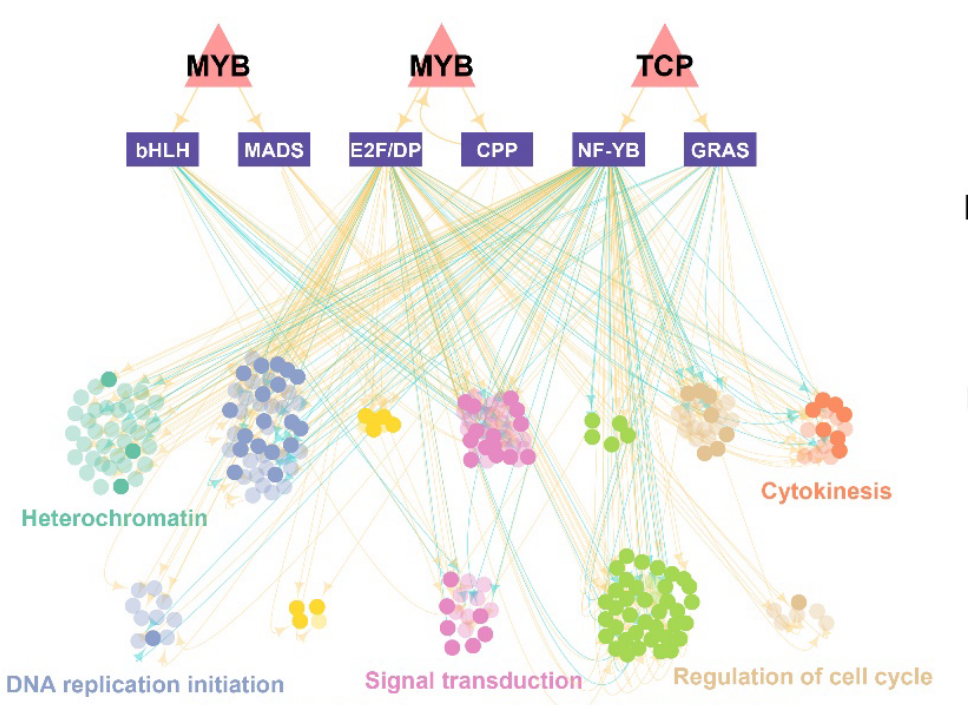

DNA replication initiation
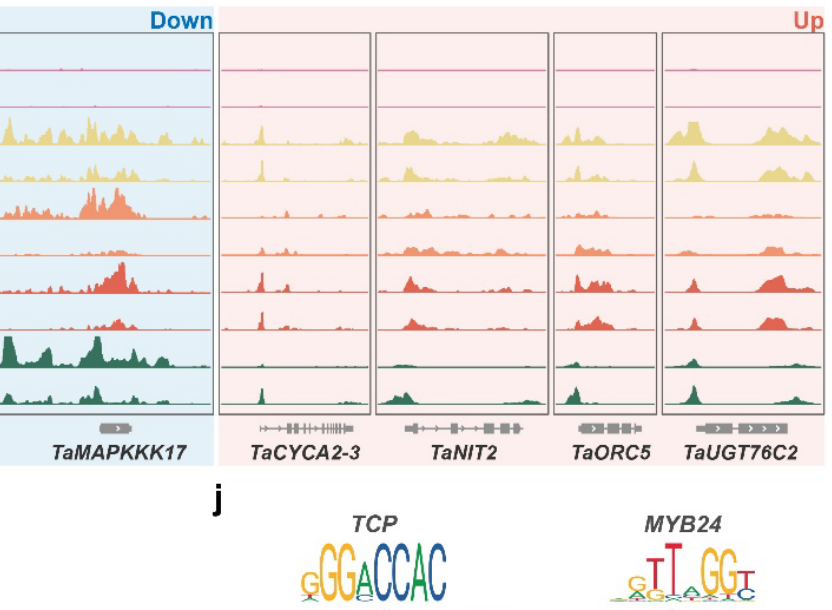


\section{Fig. 4 | Transcription and chromatin reconfiguration during MZT}

a, Volcano plot of differentially expressed genes during MZT. DEGs were defined by the threshold value of $\log _{2}$ (Fold Change DPA2/DAP0) $\geq 1$ and FDR $\leq 0.05$ by edgeR.

b, Chromatin accessibility and histone modification profiles of down- (left) and up-regulated (right) genes.

c, Overlap between down-regulated genes and corresponding active histone modification marks decreasing.

d, Expression levels of different gene sets generated from c.

e, GO enrichment for 2,696 down-regulated genes in which active histone modification marks decreased (c) (top) and 1,643 up-regulated genes in which gained pACRs (f) (bottom).

f, Overlap between up-regulated genes and corresponding chromatin accessibility gains.

g, Correlation between gained pACRs and up-regulated genes based on 1643 genes overlapped. Genes were ranked by RNA-seq fold change and separated into 50 bins.

h, IGV showing histone modifications and chromatin accessibility changes on representative downregulated genes $A P 2, M A P K K K 17$, as well as $I A A 1$ (light blue shade), and up-regulated genes CYCA2-3, NIT2, ORC5, as well as UGT76C2 (light red shade).

i, GRNs for hierarchical regulation of zygotic active genes sets. Both proximal (orange) and distal (green) regulations were included. The Upper and lower layers of GRN represented the predicted regulation hierarchy. Each point represented a TF or gene. The target genes with orthologs of Arabidopsis ZGA genes were represented in light color, while the specific genes were represented in dark color.

j, ATAC-seq footprint of key TFs in i during MZT.

$\mathbf{k}$, Expression patterns of TCP by in situ at DPA 0, 2 and 4.

1, Luciferase reporter assays validation of transcriptional activation capability of TCP on targets TaNF-YB and TaGRAS.

Hypergeometric distribution test was used for $\mathbf{c}$ and $\mathbf{f}$. Mann-Whitney $\mathrm{U}$ test (two-sided) and

Student's t test were used for $\mathbf{d}$ and $\mathbf{l}$, respectively $\left(*: \mathrm{p}<=0.05 ; * *: \mathrm{p}<=0.01 ;{ }^{* * *}: \mathrm{p}<=0.001\right.$; $* * * *: \mathrm{p}<=0.0001)$. Pearson correlation test was used in $\mathbf{g}$. 
a

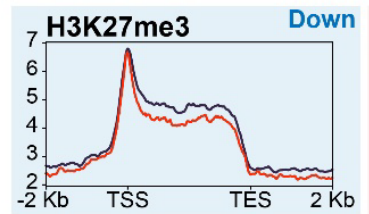

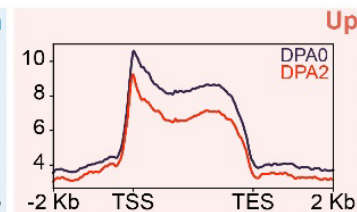

C
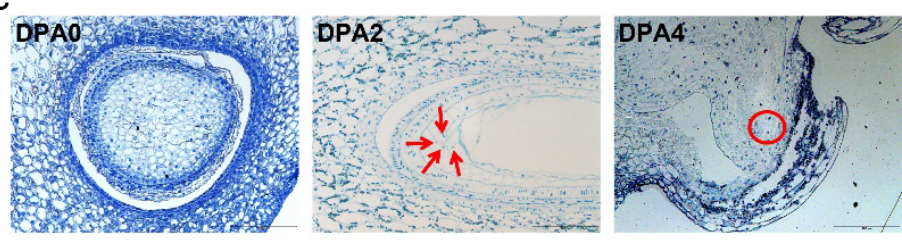

b

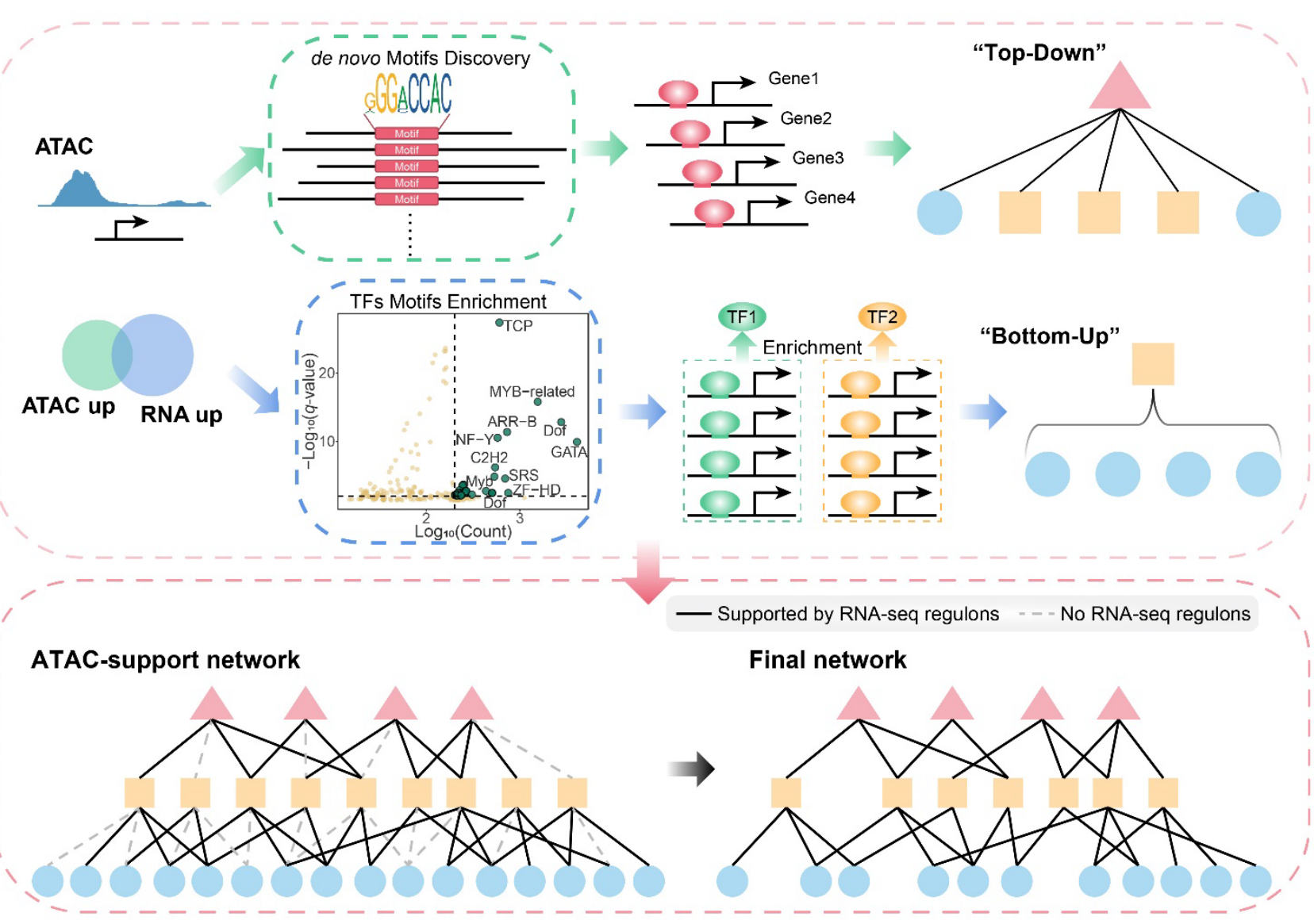

Fig. S6 | Histone modification change and build-up of transcription regulation network for ZGA

a, H3K27me3 change following fertilization at dn- and up-regulation gene sets as Fig. $4 \mathrm{~b}$.

b, Schematic of the strategy for GRNs building (see method for detail).

c, Morphological change of embryo-sac during early embryogenesis. 
bioRxiv preprint doi: https://doi org/10.1101/2022.01.21.477188; this version posted January 23, 2022. The copyright holder for this preprint (which was not certified by peer review) is the author/funder. All rights reserved. No reuse allowed without permission.

a

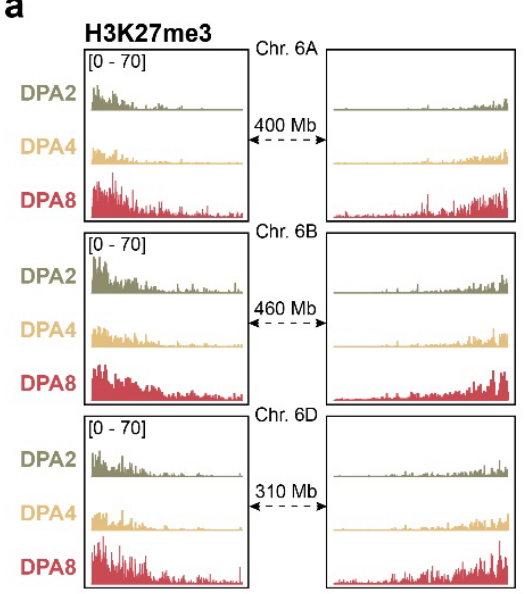

C

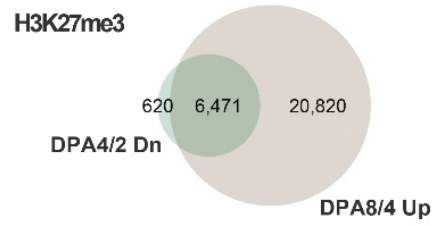

d

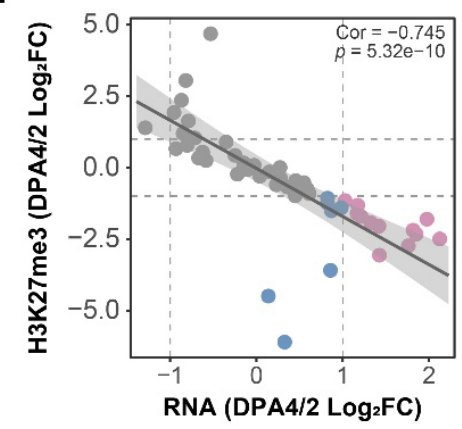

g
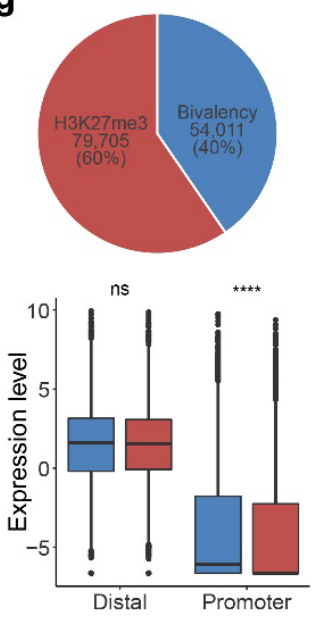

b

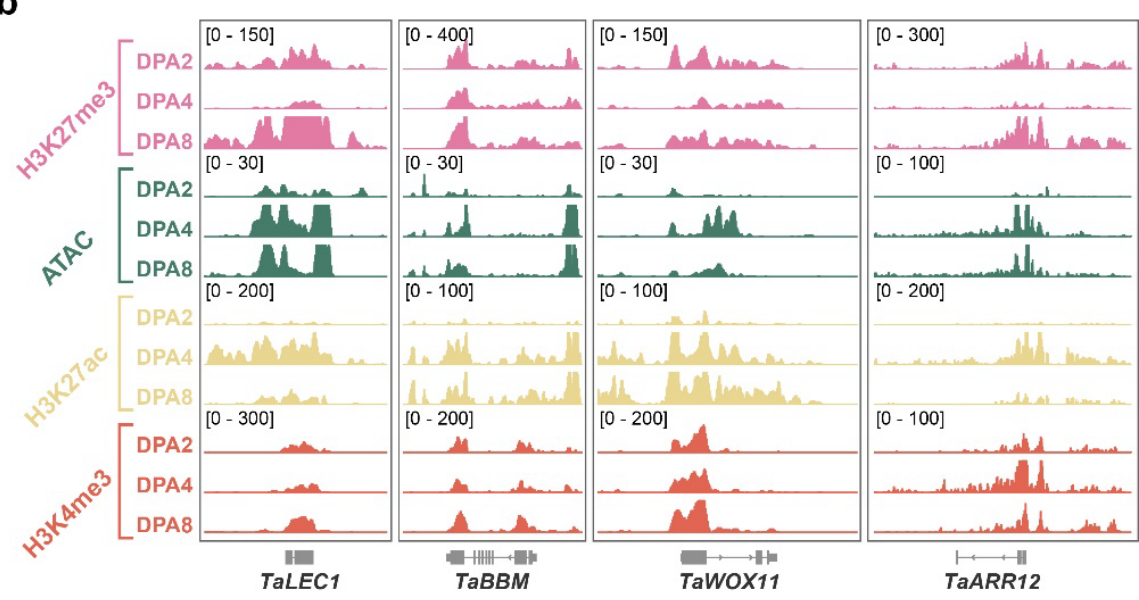

e
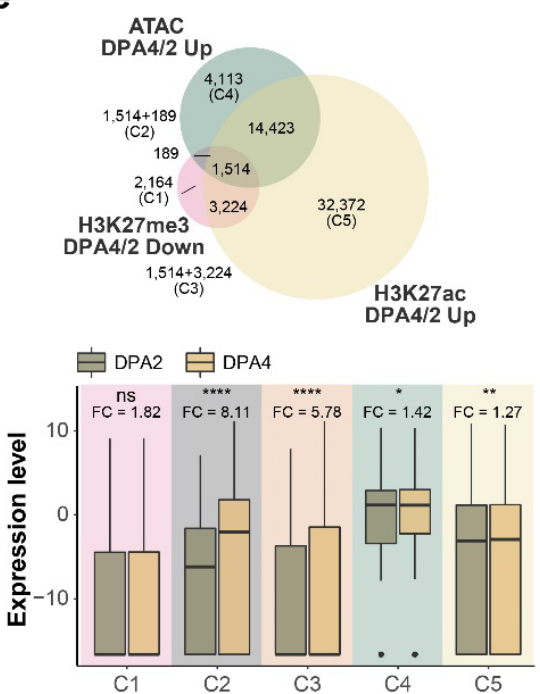

f

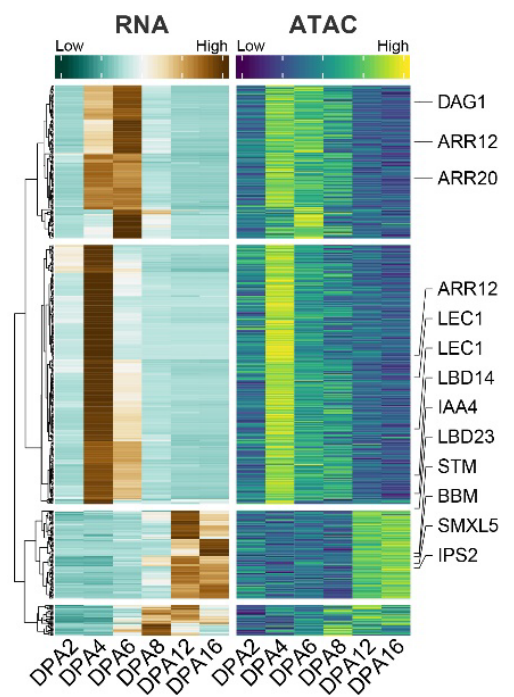

i

H3K27me3H3K4me3 ATAC
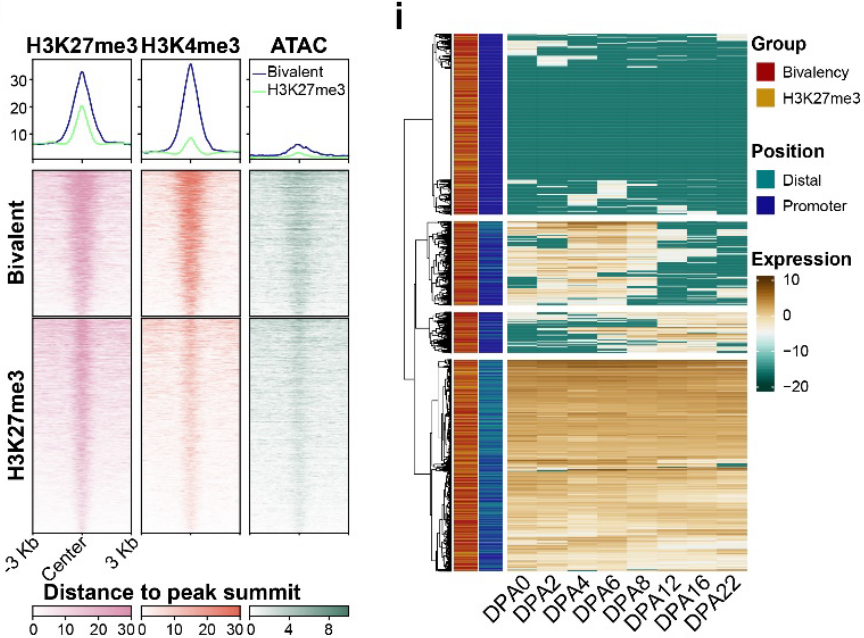

j

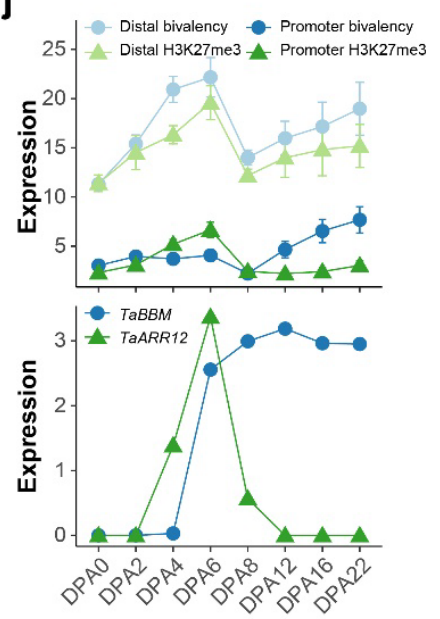




\section{Fig. 5 | H3K27me3 reprogramming ensures pre-embryo development}

a, Dynamic of H3K27me3 modification in Chr. 6 during embryogenesis, with resetting at DPA4 and regaining at DAP8.

b, IGV showing histone modifications and chromatin accessibility changes on embryo developmental essential genes.

c, Overlap between genes with H3K27me3 decreasing at DPA4 and increasing at DPA8.

d, Correlation between H3K27me3 decreasing and target genes' activation from DAP4 to DPA2. Genes were ranked by RNA-seq fold change and separated into 50 bins. H3K27me3 loss with mRNA level increased genes are shown in red color, while loss of H3K27me3 without mRNA changed is shown in blue color, while gray color indicates no change of H3K27me3.

e, Overlap among genes with down-regulated H3K27me3 and up-regulated ATAC-seq and H3K27ac modification (top), and altered expression levels of different gene sets (bottom).

f, Synchronous pattern between the gain of chromatin accessibility and elevation of gene expression on the basis of H3K27me3 decreasing at DPA4. Several developmental essential genes were highlighted.

g, Proportion of H3K27me3 lonely and bivalent regions following H3K27me3 increasing at DPA8 (top), and the expression level of corresponding target genes. Distal targets assignment was based on reg-dACR-target links from Fig. 2 h.

h, Histone modifications and ATAC-seq enrichment at H3K27me3 lonely or bivalent regions.

i.j, Expression pattern of genes associated with H3K27me3 lonely or bivalent modification across wheat embryogenesis. Heatmap showing clustering of gene expression (i), Line chart indicates the mean expression of various types of genes as indicated (top), as well as representative genes TaBBM (promoter bivalency) and TaARR12 (promoter H3K27me3 alone) were shown (bottom) (j). 
a

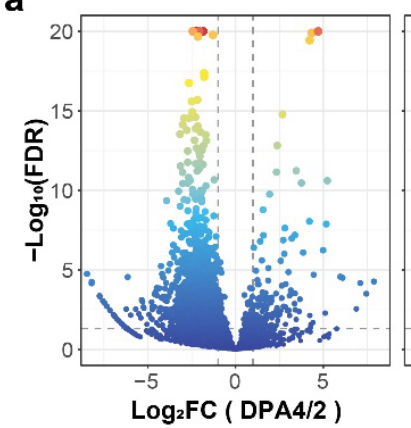

d

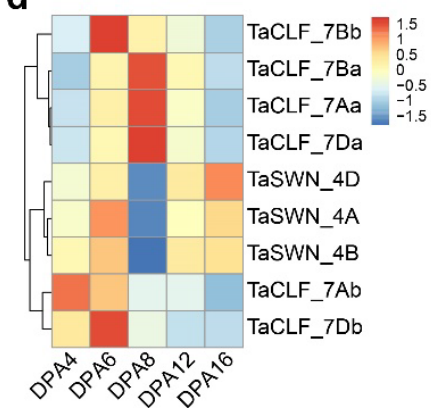

e

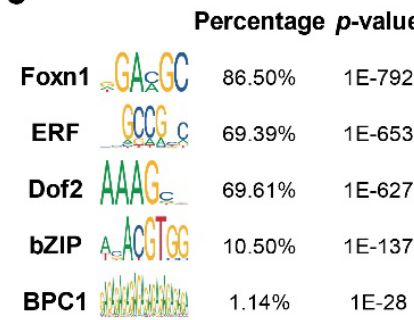

b

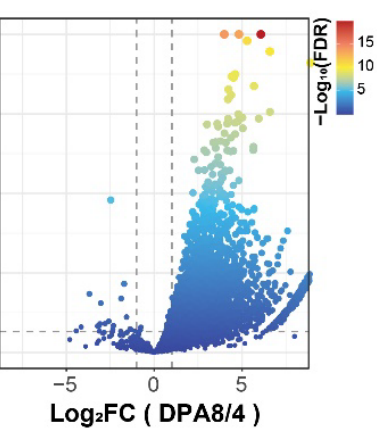

f

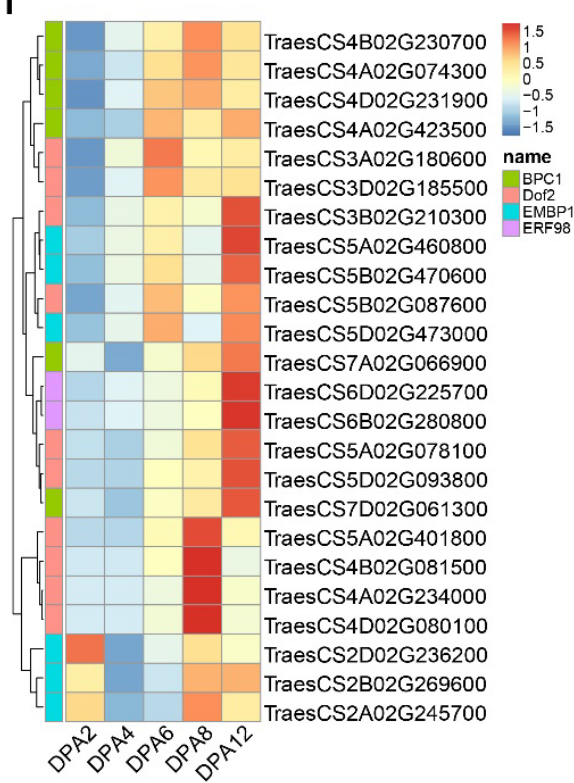

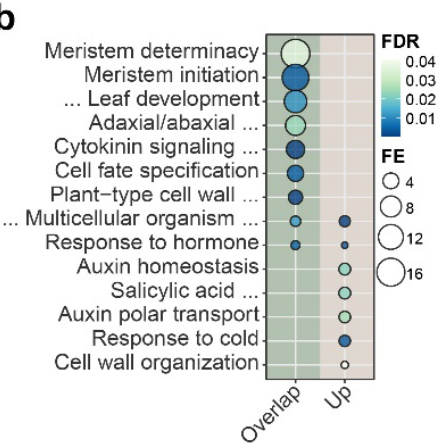

C

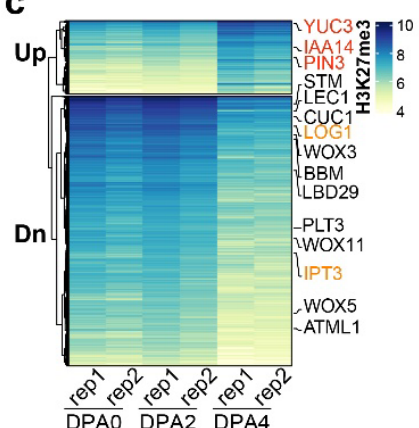

g

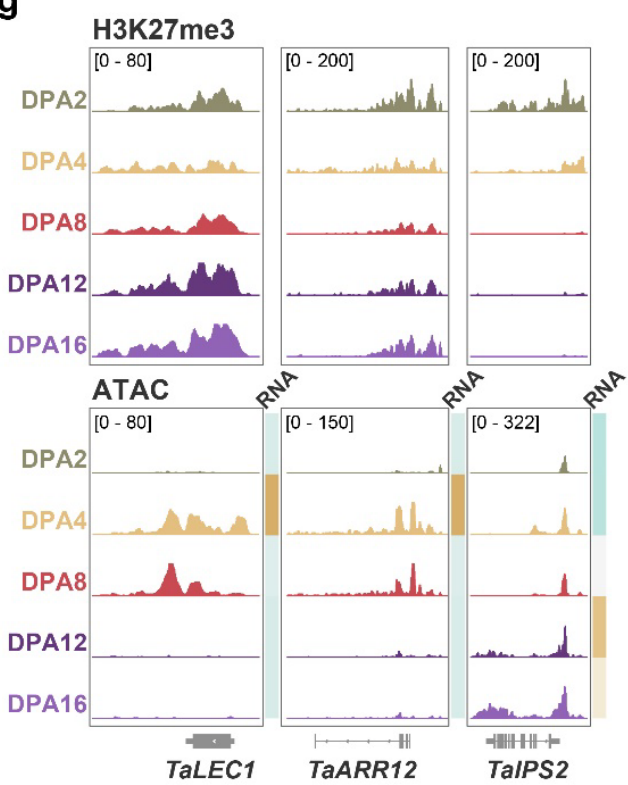

Fig. S7 | H3K27me3 reprogramming at pre-embryo

a, Volcano plot of differential H3K27me3 enriched regions between DPA2 and DPA4 (left), and between DPA4 and DPA8 (right). Differential regions were defined by the threshold absolute value of $\log _{2}$ (Fold Change) $\geq 1$ and FDR $\leq 0.05$ by DESeq 2 .

b, GO enrichment of gene sets which lost H3K27me3 at DPA4 and re-gain at DPA8, and gene sets which de novo gain of $\mathrm{H} 3 \mathrm{~K} 27 \mathrm{me} 3$ at DPA8.

c, Heatmap showing differential H3K27me3 modification genes between DAP4 and DAP2. Embryonic development essential genes were highlighted. Auxin-related genes were labeled in red and CK-related genes were labeled in orange.

d, The expression level of H3K27me3 methyltransferase coding genes in selected embryonic development stages.

e, f, Motif enrichment of H3K27me3 deposition loci at DAP8 (e) and the expression levels of cognate binding TFs (f). Homer was used for motif enrichment analysis.

g, H3K27me3 and chromatin accessibility dynamics at embryo essential genes TaLEC1, TaARR12 and TaIPS2. 
bioRxiv preprint doi: https://doi org/10.1101/2022.01.21.477188; this version posted January 23, 2022. The copyright holder for this preprint (which was not certified by peer review) is the author/funder. All rights reserved. No reuse allowed without permission.

a

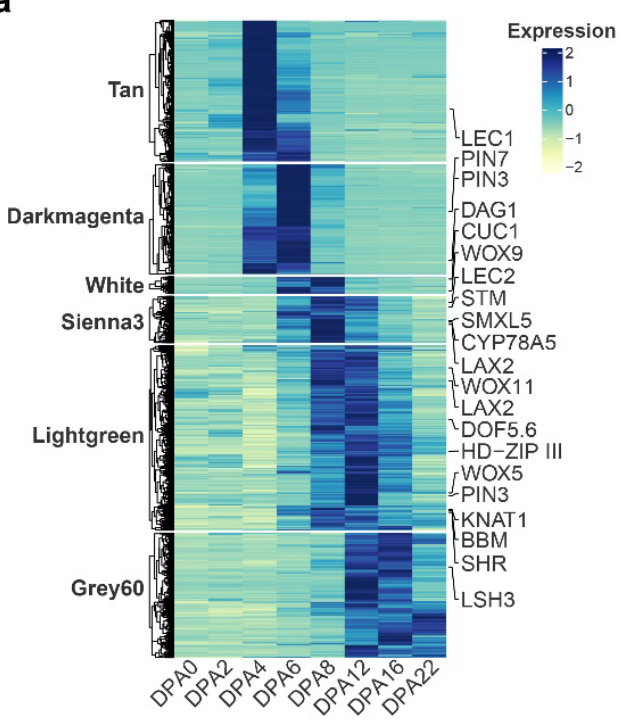

C

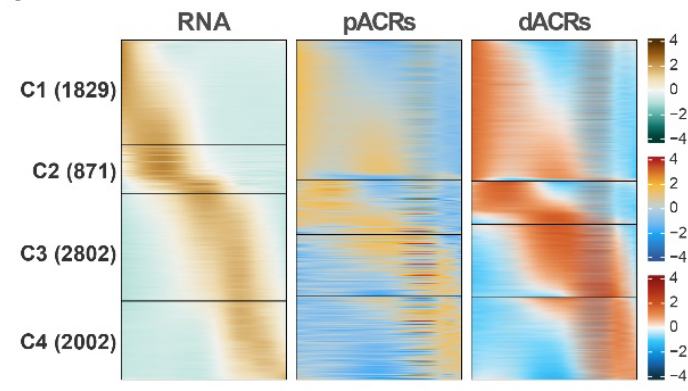

g

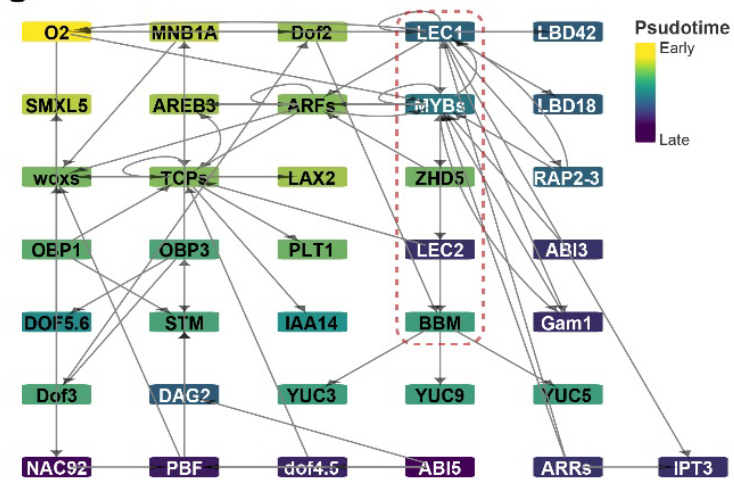

b
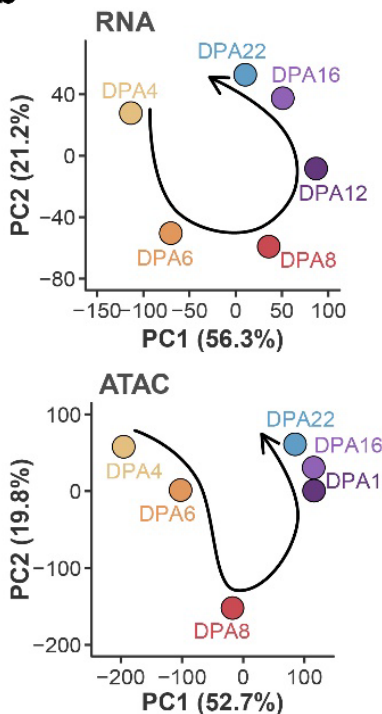

d

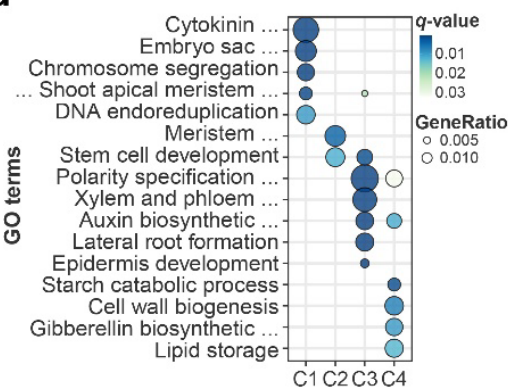

e

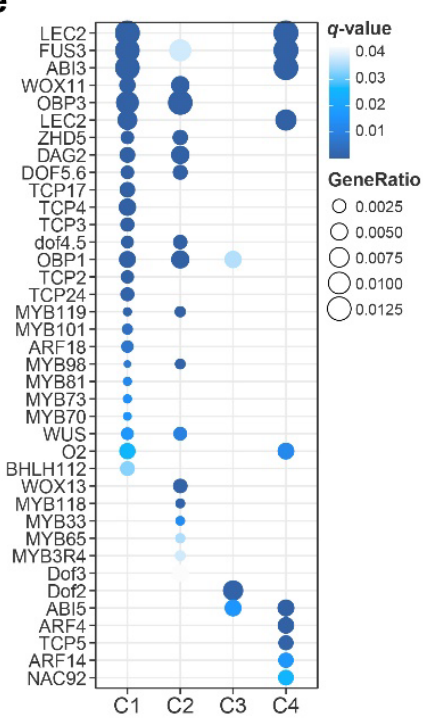

f

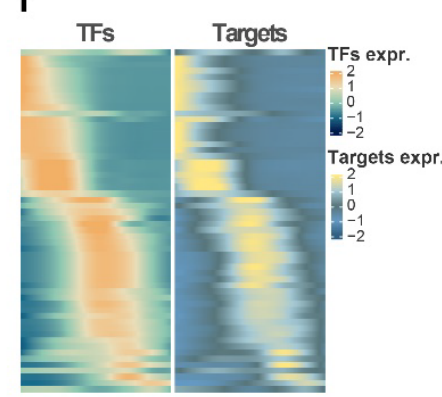

h

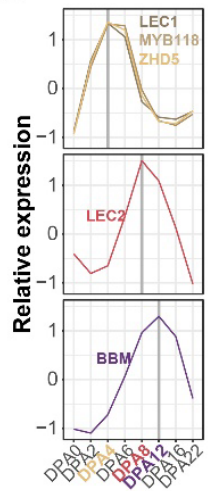

i

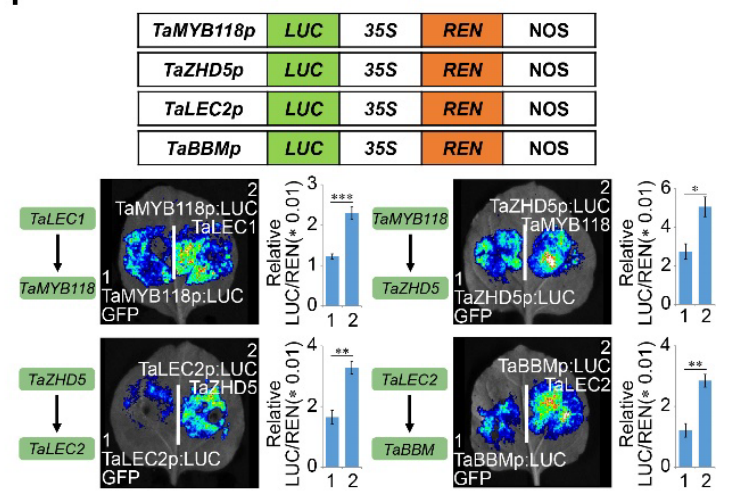




\section{Fig. 6 | Transcription regulatory network governing embryonic patterning}

a, Expression heatmap of selected key modules genes from Fig.1c. Genes with ortholog identified to function during embryogenesis in other species were indicated.

b, PCA trajectories of genes expression (top) from (a) and corresponding chromatin accessibility (bottom). Developmental time units (DTU) values on the right panel were calculated based on the straight distance between two adjacent points and then scaled to 0 to 10 .

c, Sorted standardized temporal genes, pACRs, dACRs and expression profiles. Four clusters were generated based on expression order.

d,e, GO and TFs enrichment for four clusters of genes from (c).

f, Heatmap showing the synchronous patterns between TFs and target genes. Each row in heatmap represents links between one TF and corresponding genes.

g, GRNs for key TFs participated in embryonic patterning. Genes were roughly ranked by the expression order from top to bottom and from left to right. Colors indicate the expression timing wave during embryo patterning.

h, Expression pattern of representative key genes in the GRN network.

i, Luciferase reporter assays validation of transcriptional regulation among representative TF-target pairs. Student's t test was used for the statistic significance $(*: \mathrm{p}<=0.05 ; * * \mathrm{p}<=0.01 ; * * * \mathrm{p}<=$ $0.001)$. 
bioRxiv preprint doi: https://doi org/10.1101/2022.01.21.477188; this version posted January 23, 2022. The copyright holder for this preprint (which was not certified by peer review) is the author/funder. All rights reserved. No reuse allowed without permission.

a

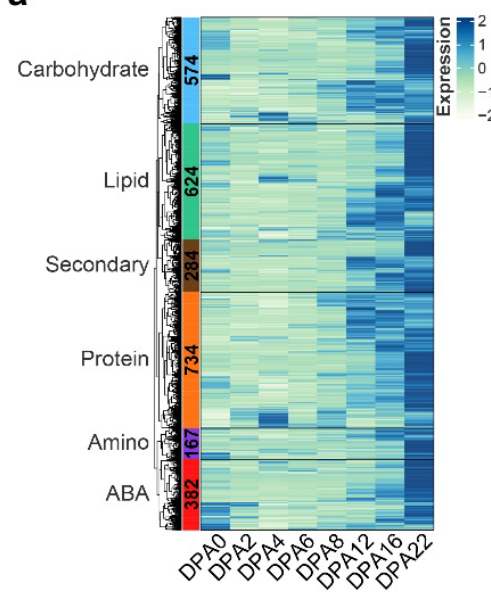

e

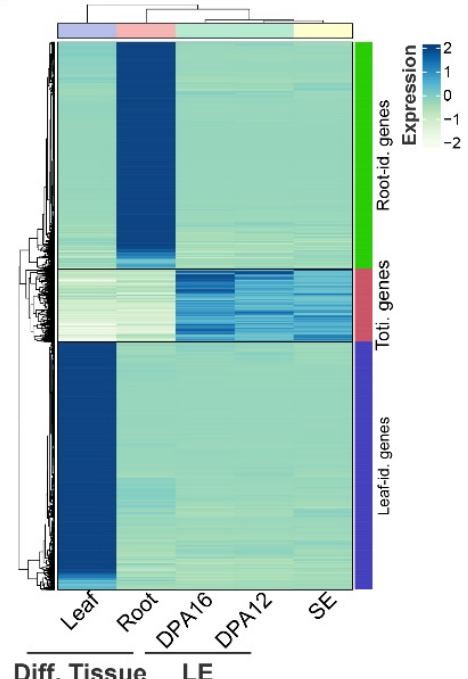

g

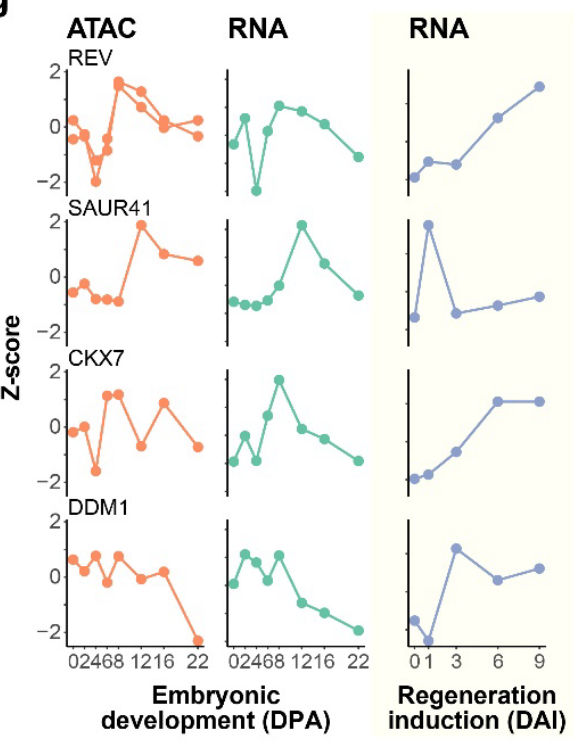

b
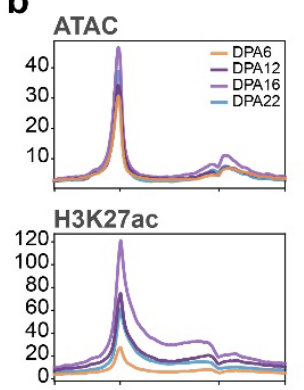

H3K27me3

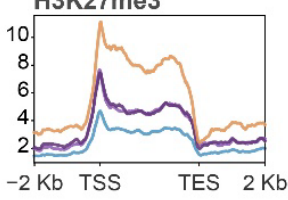

f

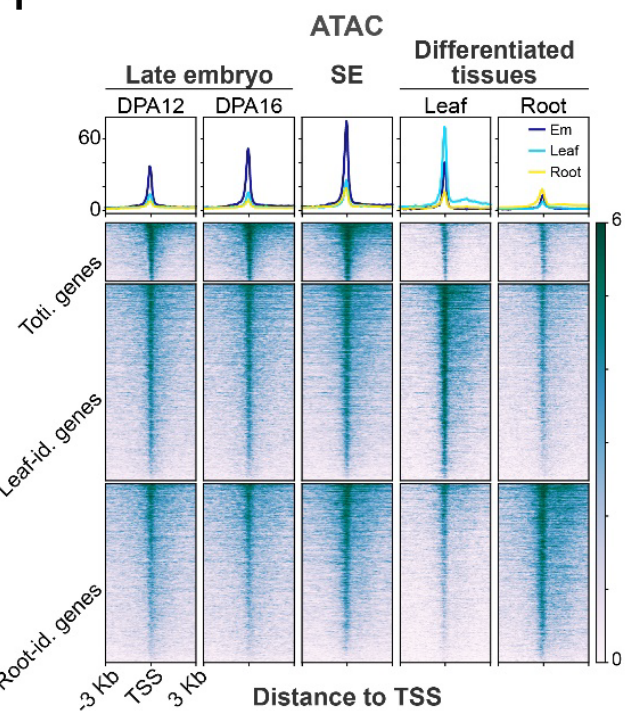

h
C

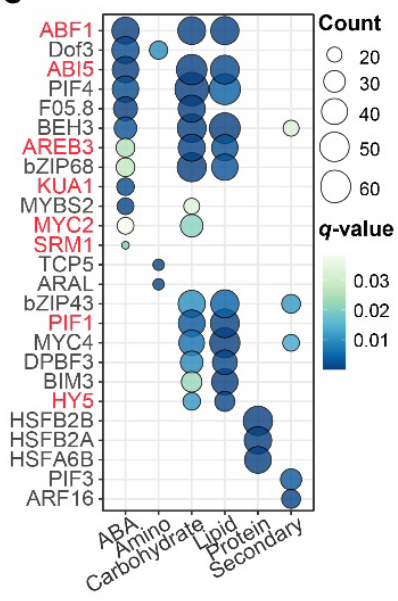

d

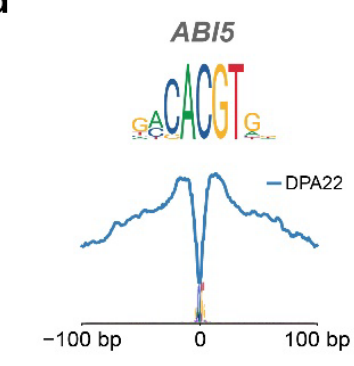

DPA22 ATAC footprint
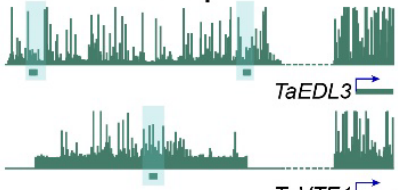

TaVTE1 $\longrightarrow$

H3K27me3

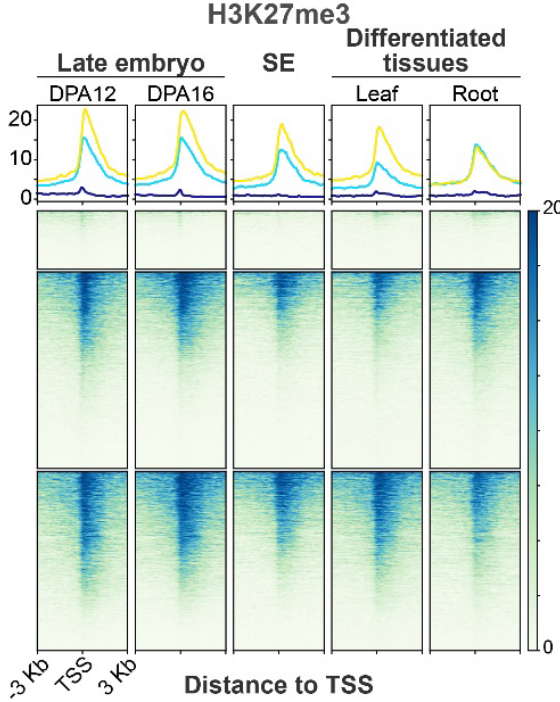

i \begin{tabular}{|l|l|l|l|l|}
\hline TaAMT1;1p & LUC & $35 S$ & REN & NOS \\
\hline
\end{tabular}
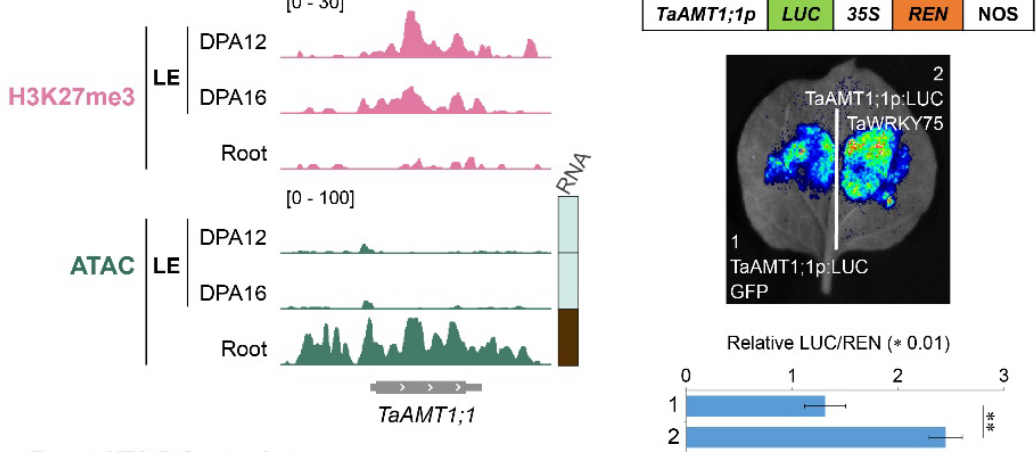

Root ATAC footprint

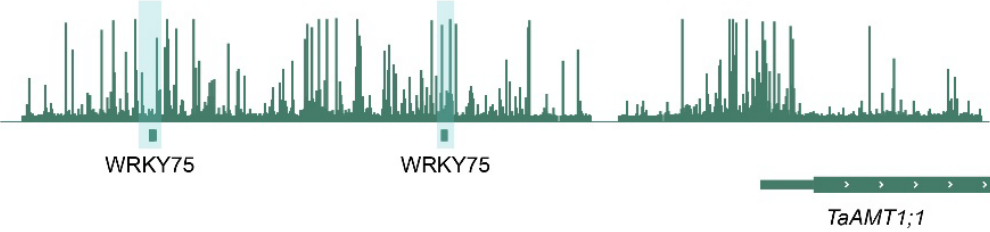




\section{Fig. 7 | Maturation of embryogenesis in the context of chromatin regulation}

a, b, Six pathway genes highly expressed during the late embryonic development (a), and corresponding epigenetic modifications (b).

c, d, Regulation of six pathway genes by specific cis motif and trans-factors. Binding TF enriched for each pathway genes (c). ABI5 footprint was highlighted (d top), and corresponding binding site at the promoters of carbohydrate-related gene TaEDL3 and ABA-related gene TaVTE1 (d bottom).

e, Identification of organ identity and totipotent genes based on expression specification across different tissues.

f, ATAC and $\mathrm{H} 3 \mathrm{~K} 27 \mathrm{me} 3$ modification on the promoters of different genes sets in e cross five tissues, SE: somatic embryo.

g, Chromatin accessibility (left) and expression (mid) of regeneration genes during wheat embryogenesis, and their expression during callus induction (right).

h, H3K27me3 and chromatin accessibility regulated root-identity gene TaAMT1;1 specific expression pattern in root and late embryo (LE). The ATAC footprint tracks showing the binding sites of TaWRKY75, a key TF predicted to bind TaAMT1;1 as indicated in supplementary Fig. 8e. i, Luciferase reporter assays show the transcriptional activation capability of TaWRKY75 to TaAMT1; 1 in $N$. benthamiana leaves. Student's $t$ test was used for the statistic significance $(*: \mathrm{p}<=$ $0.05 ; * *: \mathrm{p}<=0.01 ; * * *: \mathrm{p}<=0.001)$. 
a

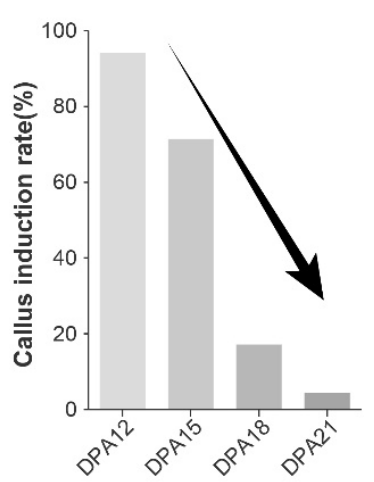

d

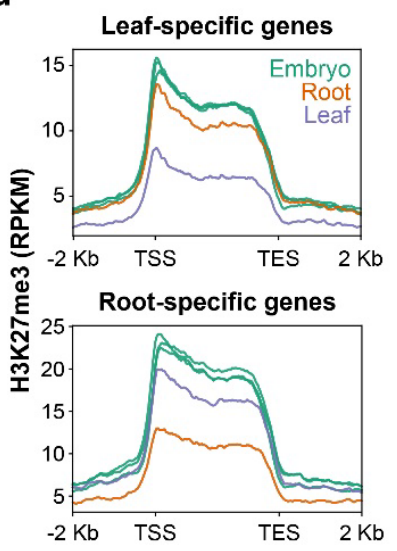

b

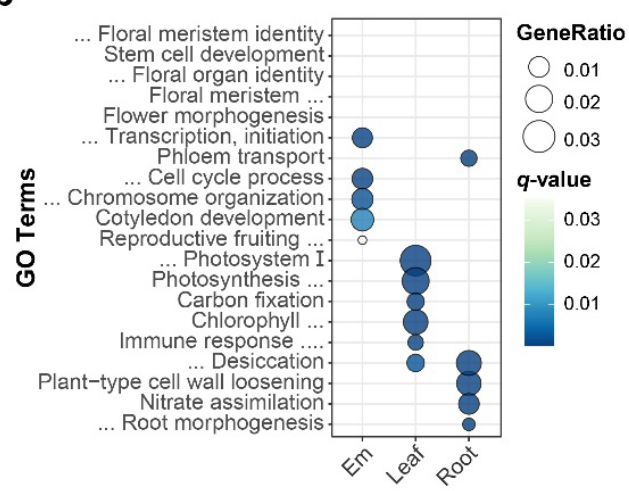

C

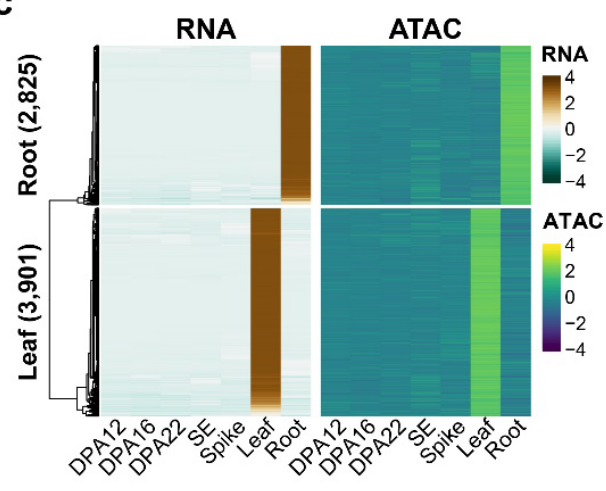

e

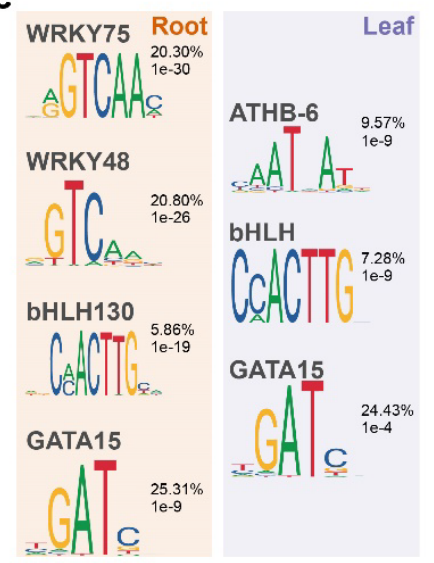

f

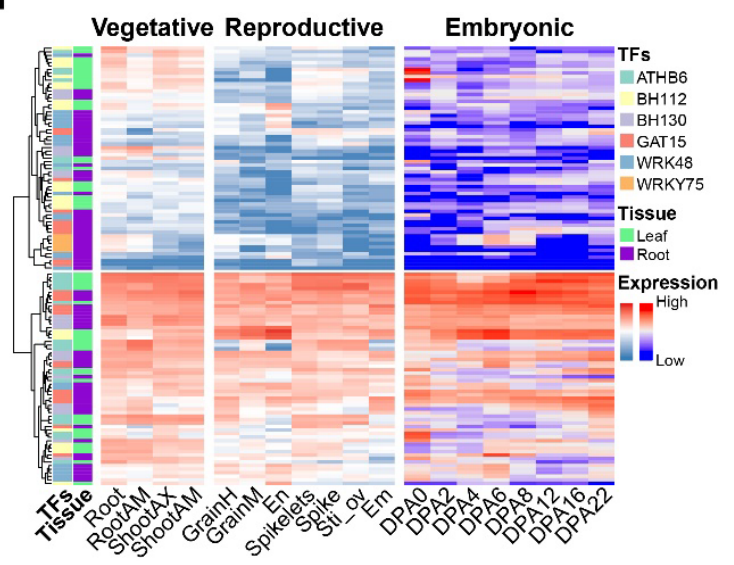




\section{Fig. S8 | Inhibition of deep organogenesis in late embryo at chromatin level}

a, Callus induction rate drops as embryo developing after DPA 12.

b, GO enrichment of tissue identity genes found in Fig. 7d.

c, Synchronous patterns between root- and leaf-identity genes expression and corresponding pACRs in individual tissue.

d, H3K27me3 modification levels on root- and leaf-specific genes in individual tissue.

e, Motifs enrichment within regulatory regions of root- (left) and leaf-specific genes (right).

Percentage showed the presence frequency, while P-value indicates the enrichment.

f, Expression patterns of candidate TFs that can bind motifs found in e, cross different tissues and during embryogenesis. 
bioRxiv preprint doi: https://doi.org/10.1101/2022.01.21.477188; this version posted January 23, 2022. The copyright holder for this preprint (which was not certified by peer review) is the author/funder. All rights reserved. No reuse allowed without permission.

a
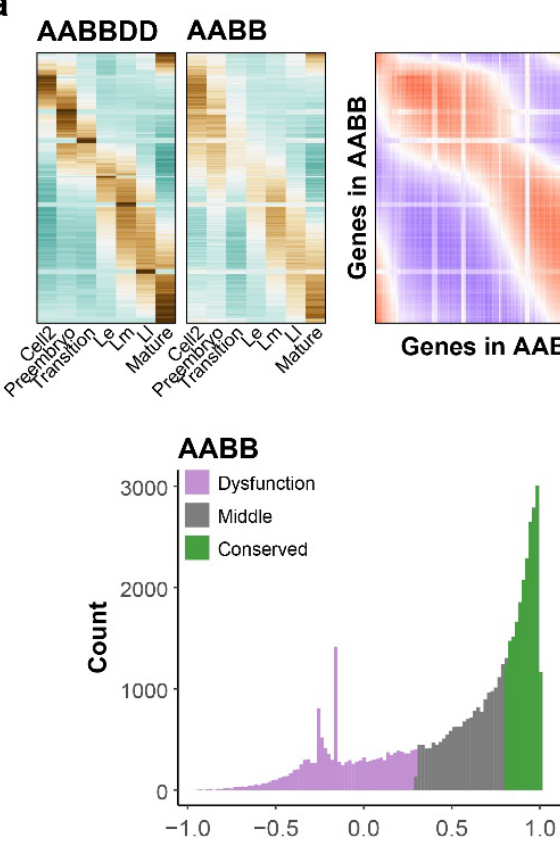

b

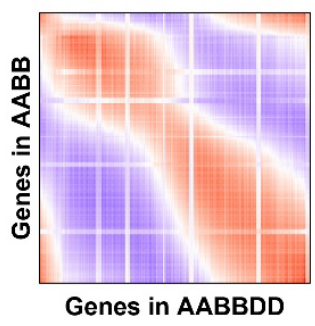

d

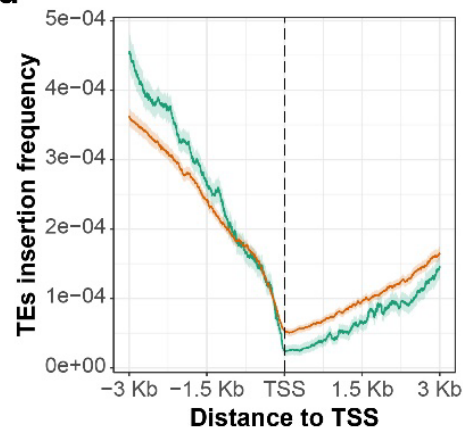

C
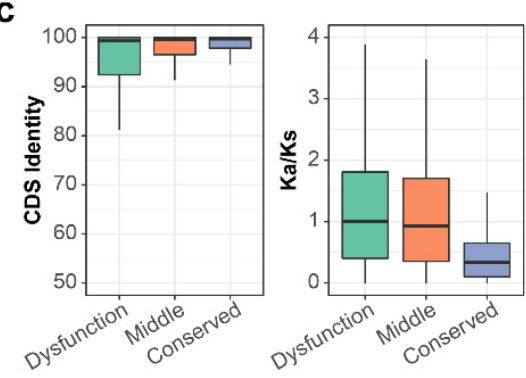

e

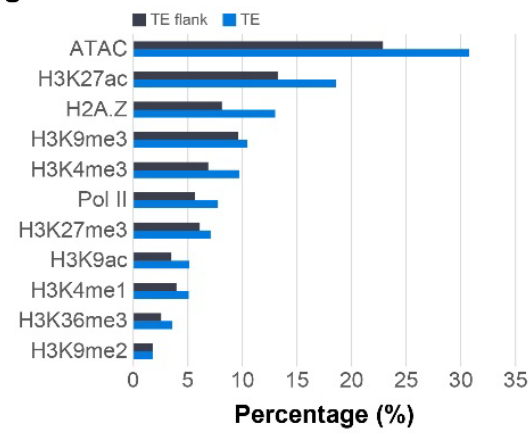

f
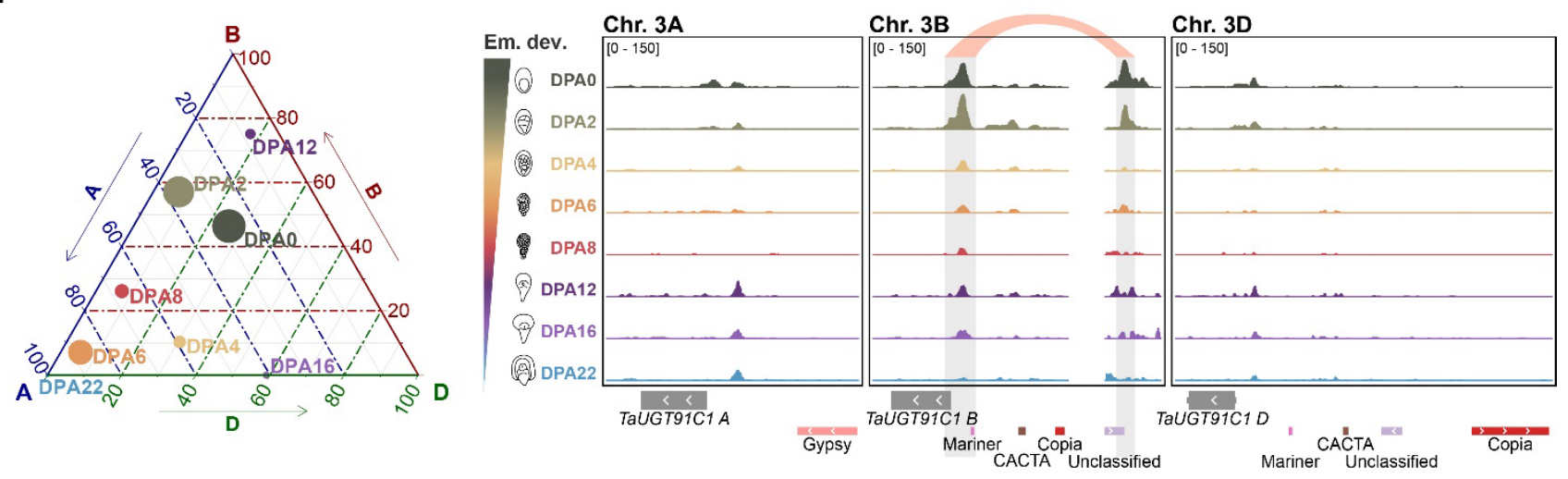

g
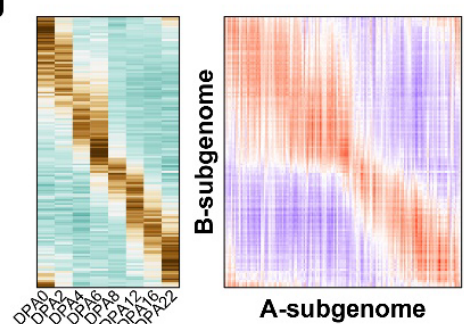

A-subgenome

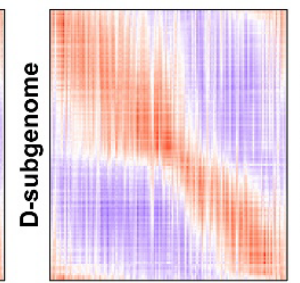

A-subgenome

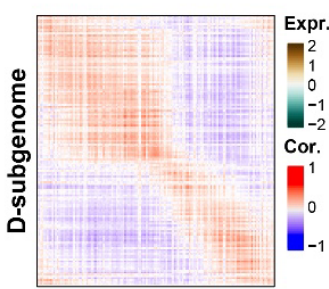

B-subgenome

h
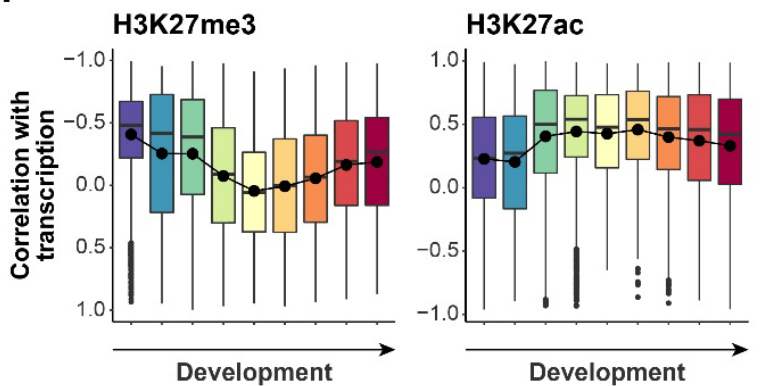

i

Inverse hourglass model

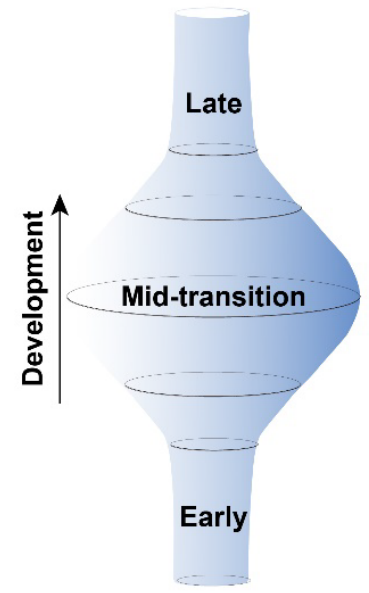

H3K4me3

H3K27me3

H3K27ac

TFs-binding

H3K4me3

H3K27me3

Transcription-distance 
Fig. 8 | Transcriptional divergence between different ploidy wheat and epigenetic governed inverse hourglass model

a, Comparisons of gene expressions between hexaploid wheat (AABBDD) and ancestor (AABB). Each row represents an individual gene in hexaploid wheat and its counterpart ancestor (top left). Pearson correlation index was calculated between genes expression in hexaploid wheat and counterpart in ancestor (top right). Genes were clustered into three categories based on Pearson index (bottom).

b, Genomic features differences between dysfunction and conserved cluster genes generated in a. Statistics were generated from supplementary Fig. 9b-f.

c, Coding sequence similarity and $\mathrm{Ka} / \mathrm{Ks}$ comparisons among different gene sets in $\mathbf{a}$.

d, TEs insertion frequency in the promoters of different gene sets in $\mathbf{a}$.

e, epigenetic modification enrichment at TEs insertion loci.

f, Homoeologs bias expression of representative gene TaUGT91C1 (left) and epigenetic regulations (right).

g, Comparisons of homoeologs expressions among subgenomes of hexaploid wheat. Each row represents an individual gene A-subgenome of hexaploid wheat, and the genes were ranked by activated order (left). Pearson correlation index was calculated between homoeologs expression in different subgenomes of hexaploid wheat as those in a.

h, H3K27me3 and H3K27ac contribution to genes expression across wheat embryogenesis (left) and key TFs binding strength at different developmental stages (right). The top 100 variable motifs calculated by chrVAR were used as key TFs.

i, The 'inverse hourglass model'. The transcription distance was large in the mid-stage. Histone modification $\mathrm{H} 3 \mathrm{~K} 4 \mathrm{me} 3$ and $\mathrm{H} 3 \mathrm{~K} 27 \mathrm{me} 3$ contributed to the early- and late-stage, and $\mathrm{H} 3 \mathrm{~K} 27 \mathrm{ac}$ and TFs-binding contributed to the mid-stage. 
bioRxiv preprint doi: https://doi org/10.1101/2022.01.21.477188; this version posted January 23, 2022. The copyright holder for this preprint (which was not certified by peer review) is the author/funder. All rights reserved. No reuse allowed without permission.

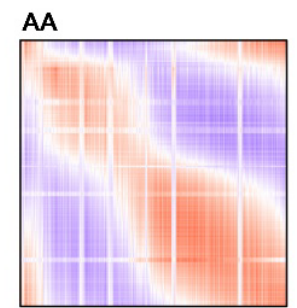

BB

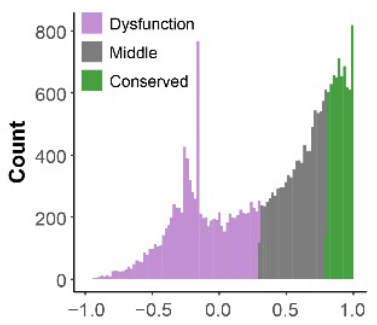

b

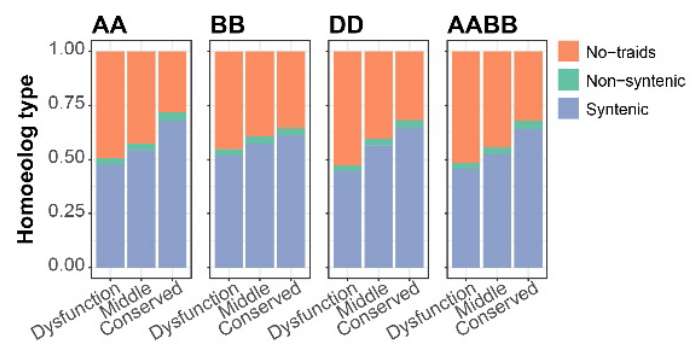

d

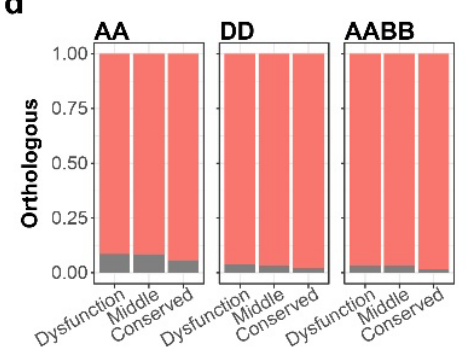

e
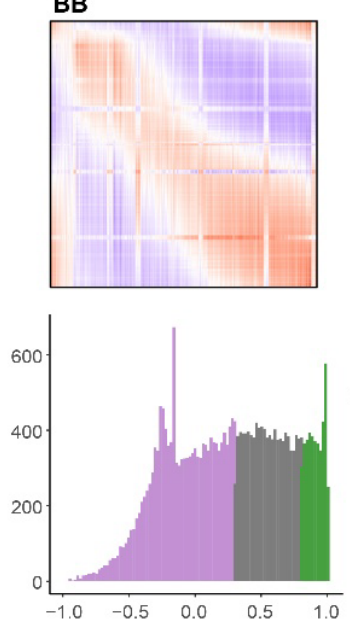

DD
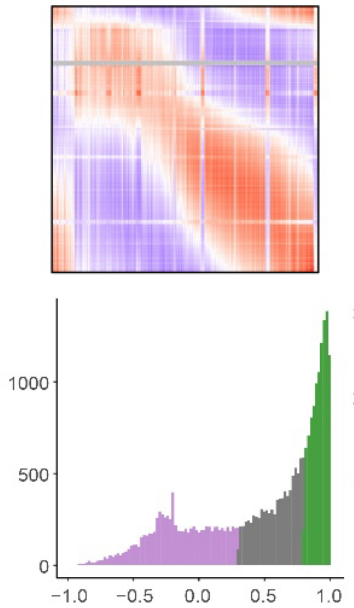

c

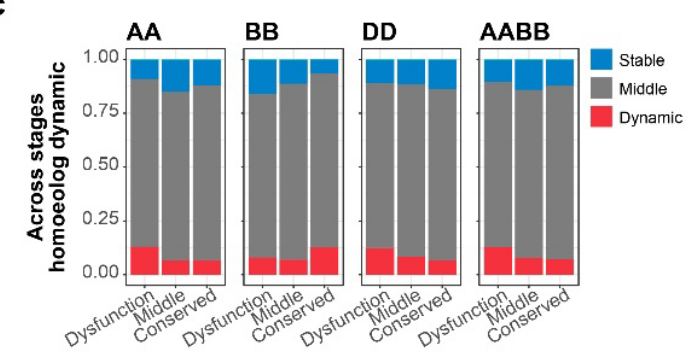

AABB
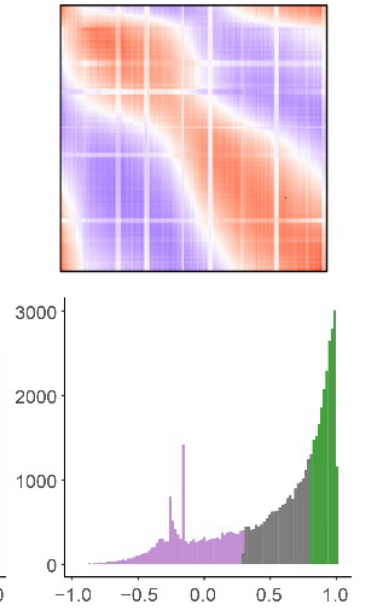

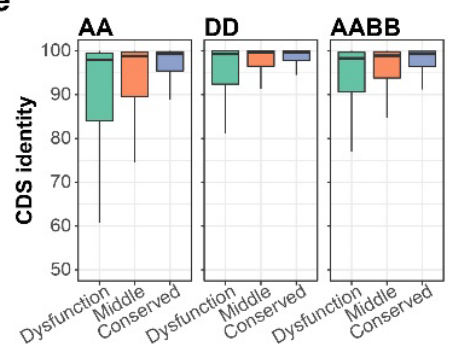

f

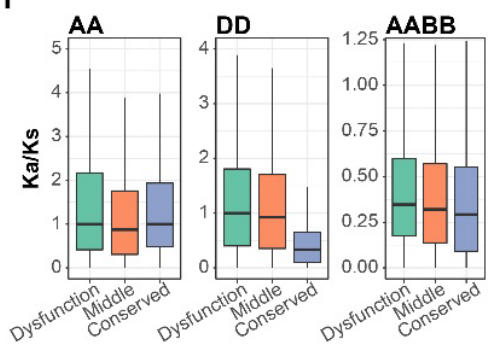

h

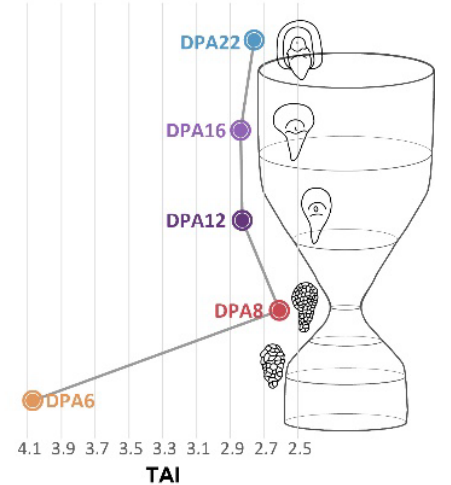

j

i ATAC

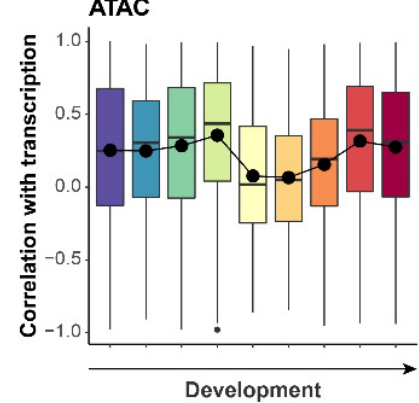

H3K4me3

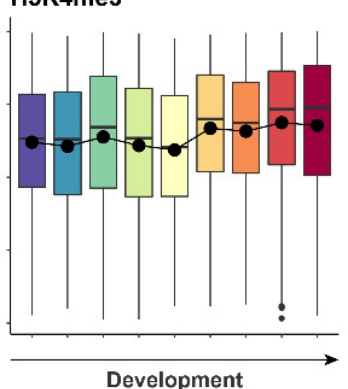

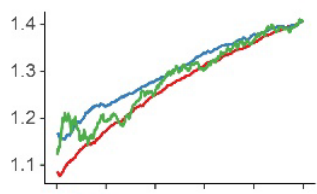

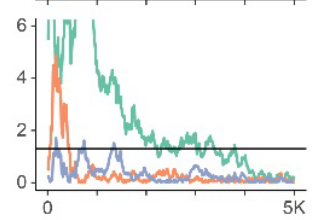

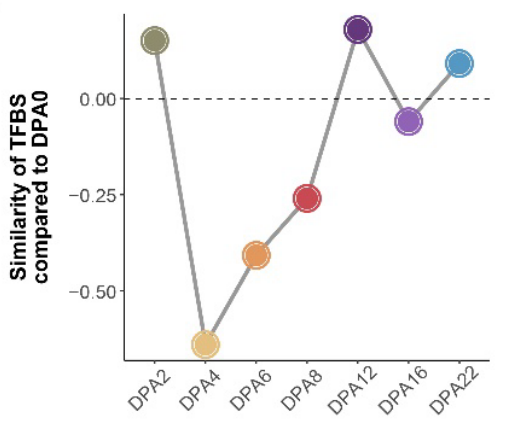


Fig. S9 | Comparisons of embryogenesis among different ploidy wheat and hourglass model a, Comparisons of gene expressions between hexaploid wheat (AABBDD) and ancestors (AA, BB, $\mathrm{DD}$ and $\mathrm{AABBDD}$ ) and corresponding Pearson correlation index. Each row represents an individual gene in hexaploid wheat and each line represents an individual gene in ancestor. Pearson correlation index was from -1 to 1 , and indicated by colors from blue to red. Genes were clustered into three categories based on Pearson index from the comparisons between hexaploid wheat and ancestors, which are dysfunction, middle and conserved (bottom).

b, c, subgenome synteny (b) and triad dynamic expression across developmental stages (c) for gene sets defined in $\mathbf{a}$.

d, Percentage of genes has paralogous in corresponding ancestors for gene sets defined in $\mathbf{a}$..

e, Sequence similarity between hexaploid wheat and different ancestors for the corresponding gene sets defined in $\mathbf{a}$.

f, $\mathrm{Ka} / \mathrm{Ks}$ comparisons for different gene sets defined in $\mathbf{a}$.

g, TEs insertion frequency at different positions relative to different gene sets (top) and comparisons between different gene sets (bottom). Mann-Whitney U test (two-sided) was used for the significant difference.

h, TAI index for expressed genes from DPA6 to DPA22, which represent an hourglass model.

i, ATAC but not $\mathrm{H} 3 \mathrm{~K} 4 \mathrm{me} 3$ contributed to genes expression across wheat embryogenesis.

j, Similarity of TFs binding pattern compared with that in DPA0. All TFs binding patterns were calculated by chromVAR. 
a

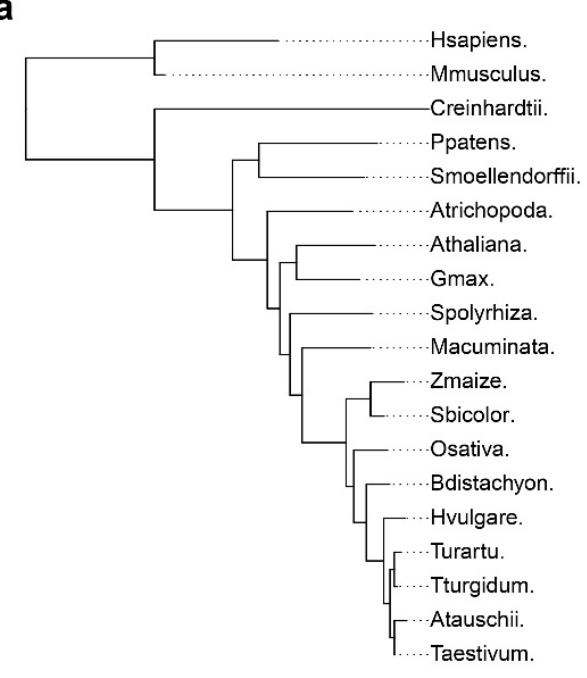

d

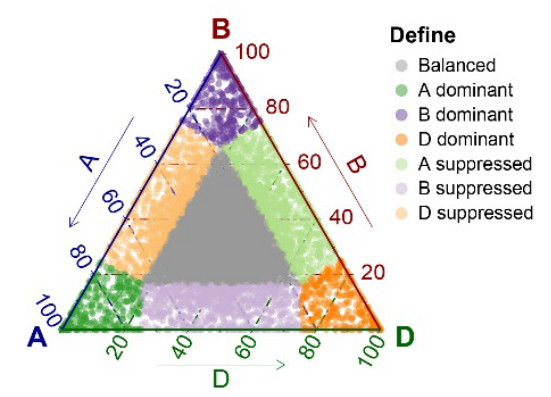

b

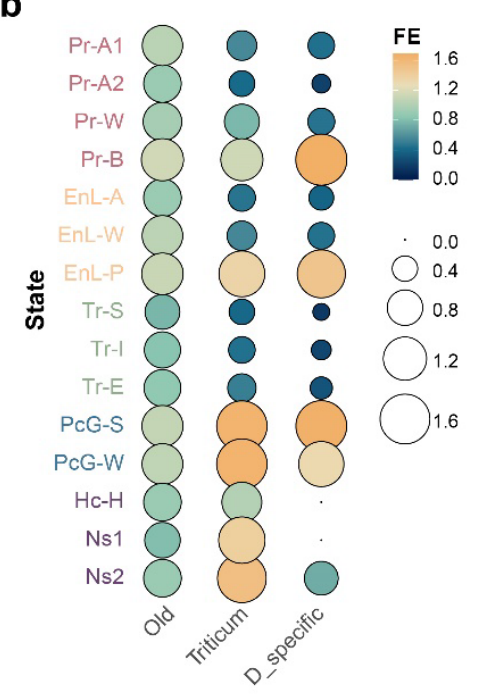

C
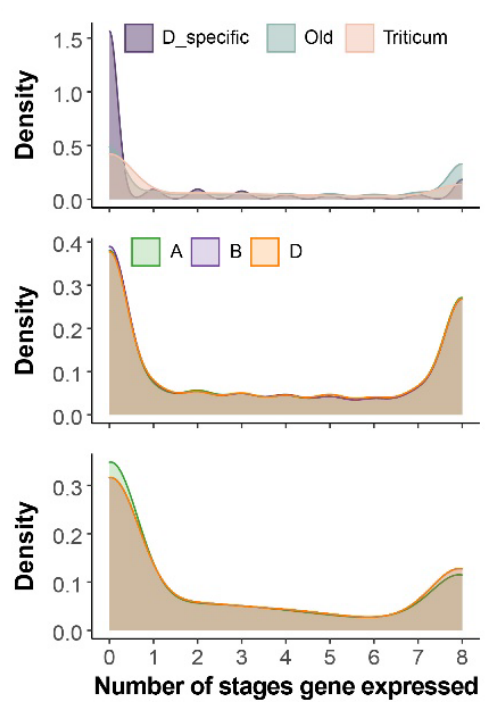

e

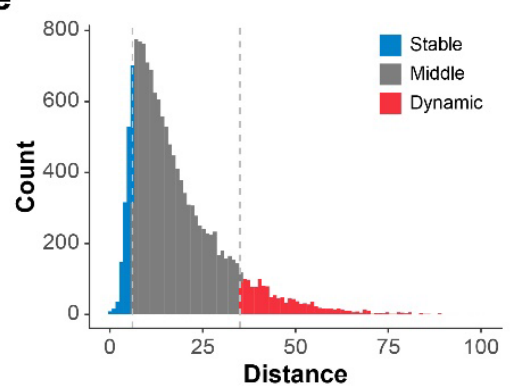

f

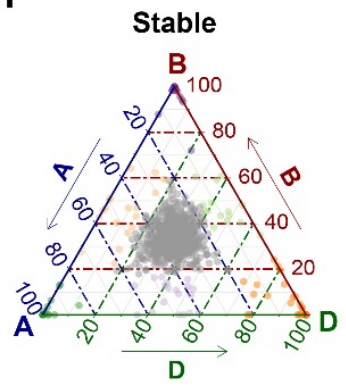

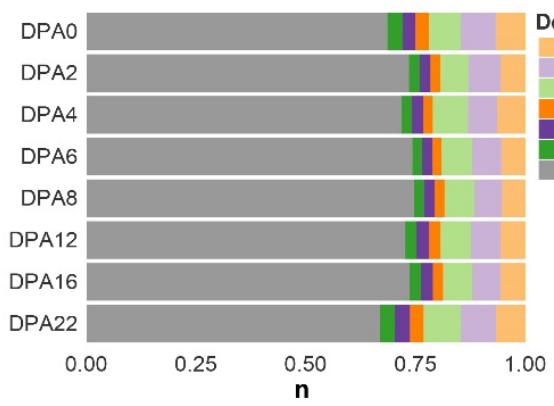

Define

D suppressed
B suppressed A suppressed dominant A dominant A dominant
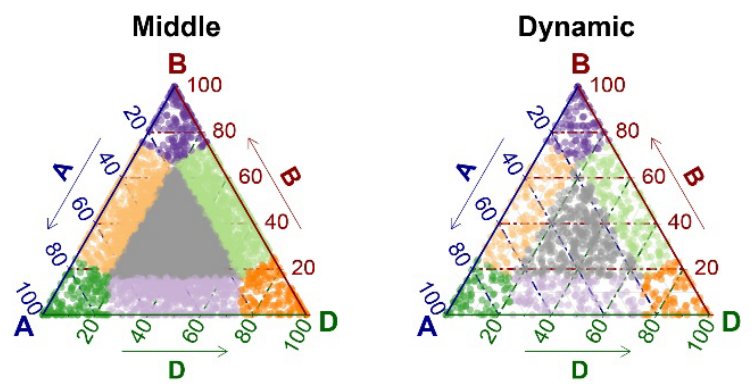

Fig. S10 | Chromatin landscape affected sub-genome bias expression

a, Phylostratigraphic map of various wheat species

b. Chromatin states enrichment for different evolutionary age genes in wheat embryo.

c, Expression spectrum of different evolutionary age genes in wheat embryo.

d, Define of homeologs bias expression (left) and percentage of different homeologs bias expression types across eight wheat embryonic developmental stages (right).

e, Homeologs dynamic expression across wheat embryogenesis. Euclidean distance method was used to analysis as (Ramírez-González et al., 2018).

f, Homeologs bias expression for gene sets defined in e. 
bioRxiv preprint dol: https://doi.org/10.1101/2022.01.21.477188; this version posted January 23, 2022. The copyright holder for this preprint (which was not certified by peer review) is the author/funder. All rights reserved. No reuse allowed without permission.

Balanced

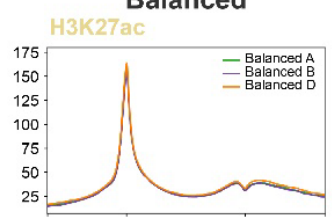

H3K9ac

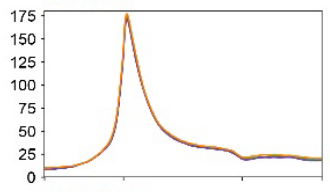

$\mathrm{H} 3 \mathrm{~K} 4 \mathrm{me} 3$

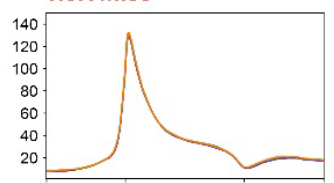

H3K36me3

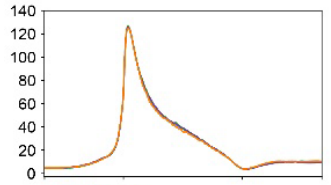

H3K4me1

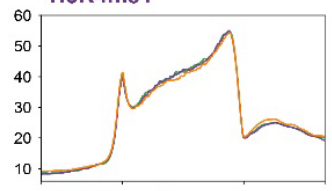

H3K27me3

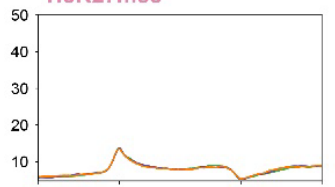

H3K9me2

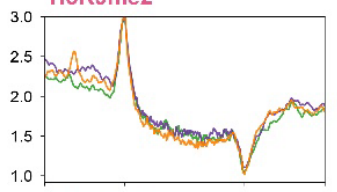

H3K9me3

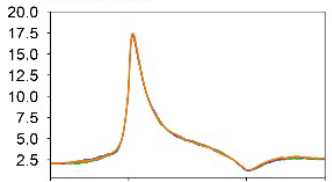

Pol II

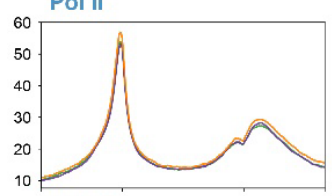

120 H2A.Z

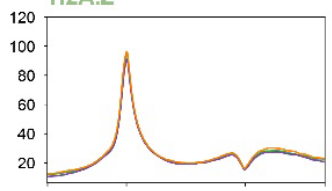

ATC

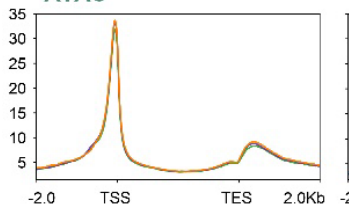

Suppressed
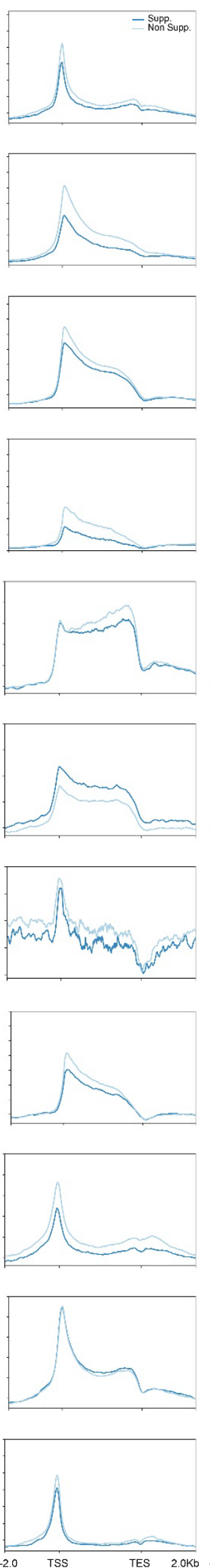

Dominant
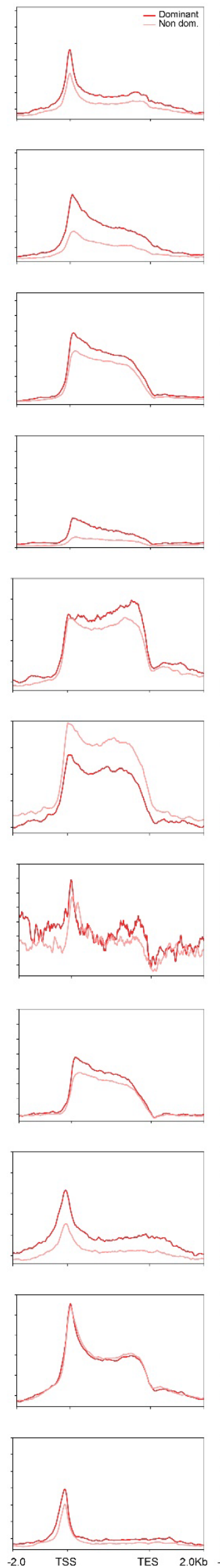

Combined
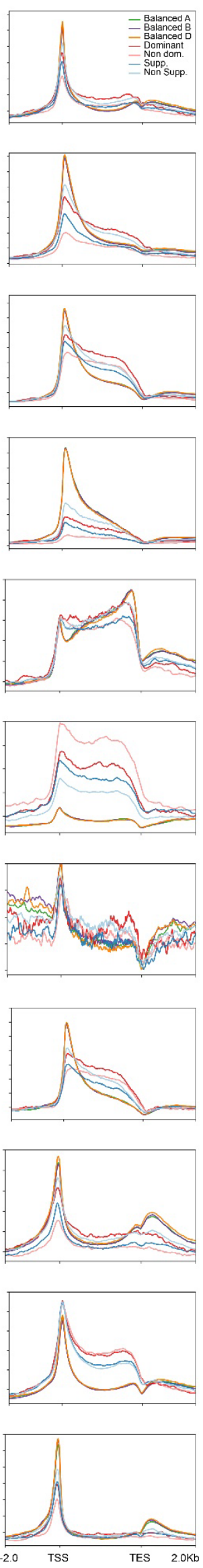
Fig. S11 | Epigenetic modification on different types of homeologs bias expressed genes.

Balanced, suppressed, and dominant genes were shown in the first three columns, and a combination of different types of homeologs was shown in the fourth column. Epigenetic modification signals were normalized using RPKM, with 10 bp bin size. 
a

\section{TaFIE}

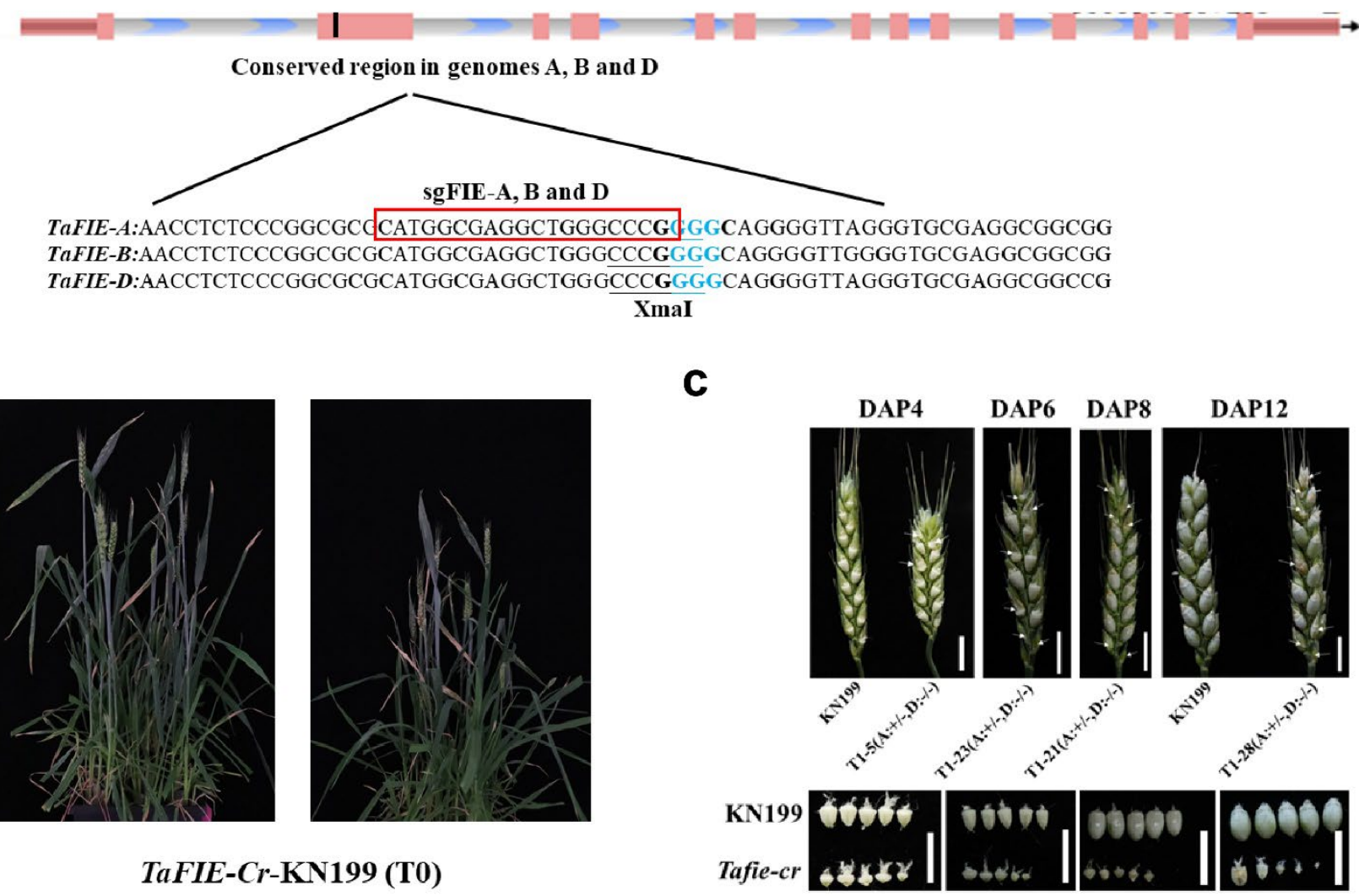

Fig. S12 | Loss of core component of PRC2 led to aberrant seeds in wheat.

a, Genotyping of CRISPR plant at TaFIE.

b, Growth phenotype of TaFIE-Cr-KN199 as compared to wildtype KN199.

c, Aberrant seed produced by TaFIE-Cr-KN199. 